\title{
Platinum(IV)-Ferrocene Conjugates and their Cyclodextrin Host-Guest Complexes
}

Grégory Thiabaud, Louis Harden-Bull, Yoo-Jin Ghang, Sajal Sen, Xiaodong Chi, James L. Bachman, Vincent M. Lynch, Zahid H. Siddik, Jonathan L. Sessler*

1. General procedures

2. Synthesis and characterizations of compounds $1,2,3$, and complexes $2 @ \beta-C D$, $\beta-C D-S i R$, and 2@ $\beta-C D-S i R$ 


\section{General procedures:}

Starting materials were purchased from Fisher Scientific, Sigma Aldrich, Ark Pharm. Inc (oxaliplatin), or TCl (cyclodextrins) and used without further purification unless otherwise specified. Solvents were purified using a solvent purifier system (Vacuum Atmospheres). Dichloromethane was freshly distilled after being dried over $\mathrm{CaH}_{2}$ under argon. Reaction progress was monitored by thin-layer chromatography (TLC silica gel 60 $F_{254}$, Silicycle UltraPure silica gels). Analytical and semi-preparative RP-HPLC were performed on a Thermo scientific Dionex Ultimate 3000 instrument equipped with PDA detector (set at $254 \mathrm{~nm}$ for Pt compounds). The analytical column was a Syncronis C18 column, $5 \mu \mathrm{m}, 4.6$ x $250 \mathrm{~mm}$ (Thermo Scientific); the mobile phase was made of an increasing gradient (from $10 \%$ to $99 \%$ in $10 \mathrm{~min}$ ) of acetonitrile into water, both containing $0.1 \%$ acetic acid. In this case the flow rate was set to $1.2 \mathrm{ml} / \mathrm{min}$. The semi-preparative column was a Hypersil gold C18, $5 \mu \mathrm{m}, 10 \times 150 \mathrm{~mm}$ (Thermo Scientific). The gradient was the same that for the analytical studies; however, a flow rate of $5 \mathrm{ml} / \mathrm{min}$ was used. Mass spectrometric analyses were carried out in The University of Texas at Austin (UT Austin) Mass Spectrometry Facility. Low- and highresolution electro- spray mass spectrometric (ESI-MS) analyses were carried out using Thermo Finnigan LTQ and Qq-FTICR (7 Tesla) instruments, respectively. ${ }^{1} \mathrm{H}$ NMR spectra were recorded using a Varian 400 $\mathrm{MHz}$ instrument unless noted otherwise. 2D correlation experiments were recorded using a Bruker AVIII HD 500 equipped with a Prodigy liquid nitrogen cryoprobe. ROESY analyses were carried out on a Varian $600 \mathrm{MHz}$ instrument (relaxation delay: $2 \mathrm{sec}$; flip angle $45^{\circ}$; line broadening $200 \mathrm{~Hz} ; 3500$ ppm wide). UVvis spectra were recorded on a Varian 5000 UV-vis-NIR spectrophotometer; LC-MS analysis were carried out using a Agilent 6120 Quadrupole instrument, starting the elution at $5 \% \mathrm{MeOH}$ (with $0.1 \%$ formic acid) in water (with $0.1 \%$ formic acid) increasing to $100 \% \mathrm{MeOH}$ over the course of 12 minutes. Isothermal calorimetry experiments were carried out on a VP-ITC MicroCal microcalorimeter; SEM experiments were carried out on a HITACHI S-5500 SEM/STEM instrument. ICP-MS measurements of intracellular Pt levels were carried out on an Agilent 7500ce ICP-MS in the Jackson School of Geosciences at UT Austin.

\section{Synthesis and characterizations of compounds 1,2 , and 3 and complexes $2 @ \beta-C D, \beta-C D-S i R$, and $2 @ \beta-C D-S i R$}

Compound 1 was synthesized by adopting the procedure described in references [S1a] and [S1b]. Briefly, $3 \mathrm{ml}$ of hydrogen peroxide were added to $1 \mathrm{~g}$ of oxaliplatin as a suspension in $12 \mathrm{ml}$ of water. The mixture was stirred in the dark for 24 hours at room temperature, followed by $3 \mathrm{~h}$ at $50^{\circ} \mathrm{C}$. The solution was cooled in a refrigerator and filtered through membrane filters (ATTP, $0.8 \mu \mathrm{m}$ pore, $0,47 \mathrm{~mm}$ diameter) then washed with water. This first operation yields the oxidized version of oxaliplatin with two hydroxyl group as axial ligands: $\mathrm{Pt}^{4+}(\mathrm{OH})_{2}$. After being dried under vacuum, the resulting white/yellowish powder was dissolved in $6 \mathrm{ml}$ of anhydrous dimethylformamide (DMF) to which 3 equiv of succinic anhydride were added. The mixture was heated to $50^{\circ} \mathrm{C}$ in the dark for 24 hours. After checking by HPLC that the conversion was complete within the limits of analysis, the DMF was removed under vacuum (at $50^{\circ} \mathrm{C}$ ). $\mathrm{MeOH}$ was added to the solid residue. The mixture was then sonicated and then filtrated using a PTFE syringe filter $(0.2 \mu \mathrm{m}$ pore size). The solvent was concentrated under vacuum, before acetone and ether were added. The resulting white precipitate was collected by filtration through a Nylon membrane disc ( $47 \mathrm{~mm}$ diameter, 0.45 $\mu \mathrm{m}$ pore size). After drying, prodrug 1 was obtained as a pale brown powder in about $80 \%$ yield. It was used without any further purification. 
Compound 11: Prodrug 1 (68 mg, $11 \mu \mathrm{mol}$ ) as a solution in anhydrous dimethylformamide (DMF) was reacted with benzotriazol-1-yl-oxytripyrrolidinophosphonium hexafluorophosphate PyBOP (2.7 equiv) and diisopropylethylamine (2.7 equiv) in the dark for $2 \mathrm{~h}$ at room temperature. Thereafter, L5 (2.1 equiv) (synthesized following the protocol described in ref. [S2]) was added and the reaction was left for $15 \mathrm{~h}$. The bulk of the DMF was then removed under reduced pressure and the product 11 was purified by column chromatography. The elution was carried out using an increasing amount of methanol in DCM (from 2 to $15 \%)$.

Compound 2: Prodrug 1 (68 mg, $11 \mu \mathrm{mol}$ ) as a solution in anhydrous dimethylformamide (DMF) was reacted with benzotriazol-1-yl-oxytripyrrolidinophosphonium hexafluorophosphate PyBOP (2.7 equiv) and diisopropylethylamine (2.7 equiv) in the dark for $2 \mathrm{~h}$ at room temperature. Thereafter, L3 (2.1 equiv) (synthesized following the protocol described in ref. [S2]) was added and the reaction was left for $15 \mathrm{~h}$. The bulk of the DMF was then removed under reduced pressure and the product 2 was purified by column chromatography (silica gel, $\mathrm{R}_{\mathrm{f}}=0.5$ with $15 \% \mathrm{MeOH}$ in dichloromethane (DCM)). The elution was carried out using an increasing amount of methanol in DCM (from 2 to 15\%). After removal of the solvent under reduced pressure, target 2 was obtained as an orange powder $(60 \%)$. Elemental analysis for $\mathrm{C}, \mathrm{H}, \mathrm{N}$ : theoretical: 44.26, 4.60, 7.37; found: 44.06, 5.19, 7.64. ${ }^{195} \mathrm{Pt}$ NMR ( $d_{6}$-DMSO): $1600.8 \mathrm{ppm}$.

\section{Target Compound Screening Report}

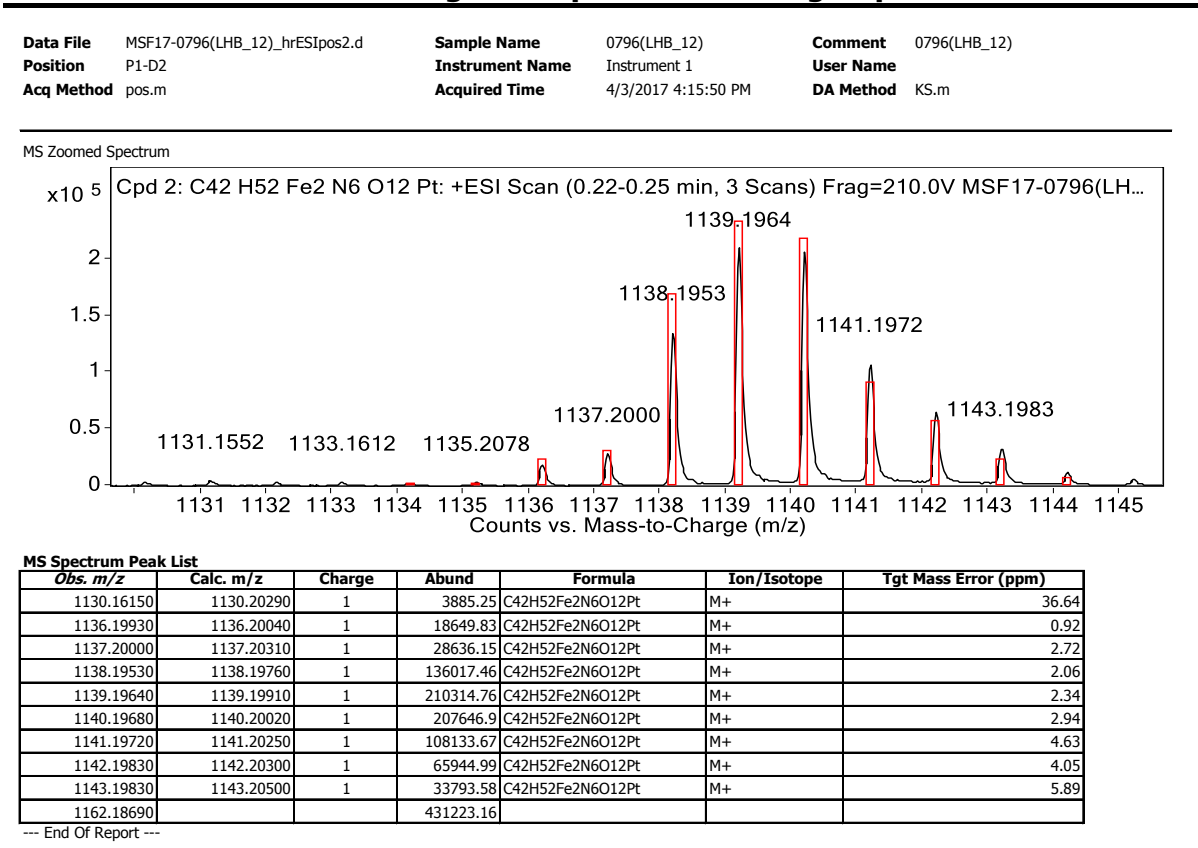

Figure S1: High-resolution mass spectrum (electrospray ionization method) showing the isotopic pattern and error relative to what is predicted for $\left[2+\mathrm{H}^{+}\right]^{+}$. 


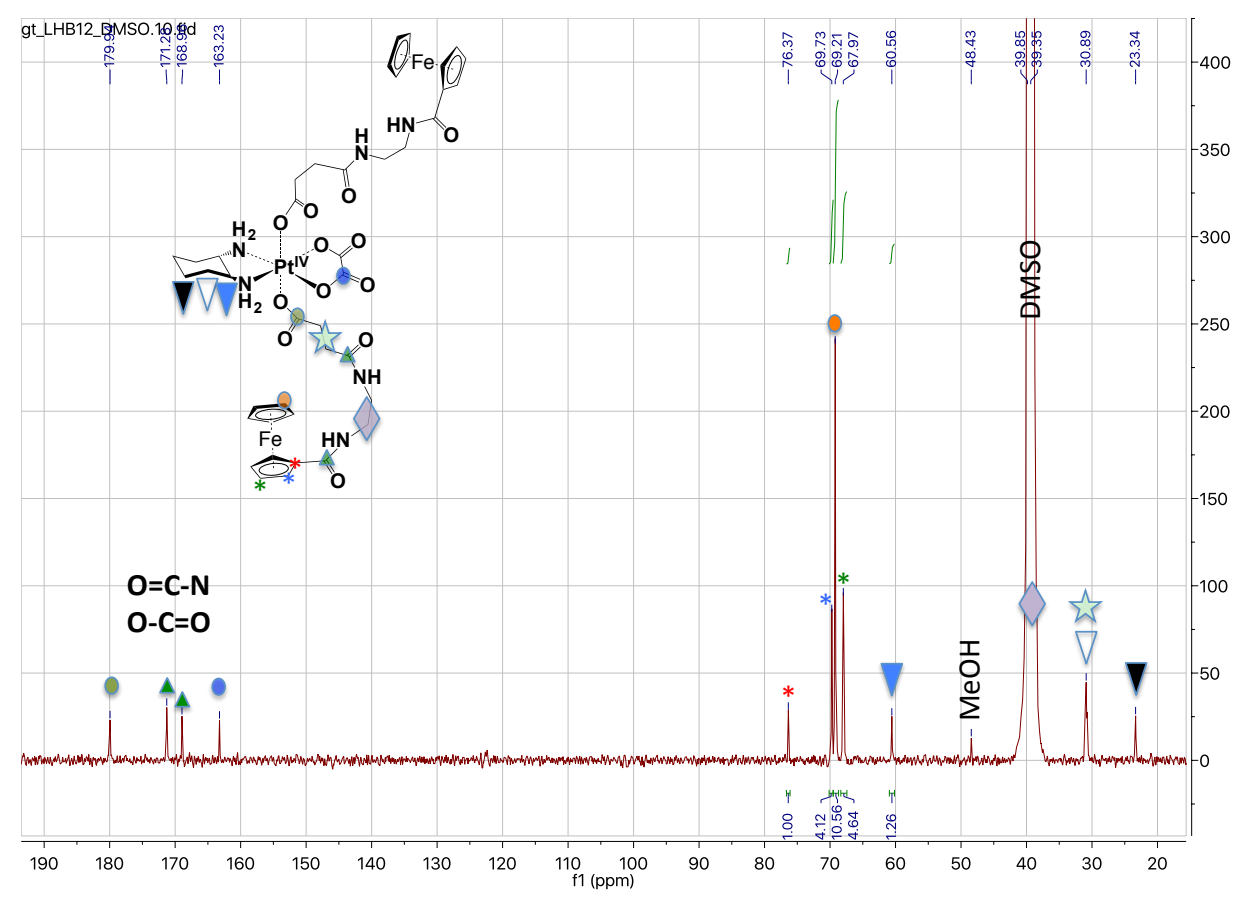

Figure S2: ${ }^{13} \mathrm{C}$ NMR spectrum ( $d_{6}-\mathrm{DMSO}, 300 \mathrm{~K}$ ) of 2 . Note: ethylenediamine $\mathrm{CH}_{2}$ protons are hidden by the DMSO peak.

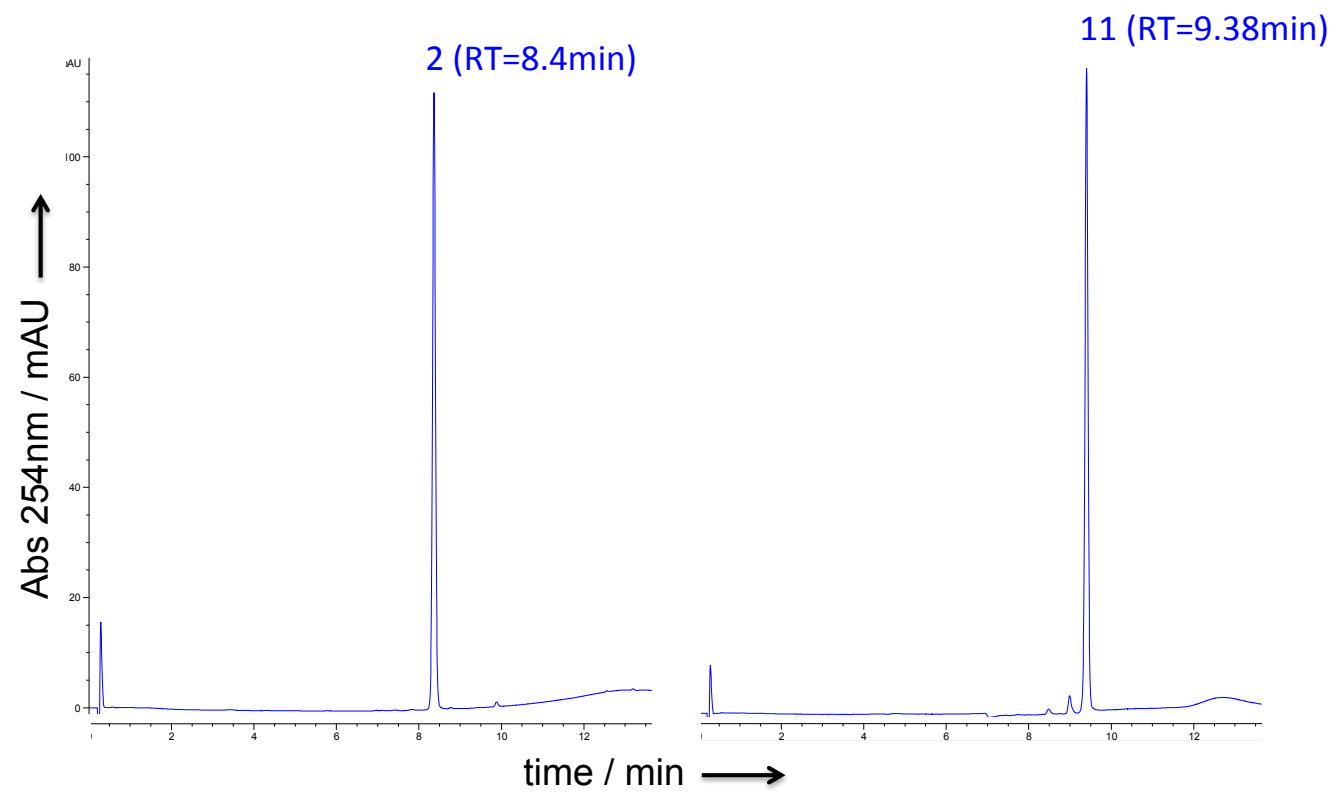

Figure S3: Chromatograms (LC-MS) of 2 (left) and 11 (right) obtained with the detector set at $254 \mathrm{~nm}$. Note the significant difference in the retention time for these two ostensibly similar analogues. 


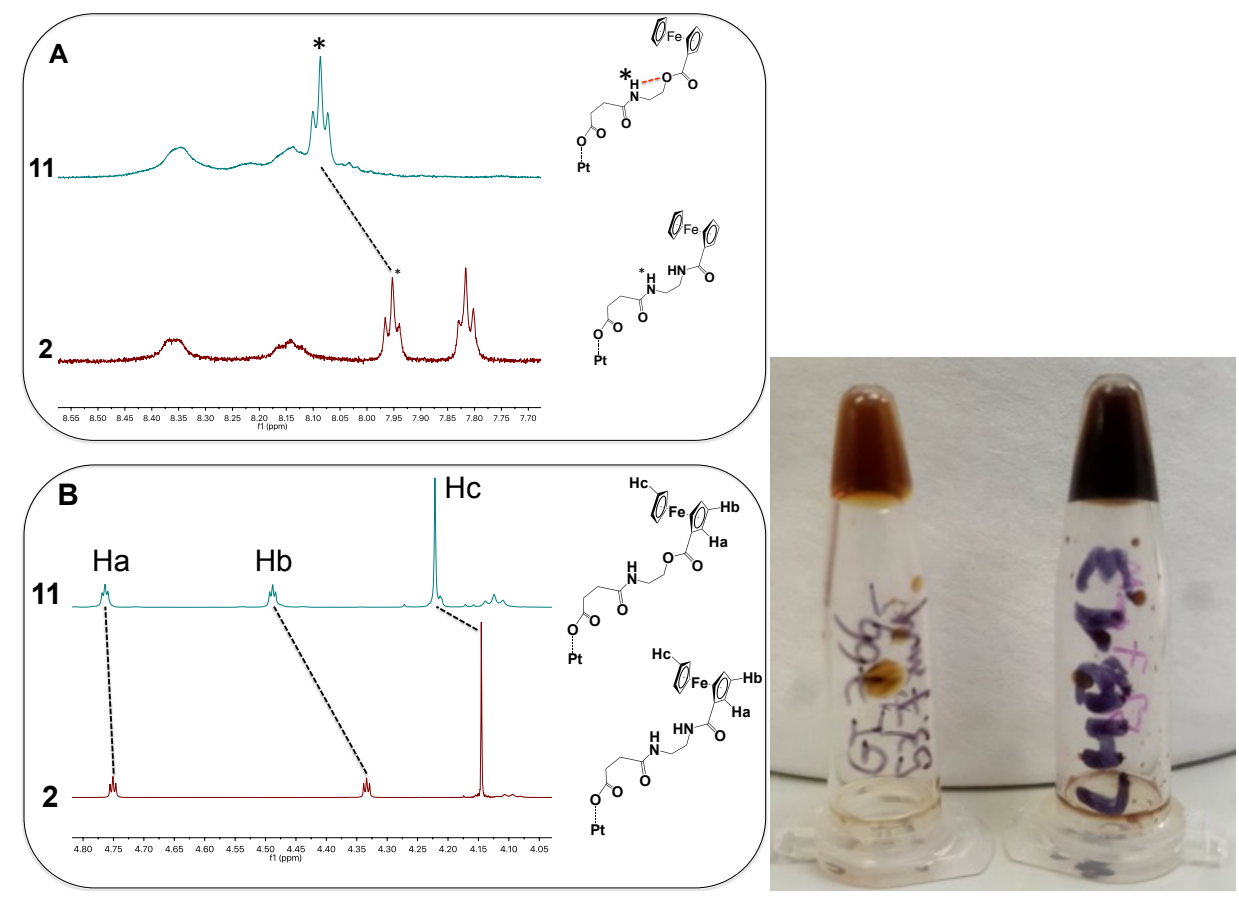

Figure S4: Partial ${ }^{1} \mathrm{H}$ NMR spectra ( $400 \mathrm{MHz}, 300 \mathrm{~K}, d_{6}$-DMSO) of 2 and 11.

A: Lower field region showing the $\mathrm{NH}$ signals, the values for which are thought to reflect differences in the extent of intramolecular hydrogen bonding. B: Signals corresponding to the protons on the ferrocene moiety. The picture on the right shows solutions of 2 (left) and 11 (right) in DMSO after $5 \mathrm{~h}$ at room temperature. Initially, the solution containing $\mathbf{1 1}$ displayed the same color as $\mathbf{2}$.

Compound 3: Prodrug 1 (60 mg, $95 \mu \mathrm{mol})$ as a solution in anhydrous DMF $(5 \mathrm{ml})$ was reacted with PyBOP (2.7 equiv) and diisopropyethylamine (2.7 equiv) in the dark for $2 \mathrm{~h}$ at room temperature. Thereafter L3 (1 equiv), synthesized following the protocol described in the ref. [S1], was added dropwise. This was followed by the addition of 3-amine- $\beta$-cyclodextrin (1 equiv). The reaction was then left for $15 \mathrm{~h}$. The bulk of the DMF was then removed under reduced pressure and the crude material was purified on reverse phase high performance liquid chromatography (RP-HPLC). The fractions containing 3 were combined and lyophilized to give 3 as a fluffy orange solid ( $20 \mathrm{mg}, 10 \%)$. 


\section{Target Compound Screening Report}

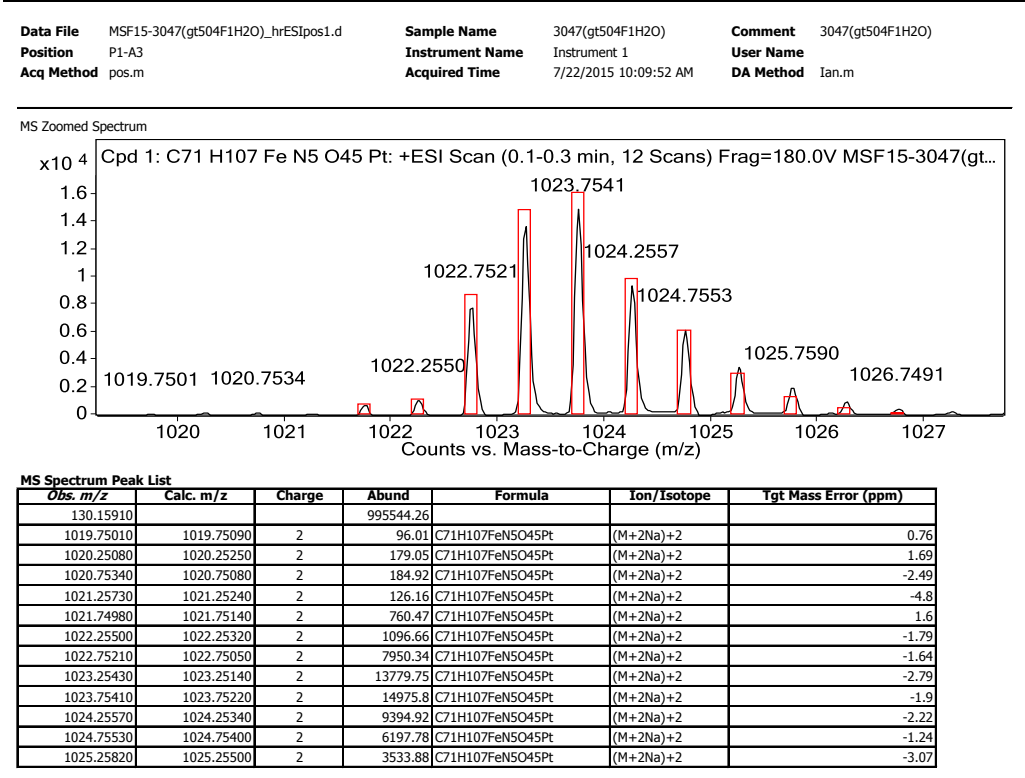

Figure S5: High-resolution mass spectrum (electrospray ionization method) showing the isotopic pattern and error relative to what is predicted for $\left[3+2 \mathrm{Na}^{+}\right]^{2+}$.

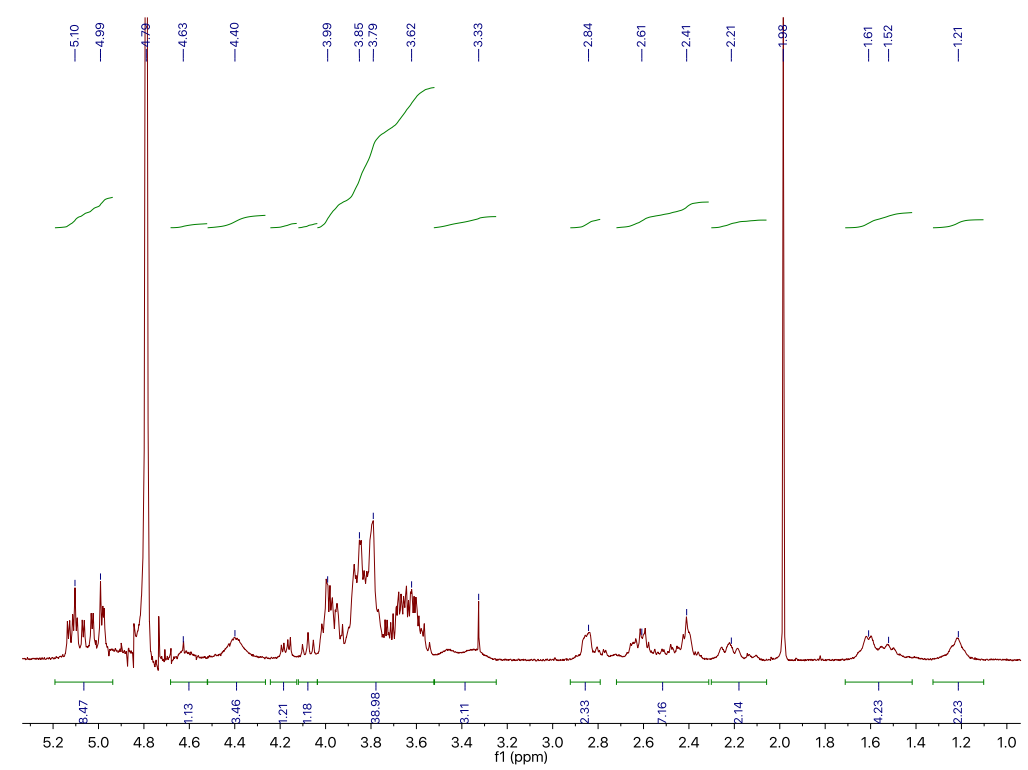

Figure S6: Partial ${ }^{1} \mathrm{H}$ NMR $(400 \mathrm{MHz}, 300 \mathrm{~K})$ spectrum of the hybrid compound 3 recorded in $\mathrm{D}_{2} \mathrm{O}$. 


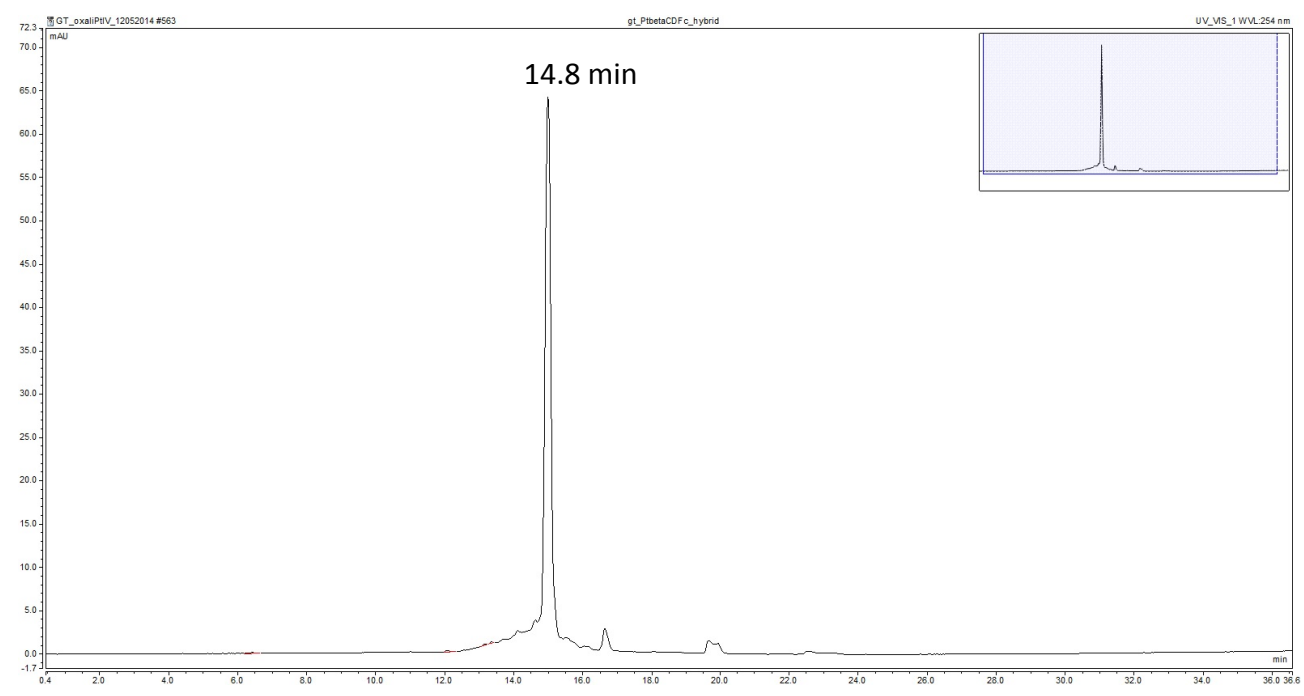

Figure S7: RP-HPLC chromatogram (detector set at $254 \mathrm{~nm}$ ) of 3.

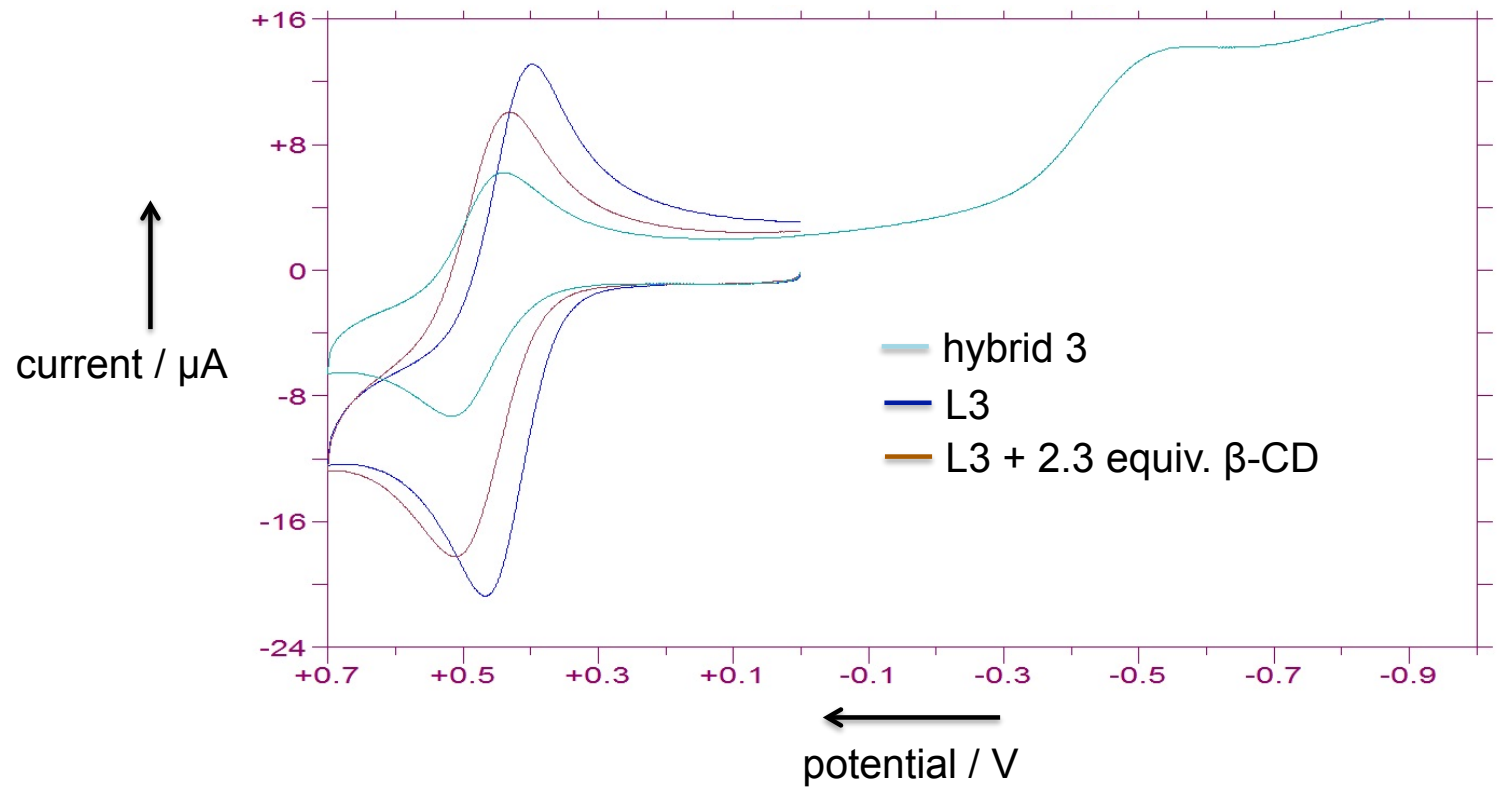

Figure S8: Cyclic voltammograms (PBS, $\mathrm{KCl} 0.1 \mathrm{M}, 100 \mathrm{mV} / \mathrm{sec}$, reference electrode: $\mathrm{Ag} / \mathrm{AgCl}$; working electrode: glassy carbon; auxiliary electrode: Pt) of $\mathbf{3}(1.25 \mathrm{mM})$ compared to those of $\mathbf{L} \mathbf{3}$ determined in the absence and presence of $\beta-\mathrm{CD}$. The redox potential for the $\mathrm{Fc}^{+} / \mathrm{Fc}$ in $\mathbf{3}$ is similar to the one for $\mathbf{L} \mathbf{3}$ in the presence of $\beta-C D$. 
Scanning electron microscopic studies of $\mathbf{3}$

A drop of a solution of $\mathbf{3}$ in water (mM concentration) was placed onto a microscope glass slide and the solvent was removed under a gentle nitrogen flow. The sample was frozen and then dried to obtain a powder that was then placed onto a carbon double-sided conductive tape prior to carrying out the SEM analysis.
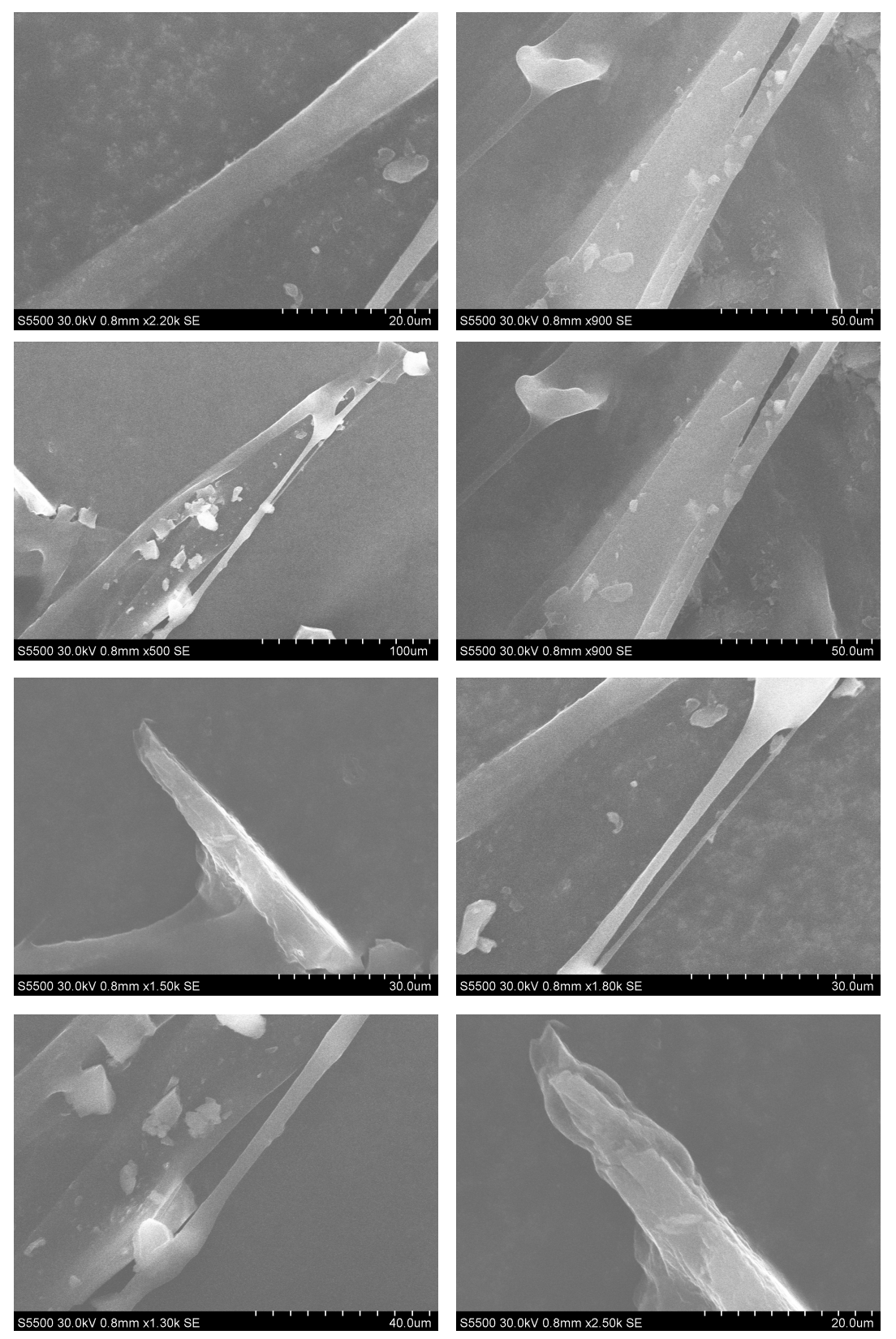

Figure S9: Scanning electron micrographs of $\mathbf{3}$ (solid residue formed upon evaporation of an aqueous solution). 
Complex 2@ $\beta-C D$ : Host-guest interactions of 2 with $\beta$-cyclodextrin (and (2-Hydroxypropyl)- $\beta$ cyclodextrin $=$ HP- $\beta-C D$ )

Synthesis of $2 @ \beta-C D$ : Complex 2 was added as a solid to an aqueous solution of $\beta-C D$ or HP- $\beta-C D$. The solution was sonicated and vortexed for about 5 min until the solid was totally dissolved. The solution was then filtered and used without further purification.
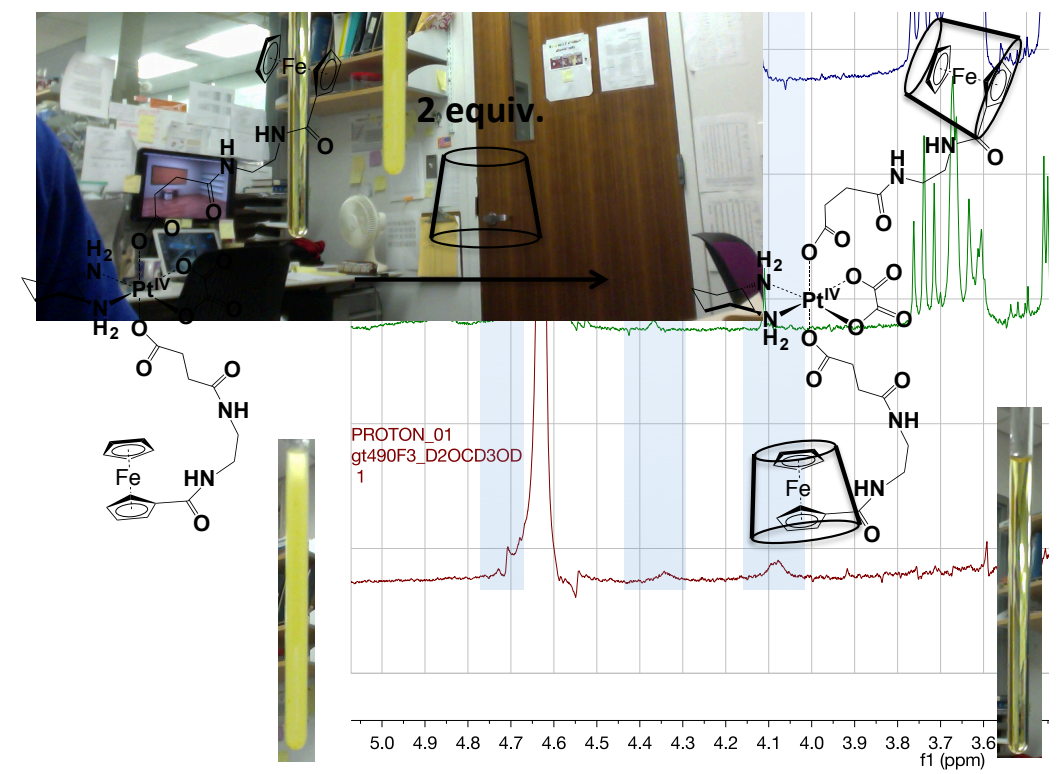

Figure S10: Solubilization of 2 in water observed upon the addition of $\beta$-cyclodextrin (or HP- $\beta$ cyclodextrin). The two pictures are photographs of NMR tubes taken before (turbid) and after addition of $\beta$-cyclodextrin (fully transparent).

Note: both $\beta$-cyclodextrin and HP- $\beta$-cyclodextrin were tested and gave similar results in term of solubilization of 2. 


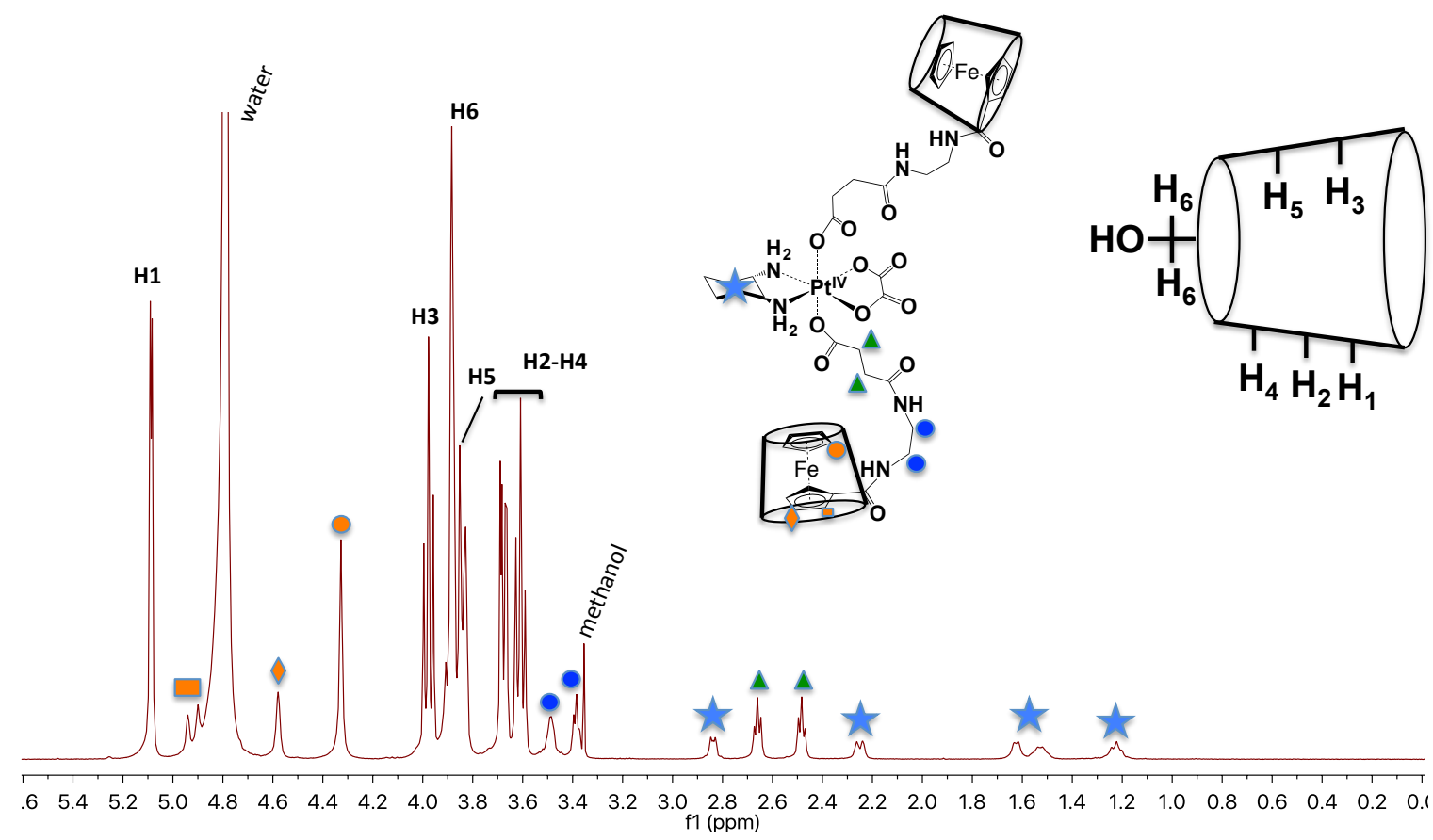

Figure S11: Partial ${ }^{1} \mathrm{H}$ NMR $\left(500 \mathrm{MHz}, 300 \mathrm{~K}\right.$, cryoprobe) spectrum of $2 @ \beta-C D$ recorded in $\mathrm{D}_{2} \mathrm{O}$.
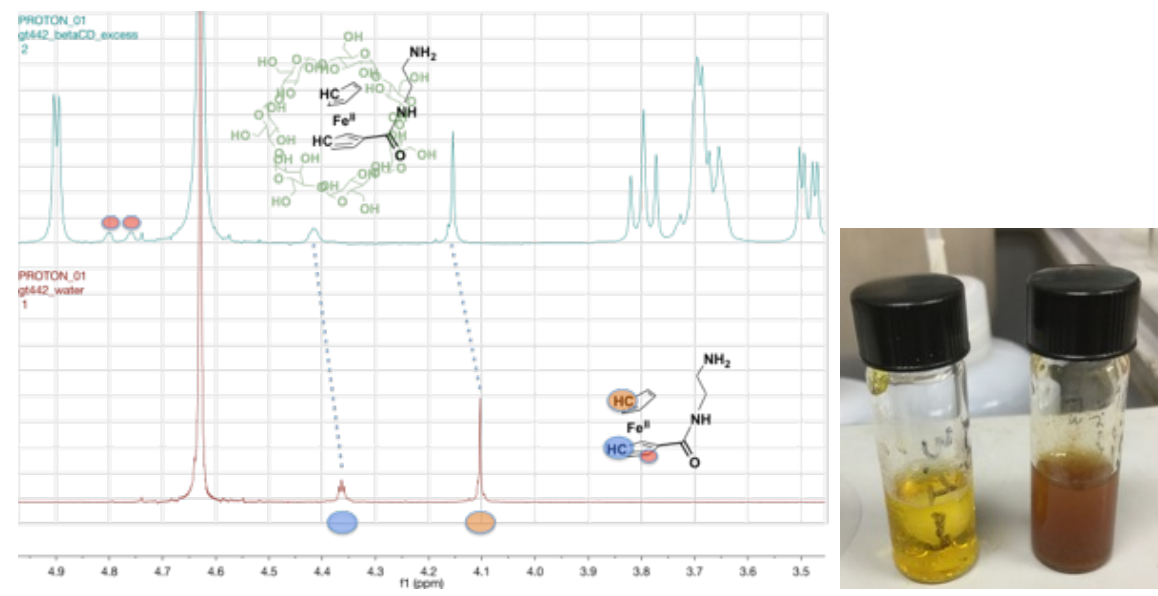

Figure S12: Partial ${ }^{1} \mathrm{H}$ NMR (400 MHz, $300 \mathrm{~K}, \mathrm{D}_{2} \mathrm{O}$ ) spectra of L3 recorded before (bottom) and after (top) addition of $\beta-C D$ (excess). The picture on the right depicts water solution of L3 kept for several days at $20^{\circ} \mathrm{C}$ (left) or at room temperature $22^{\circ} \mathrm{C}$ (right). 


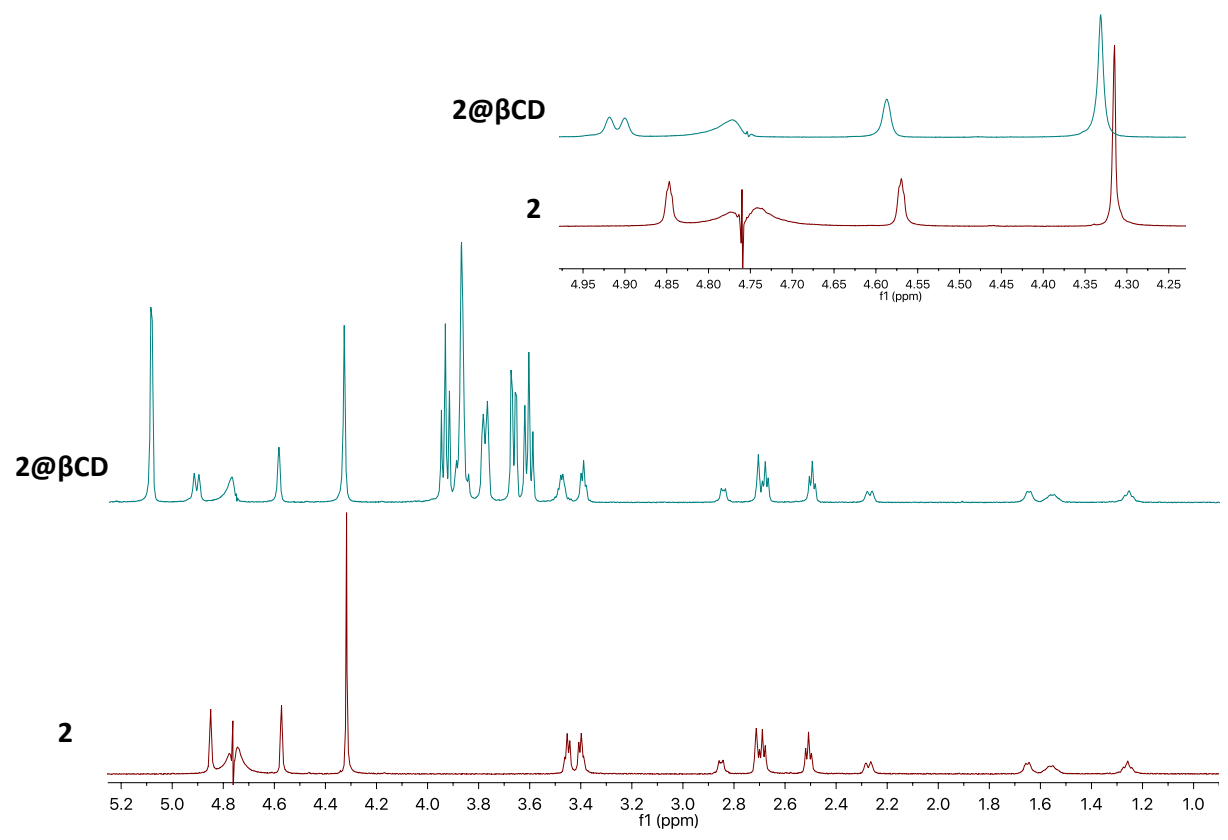

Figure S13: ${ }^{1} \mathrm{H}$ NMR $\left(600 \mathrm{MHz}, 300 \mathrm{~K}, 30 \% d_{6}\right.$-DMSO in $\left.\mathrm{D}_{2} \mathrm{O}\right)$ spectra of $2(3.9 \mathrm{mM})$ recorded before and after the addition of $\beta-C D$ ( 2 equiv) with water peak suppression (pre-saturation method). The inlet shows a portion of the spectrum so as to facilitate visualization of the Fc peaks.
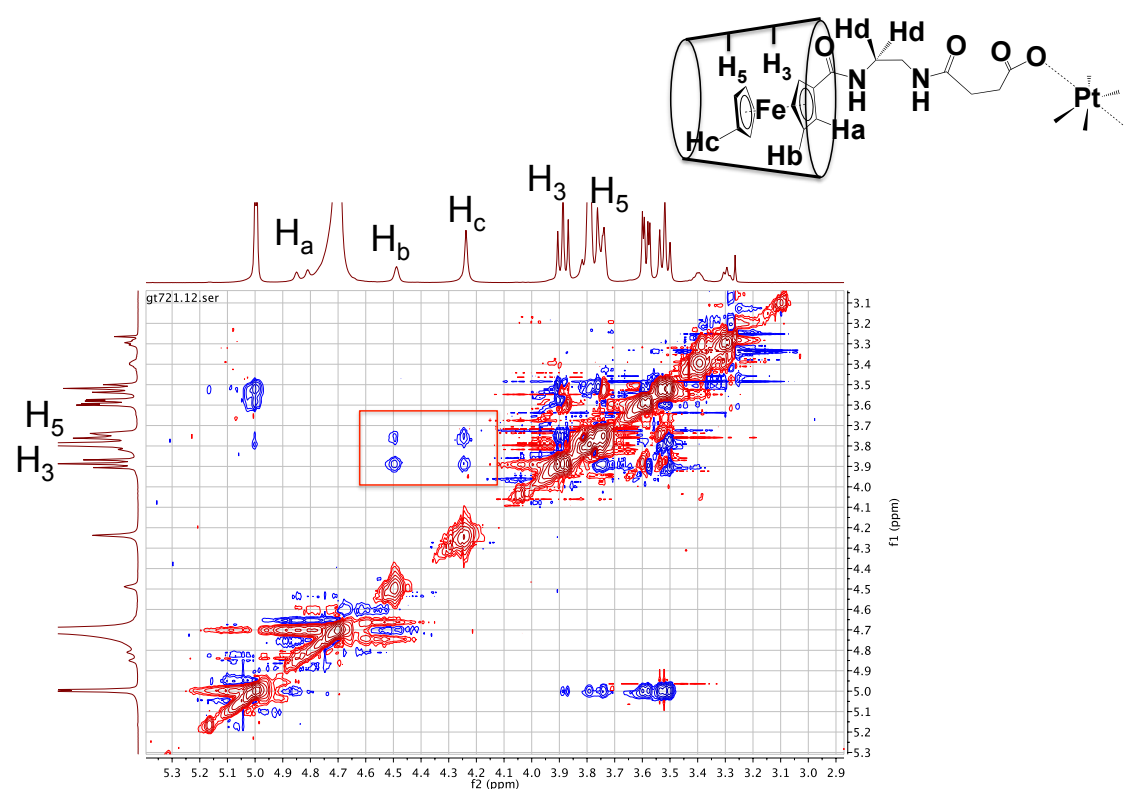

Figure S14: ${ }^{1} \mathrm{H}$ NMR ROESY experiment involving a sample of $2(3.9 \mathrm{mM})+2$ equiv $\beta$-CD $\left(30 \% d_{6}\right.$-DMSO in $\mathrm{D}_{2} \mathrm{O}, 500 \mathrm{MHz}, 300 \mathrm{~K}$ ). The red square highlights the weak correlation between protons on the ferrocene moiety $\left(\mathrm{H}_{\mathrm{b}} / \mathrm{H}_{\mathrm{c}}\right)$ and those on the $\beta$-CD receptor $\left(\mathrm{H}_{3} / \mathrm{H}_{5}\right)$. 

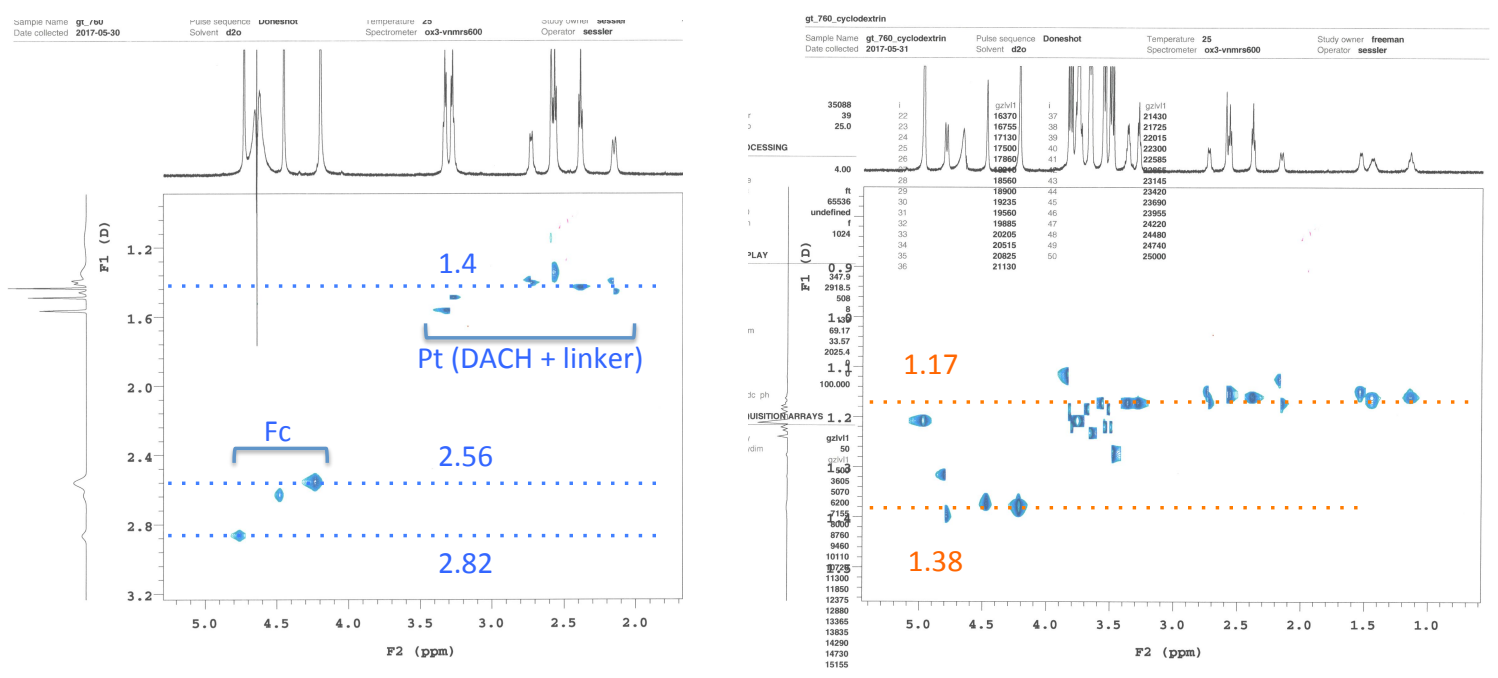

Figure S15: ${ }^{1} \mathrm{H}$ NMR spectral study $\left(30 \% d_{6}\right.$-DMSO in $\left.\mathrm{D}_{2} \mathrm{O}, 600 \mathrm{MHz}, 300 \mathrm{~K}\right)$ showing the results of DOSY experiments involving $2(3.9 \mathrm{mM})$ before (left) and after addition (right) of $\beta-C D$ (2 equiv). Note: The water peak was suppressed using the pre-saturation method. 

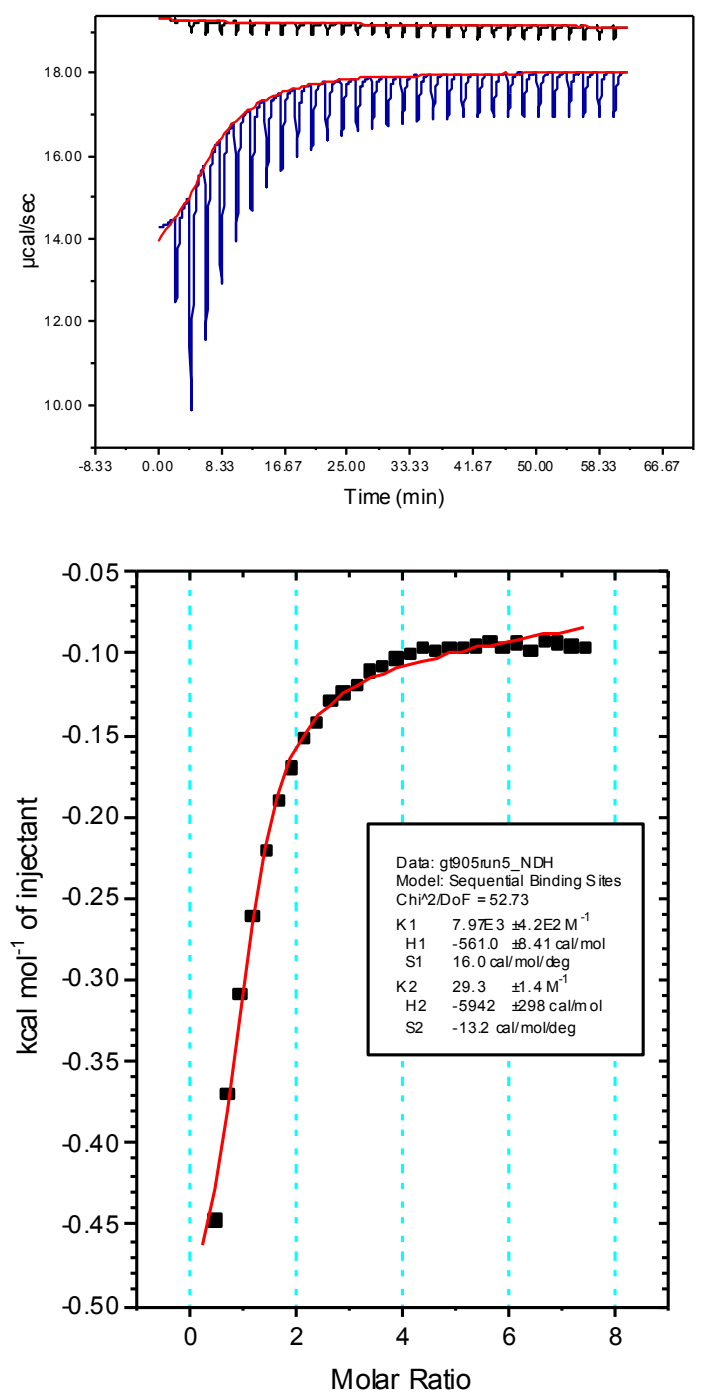

Figure S16: Isothermal titration calorimetry (ITC) curves obtained by titrating $2\left(0.7 \mathrm{mM}, 1 \% \mathrm{DMSO}\right.$ in $\left.\mathrm{H}_{2} \mathrm{O}\right)$ with (2-Hydroxypropyl)- $\beta$-cyclodextrin ( $\mathrm{HP}-\beta-\mathrm{CD})\left(60 \mathrm{mM}\right.$ in $\left.\mathrm{H}_{2} \mathrm{O}\right)$. The top portion of the figure shows the raw data and the bottom curve is the area of the corrected data (dilution heat subtracted). The reference power was set to $20 \mu \mathrm{cal} / \mathrm{sec}$ and the temperature of the cell was maintained at $25^{\circ} \mathrm{C}$.

Note: HP- $\beta-C D$ was preferred over $\beta-C D$ for ITC experiments because of its higher solubility in water. 


\section{ROS production}

The redox cycling ability of the ferrocene moiety in L3, 2@HP- $\beta$-CD and 11@HP- $\beta$-CD was probed using fluorescence spectroscopy. ${ }^{\mathrm{S3}}$ More precisely, in the presence of $\mathrm{H}_{2} \mathrm{O}_{2}(10 \mathrm{mM})$, glutathione $(5 \mathrm{mM})$ and EDTA $(10 \mathrm{mM})$ in PBS, the fluorescence at $531 \mathrm{~nm}$ (excitation at $501 \mathrm{~nm}$ ) resulting from the oxidation of 2 ',7'-dichlorofluorescein dye by reactive oxygen species was monitored overtime (at room temperature). The time is measured from when the drug is added to all the other reagents.
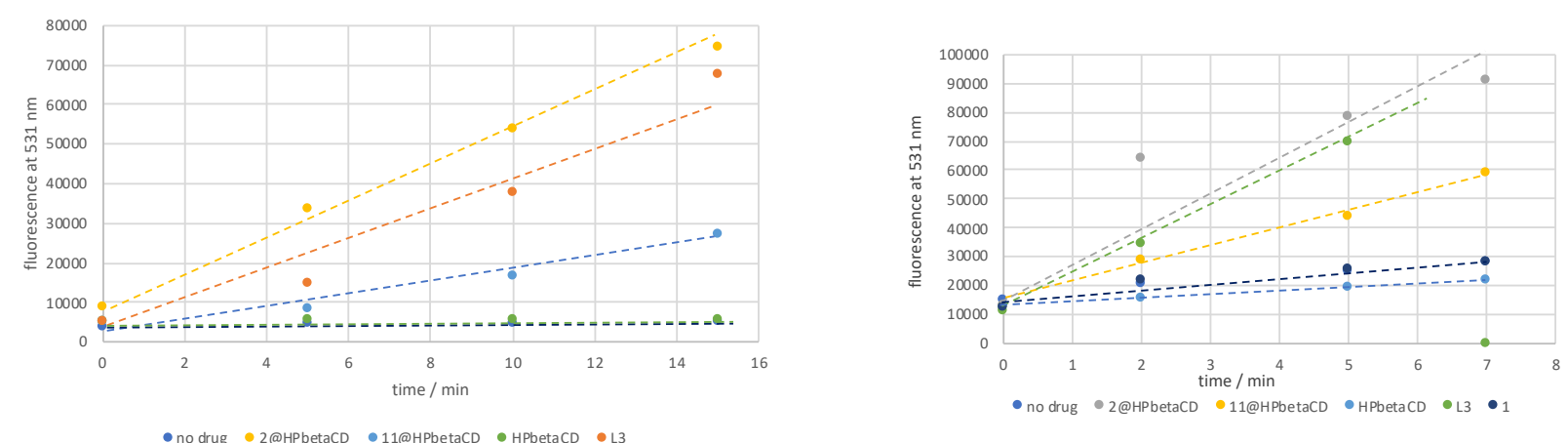

Figure S17: fluorescence emission at $531 \mathrm{~nm}$ monitored overtime upon excitation at $501 \mathrm{~nm}$. The solutions contain glutathione $(5 \mathrm{mM})$, EDTA $(10 \mathrm{mM}), 2^{\prime}, 7^{\prime}$-dichlorofluorescein $(10 \mu \mathrm{M}), \mathrm{H}_{2} \mathrm{O}_{2}(10 \mathrm{mM})$ and the drug of interest ( $[\mathrm{Fc}]=100 \mu \mathrm{M}$ for L3, 2@HP- $\beta-C D$ and 11@HP- $\beta-C D)$ in PBS at room temperature. 


\section{Synthesis of SiR- $\beta-C D$}

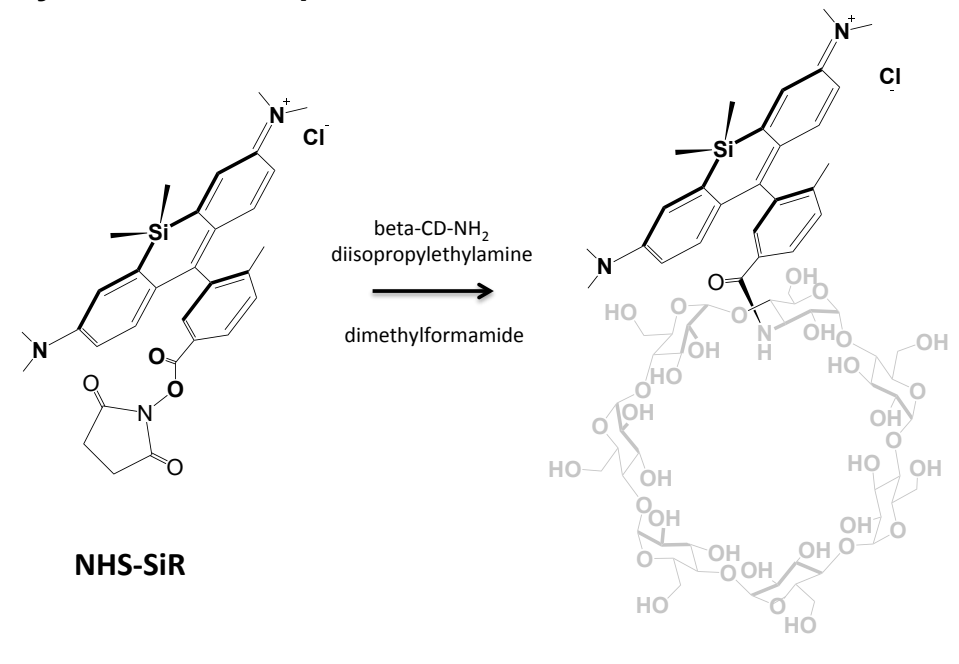

betaCD-SiR

Figure S18: Synthetic scheme showing the route used to prepare SiR- $\boldsymbol{\beta}-\mathbf{C D}$; SiR-NHS was synthesized using the protocol published in ref. [S4].

Figure S18 shows the synthetic scheme used to make SiR- $\beta-C D$ :

SiR was prepared using the protocol established by Johnsson, et al. ${ }^{[\mathrm{S} 4]}$ To a solution of $5 \mathrm{~mL}$ anhydrous methylene chloride under an argon atmosphere charged with SiR $(25 \mathrm{mg}, 0.052 \mathrm{mmol})$ was added Nhydroxysuccinimide (6.6 mg, $0.057 \mathrm{mmol}), \mathrm{N}$-(3-dimethylaminopropyl)-N'-ethylcarbodiimide hydrochloride (10 $\mathrm{mg}, 0.057 \mathrm{mmol})$, and 4-(dimethylamino)pyridine $(0.65 \mathrm{mg}, 0.0052 \mathrm{mmol})$. The mixture was then stirred at room temperature for 4 hours. The crude reaction mixture was washed $3 \times 5 \mathrm{~mL} 0.1 \mathrm{~N} \mathrm{HCl}$ and the resulting aqueous solution was extracted $1 \times 10 \mathrm{~mL}$ DCM. The organic fractions were combined, washed with brine, and dried over $\mathrm{Na}_{2} \mathrm{SO}_{4}$. Following removal of the volatiles on a rotary evaporator, SiR-NHS was isolated as a blue solid; it was used directly in the next step.

SiR-NHS as prepared above was dissolved in dry DMF, 1 equiv of diisopropylethylamine and 3A-amino$3 A$-deoxy-(2AS,3AS)- $\beta$-cyclodextrin were added and the mixture was left stirring in the dark for about $15 \mathrm{~h}$. Volatiles were evaporated off under high vacuum (cold finger). The resulting blue residue was dissolved in $5 \mathrm{ml}$ of water, filtered, and purified using a C18 semi-preparative RP-HPLC system. The fractions containing the pure product SiR- $\boldsymbol{\beta}-\mathbf{C D}$ were combined, concentrated, and finally lyophilized to obtain a cotton-like blue solid. 


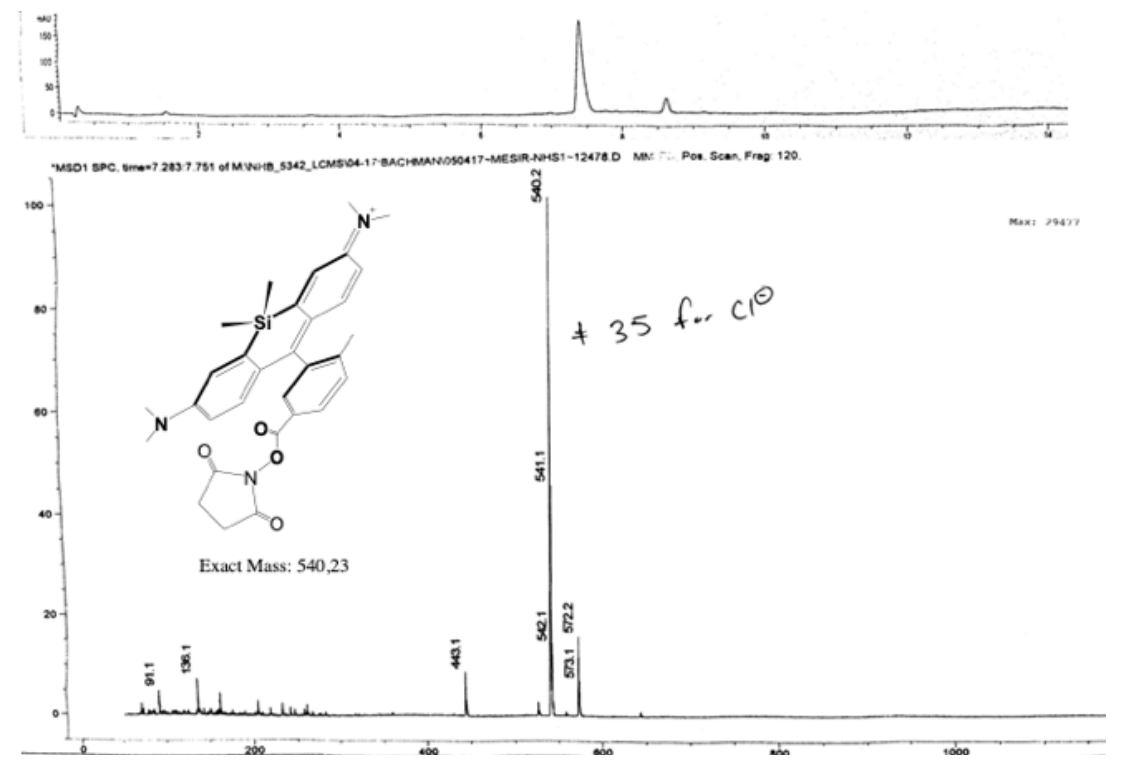

Figure S19: LC (254 nm, top) - MS (ESI, positive mode, low resolution, bottom) profile for SiR-NHS.

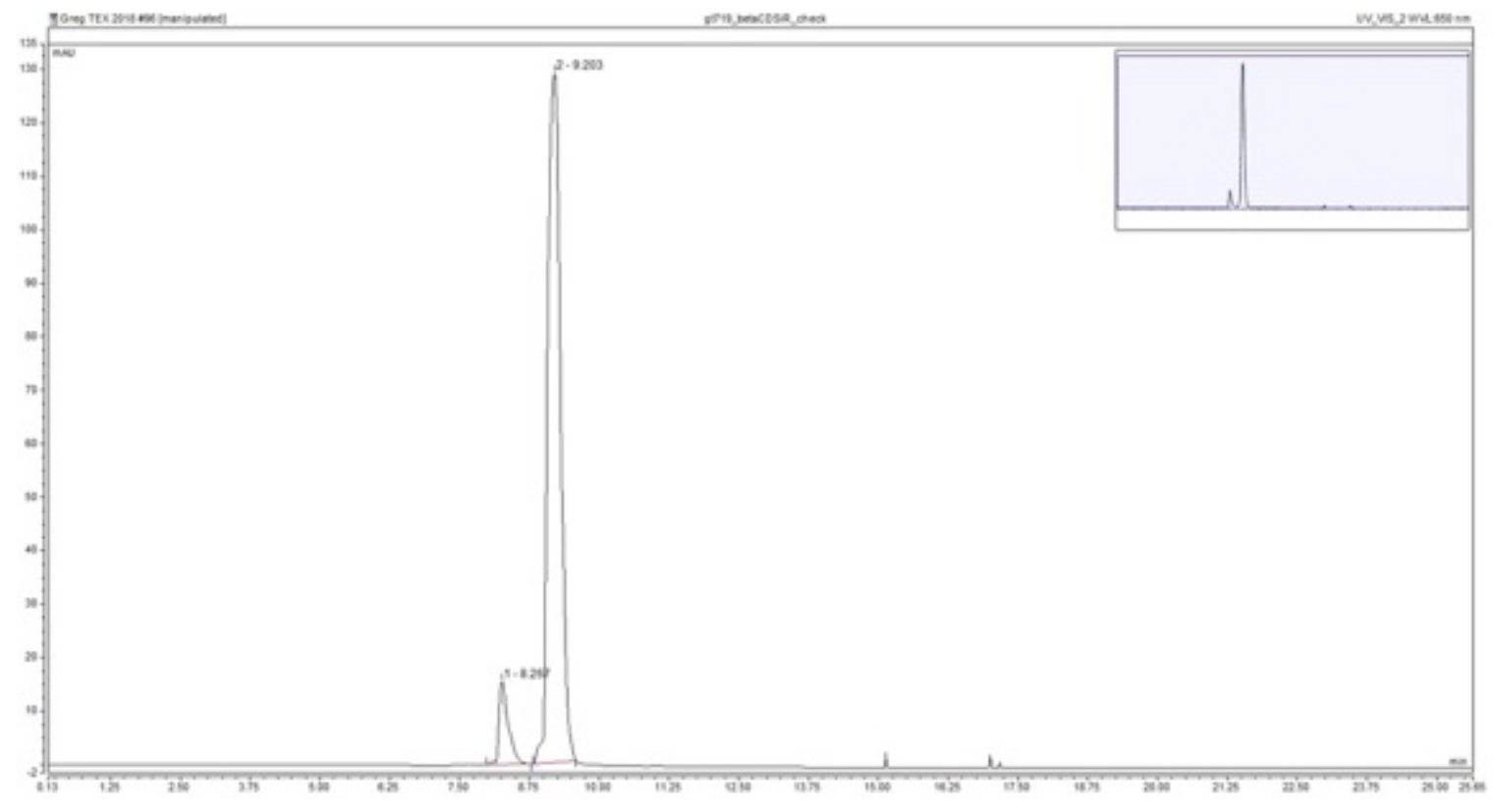

Figure S20: RP-HPLC (detector set at $650 \mathrm{~nm}$ ) chromatogram for SiR- $\beta$-CD after RP-HPLC purification on a semi-preparative C18 column. 


\section{ESI-MS}

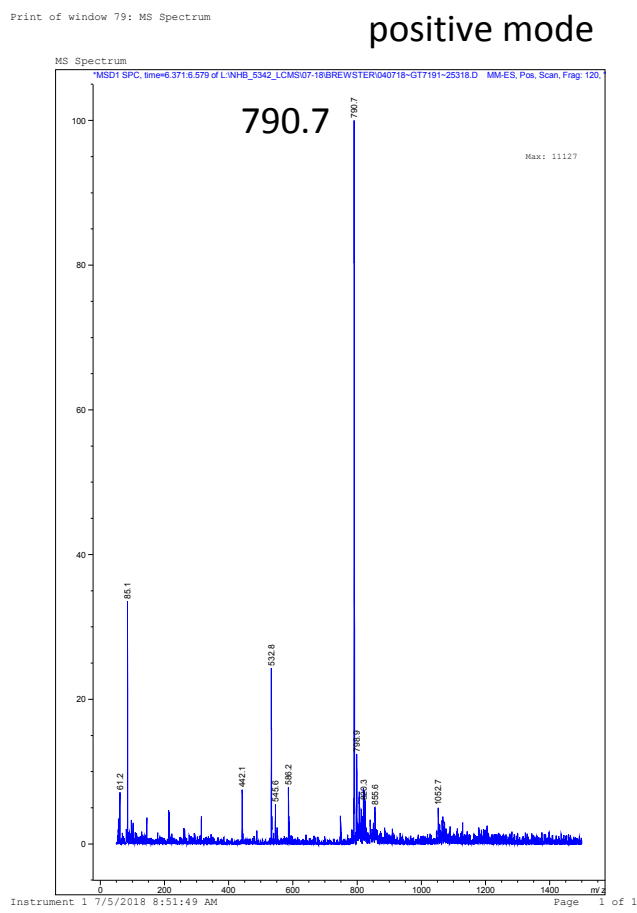

Figure S21: ESI-MS (low resolution) profile of SiR- $\beta$-CD after RP-HPLC purification on a semi-preparative C18 column. The main peak at 790.7 amu is ascribed to the sodium adduct with two positive charges.

Target Compound Screening Report

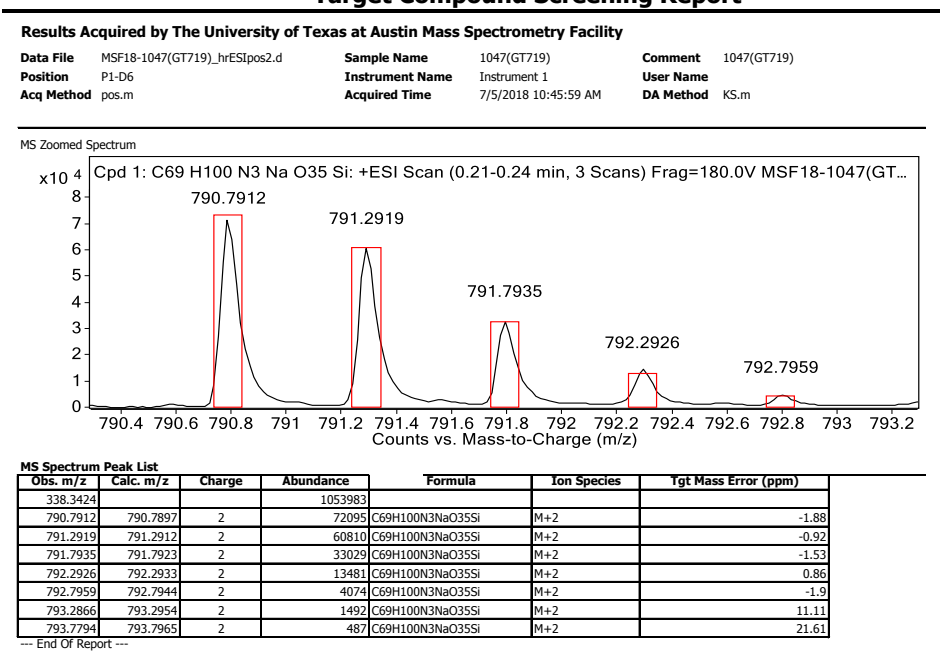

Figure S22: High-resolution mass spectrum showing the isotopic pattern found and calculated for [SiR- $\beta$ $\left.\mathrm{CD}+\mathrm{Na}^{+}\right]^{2+}$. 


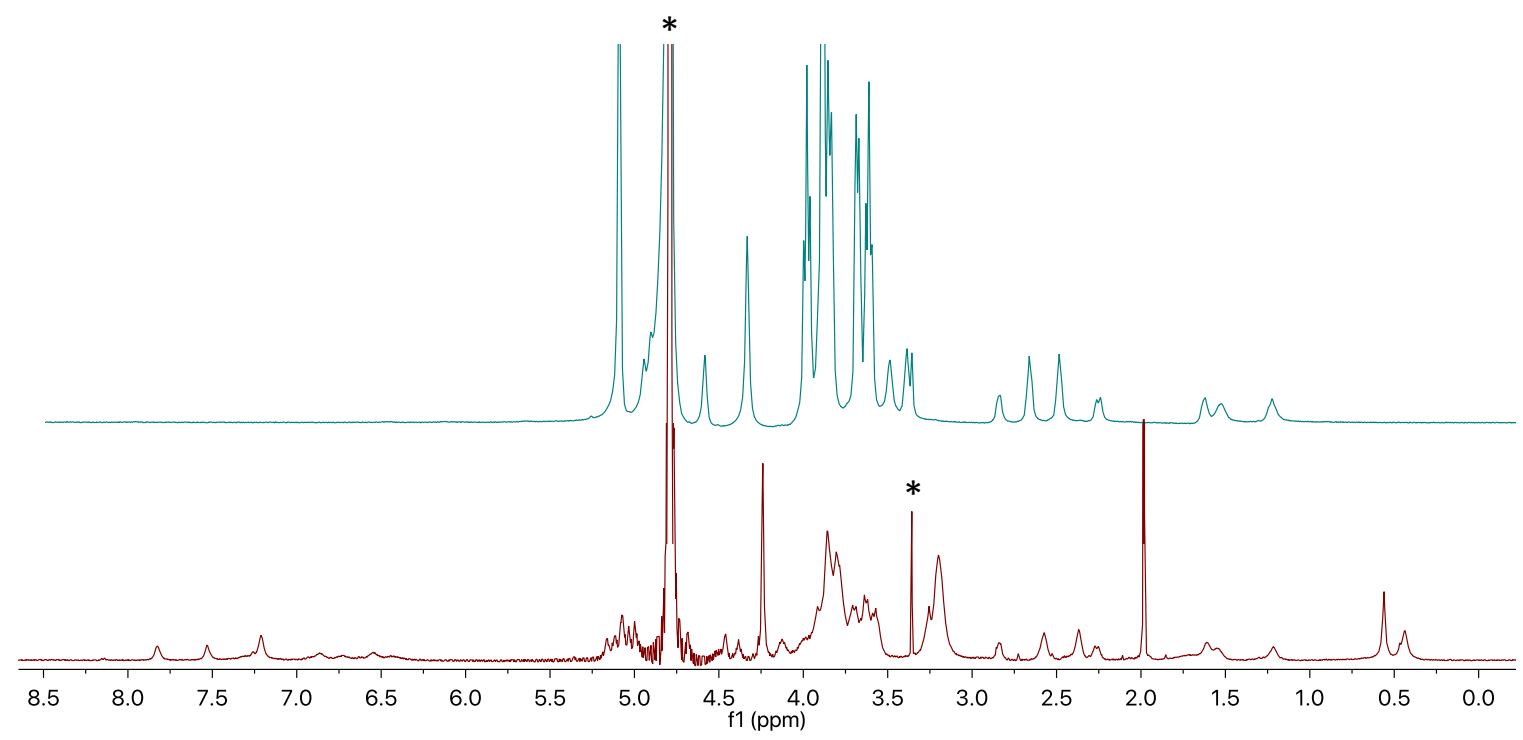

Figure S23: ${ }^{1} \mathrm{H}$ NMR spectra $\left(\mathrm{D}_{2} \mathrm{O}, 300 \mathrm{~K}\right)$ of $\mathbf{2}(3.34 \mathrm{mM})+2.7$ equiv $\boldsymbol{\beta}-\mathrm{CD}$ (top) and $\mathbf{2}(1.7 \mathrm{mM})+2.2$ equiv SiR- $\boldsymbol{\beta}$-CD (bottom). In both cases, 2 dissolved in $100 \%$ water after stirring rapidly for a few minutes. ${ }^{*}$ Residual solvent peaks (methanol and water).

A

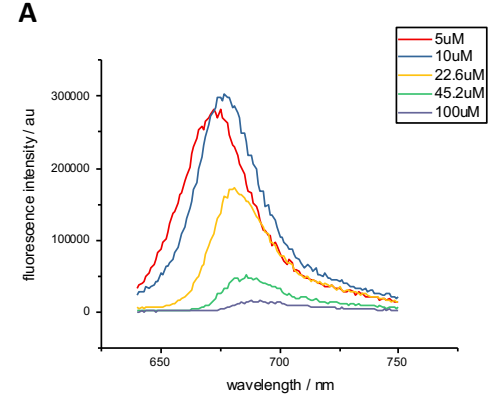

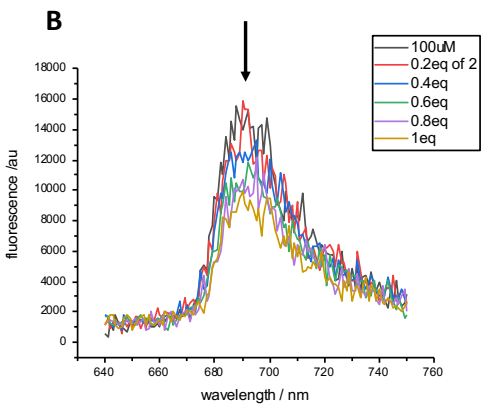

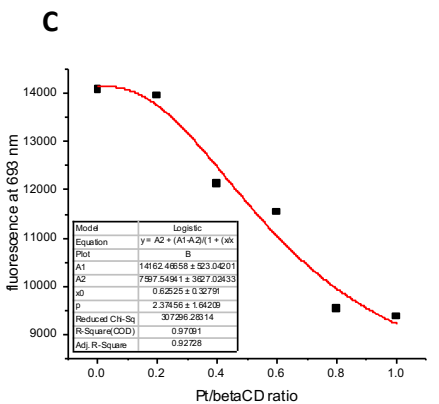

Figure S24: fluorescence spectra (in PBS) of SiR- $\beta-C D$ upon excitation at $645 \mathrm{~nm}$ at different concentrations (A); effect of addition of 2 on the fluorescence emission of SiR- $\boldsymbol{\beta}$-CD (100 $\mu \mathrm{M})(\mathrm{B})$; plot of the fluorescence intensity at $693 \mathrm{~nm}$ against 2 to $\mathrm{SiR}-\boldsymbol{\beta}-\mathrm{CD}$ ratio $(\mathrm{C})$. 


\section{Antiproliferative activity (MTT assays)}
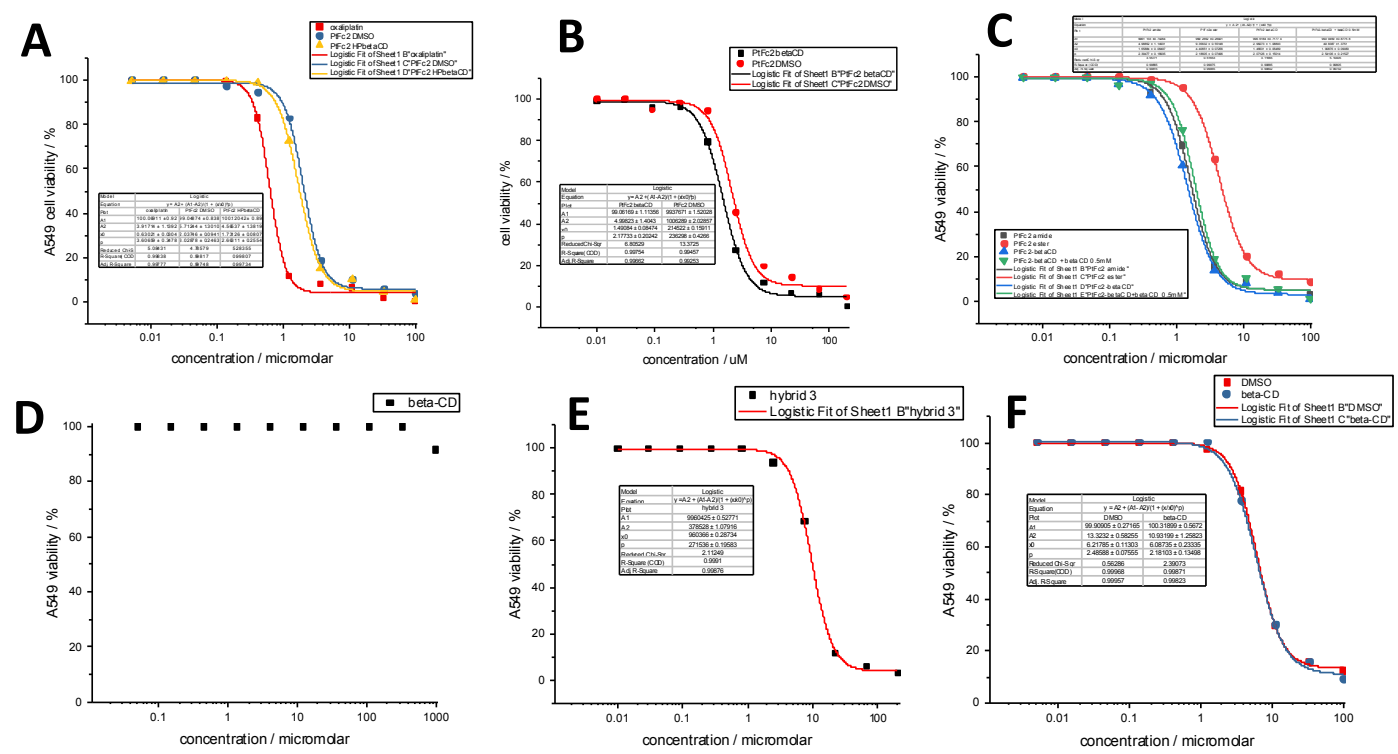

Figure S25: Antiproliferative activities determined by MTT assay in A549 lung cancer cells. Oxaliplatin vs. 2 with DMSO vs. 2 in the presence of HP- $\beta-C D(A), 2$ with DMSO vs. 2 in the presence of $\beta-C D(B), 11$ with DMSO vs. 2 with DMSO vs. 2 in the presence of $\beta-C D$ vs. $2+0.5 \mathrm{mM} \beta-C D(C), \beta-C D(D), 3(E), 11$ with DMSO vs. 11 with $\beta-C D(F)$. Cells were incubated in the presence of the test agent for 5 days.

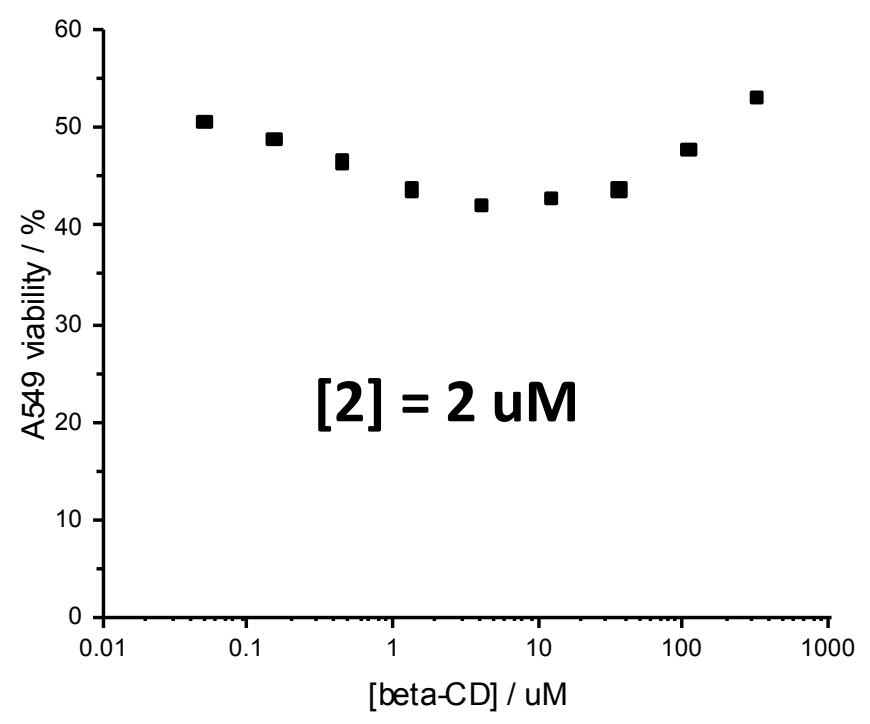

Figure S26: Antiproliferative activity of 2 (fixed concentration $=2 \mu \mathrm{M}$ ) against A549 cancer cells as a function of $[\beta-C D]$. 
Note: both $\beta-C D$ and HP- $\beta-C D$ have been tested against $A 549$ and gave similar results in term of $\mathrm{IC}_{50}$ values.
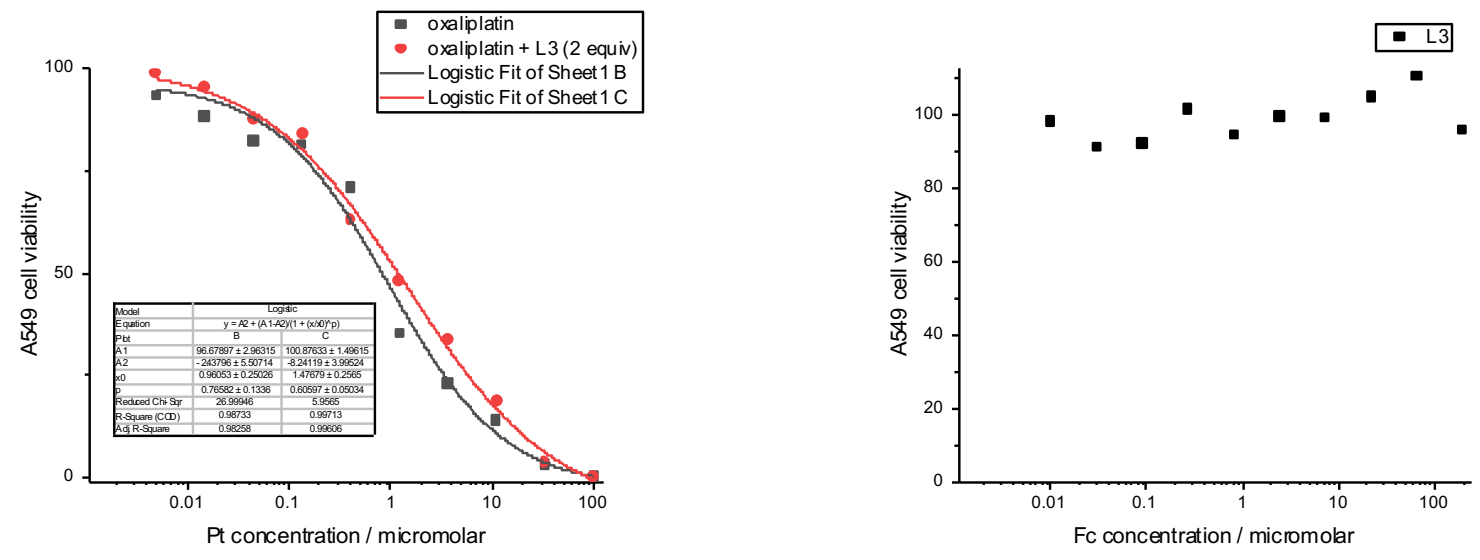

Figure S27: antiproliferative activities of oxaliplatin, oxaliplatin + L3, and L3 against A549 cells evaluated by MTT assay (3-day incubation with the species in question). 


\section{Cellular uptake measurements:}

A549 cells were grown until confluent in T-75 flasks. The agents of interest were added $(100 \mu \mathrm{M})$ and left for varying times. Cells were then washed twice with PBS, submitted to trypsin (1x), counted and pelleted by centrifugation ( $3 \mathrm{~min}$ at $2000 \mathrm{rpm}$ ). After removal of the supernatant, the pellet was suspended/washed one more time with PBS $(5 \mathrm{ml})$ and reformed as a pellet by centrifugation ( $3 \mathrm{~min}$ at $2000 \mathrm{rpm})$. The pellet was then digested in nitric acid $(100 \mu \mathrm{L})$ for $15 \mathrm{~h}$ at $60^{\circ} \mathrm{C}$ and diluted with milliQ water to a final concentration of $2 \% \mathrm{HNO}_{3}$ before being analyzed by ICP-MS.

\section{Fluorescence microscopy:}

A549 cells were incubated at 50000 cells per well per $\mathrm{ml}(\mathrm{V}=1 \mathrm{ml})$ in 24-well plates. Note: A glass cover slip was added to the bottom of each well. Cells were placed on this cover slip. After a defined amount of time, the cells were washed with PBS three times. Paraformaldehyde (4\% in PBS; $500 \mu \mathrm{L})$ was then added into each well and the resulting plate warmed at $37^{\circ} \mathrm{C}$ for $10 \mathrm{~min}$. The cells were washed three times with PBS before adding $500 \mu \mathrm{L}$ PBS to immerse the glass cover slip. The cover slips were then transferred to microscope slides, with cells facing up. A drop of Hoechst dye in solution was then added onto the cover slip before turning it upside down. As the result of this procedure the cells end up in a "sandwich" between the slide and the slip. Some nail polish was applied around the slip to immobilize it. The resulting slides were kept in the dark for 10 min until placed inside the microscope chamber at $37^{\circ} \mathrm{C}$ for observation.

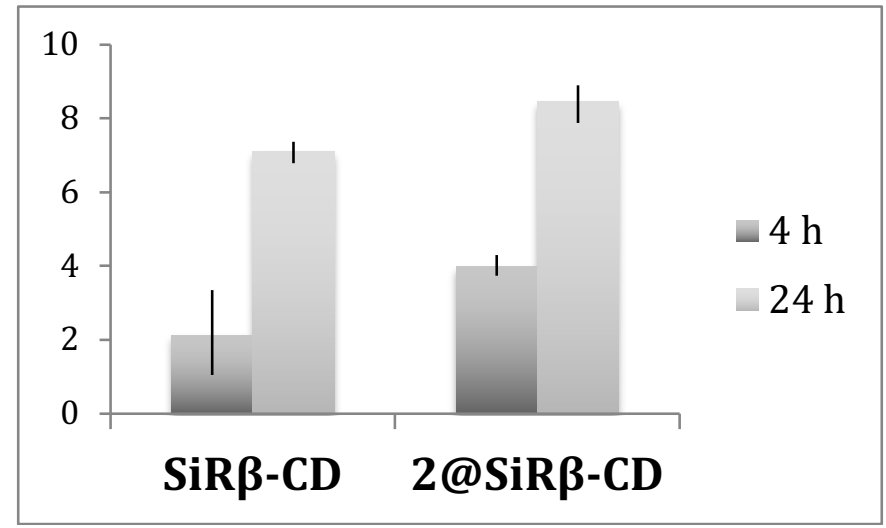

Figure S28: Quantification of the intracellular red fluorescence (using Fiji ImageJ) of A549 cells seen upon incubation with SiR- $\beta-C D$ or $2 @ S i R-\beta-C D$ for 4 or $24 \mathrm{~h}$, respectively. The error bars were calculated by quantifying 3 different images under each set of conditions. 


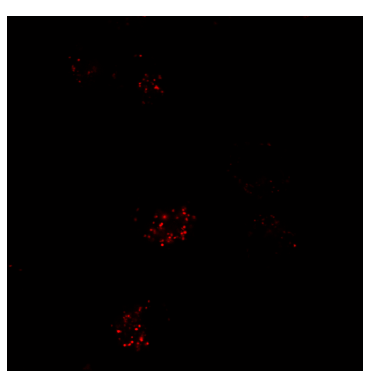

$2 @ S i R-\beta-C D$

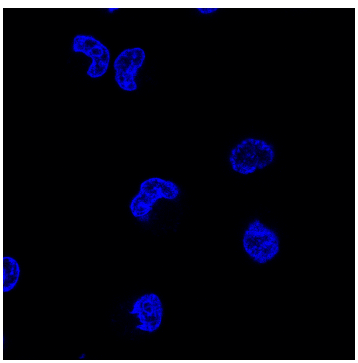

Hoechst 33342

Nucleus dye

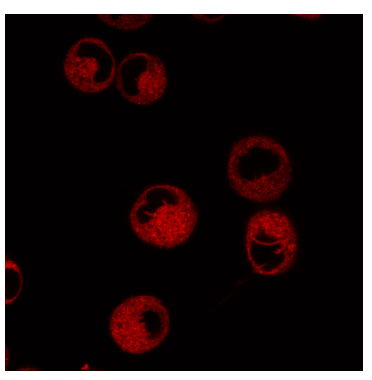

Red ER tracker

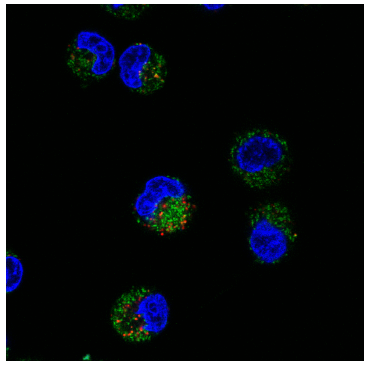

Hoechst 33342 (blue)

MitoTracker (green)

$2 @ S i R-\beta-C D$ (red)

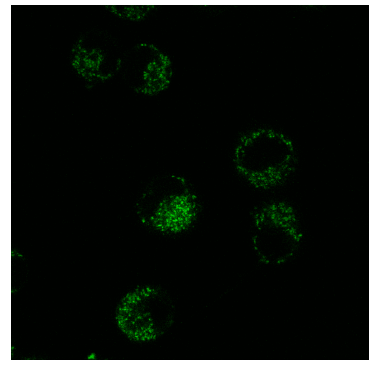

MitoTracker green

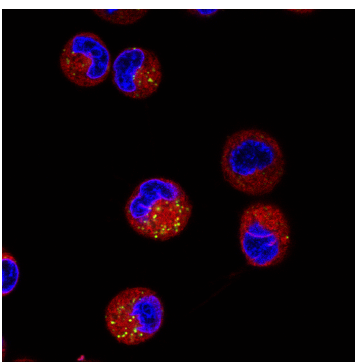

Hoechst 33342 (blue)

ER tracker (red)

2@SiR- $\beta$-CD (green)

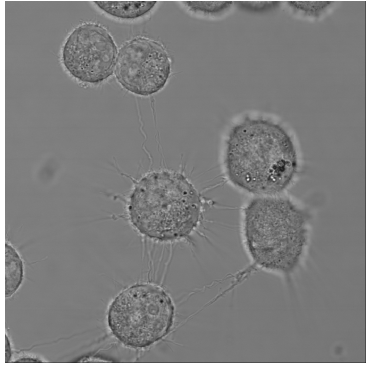

Bright field

Figure S29: fluorescence microscopy of A549 cells incubated for $24 \mathrm{~h}$ in the presence of 2@SiR- $\beta$-CD (50 $\mu \mathrm{M})$, and 20 min prior to analysis with ER tracker, green mitotracker, and nucleus Hoechst 33342 dyes.

\section{X-ray experimental:}

CCDC 1890548 (for 1) and 1890547 (for 2) contain the supplementary crystallographic data for this paper. These data are provided free of charge by the Cambridge Crystallographic Data Centre.

X-ray Experimental for (1) $\left(\mathrm{C}_{4} \mathrm{H}_{5} \mathrm{O}_{2}\right)_{2}^{-}\left(\mathrm{C}_{2} \mathrm{O}_{4}\right)^{2-}\left(\mathrm{C}_{6} \mathrm{H}_{14} \mathrm{~N}_{2}\right) \mathrm{Pt}^{4+}-\mathrm{H}_{2} \mathrm{O}$ : Crystals grew as colorless prisms by slow evaporation from methanol. The data crystal had approximate dimensions; $0.26 \times 0.16 \times 0.14 \mathrm{~mm}$. The data were collected on an Agilent Technologies SuperNova Dual Source diffractometer using a $\mu$-focus Mo $K \alpha$ radiation source $(\lambda=0.71073 \AA$ ) with collimating mirror monochromators. A total of 550 frames of data were collected using $\omega$-scans with a scan range of $1^{\circ}$ and a counting time of 8 seconds per frame. The data were collected at $100 \mathrm{~K}$ using an Oxford 700 Cryostream low temperature device. Details of crystal data, data collection and structure refinement are listed in Table 1. Data collection, unit cell refinement and data reduction were performed using Agilent Technologies CrysAlisPro V 1.171.37.31. ${ }^{\mathrm{S} 5}$ The structure was solved by direct methods using SHELXT's6 and refined by full-matrix least-squares on $\mathrm{F}^{2}$ with anisotropic displacement parameters for the non-H atoms using SHELXL-2016/6. ${ }^{\text {S7 }}$ Structure analysis was aided by use of the programs PLATON98 ${ }^{\mathrm{S8}}$ and WinGX. ${ }^{\mathrm{S9}}$ The hydrogen atoms were calculated in ideal positions with isotropic displacement parameters set to $1.2 \times$ Ueq of the attached atom (1.5xUeq for methyl hydrogen atoms). The absolute configuration was determined using the method of Flack ${ }^{\mathrm{S} 10}$ and confirmed using the Hooft y-parameter method. ${ }^{\mathrm{S} 11}$

Initially, the structure was solved and refined in space group P21/c. There were several hundred low intensity reflections that violated the c centering symmetry operation. In addition, the 1,2-diaminocyclohexane molecule was completely disordered. In space group P21, with two Pt complexes in the asymmetric unit, there is no disorder in these groups. Both 1,2-diamino-cyclohexane molecules have the 
$R, R$ configuration. The absolute structure is well determined. If the crystal was truly centrosymmetric, the diamino ligand would show signs of disorder in both space groups and the absolute structure would be indeterminate with a Flack parameter hovering around 0.5. The hydrogen atoms on the carboxylic acid oxygen atoms and the water oxygen atoms were located in a difference electron density map. These hydrogen atoms were set to riding relative to the attached oxygen atom with displacement parameters set to $1.2 \times$ Ueq or $1.5 \times$ Ueq of the oxygen atoms.

The function, $\Sigma \mathrm{w}\left(\left|\mathrm{F}_{\mathrm{O}}\right|^{2}-\left|\mathrm{F}_{\mathrm{C}}\right|^{2}\right)^{2}$, was minimized, where $\mathrm{w}=1 /\left[\left(\sigma\left(\mathrm{F}_{\mathrm{O}}\right)\right)^{2}+\left(0.0279^{*} \mathrm{P}\right)^{2}+\left(0.1533^{*} \mathrm{P}\right)\right]$ and $P=\left(\left|F_{O}\right|^{2}+2\left|F_{C}\right|^{2}\right) / 3 . R_{W}\left(F^{2}\right)$ refined to 0.0712 , with $R(F)$ equal to 0.0318 and a goodness of fit, $S,=$ 1.11. Definitions used for calculating $R(F), R_{W}\left(F^{2}\right)$ and the goodness of fit, $S$, are given below. ${ }^{S 12}$ The data were corrected for secondary extinction effects. The correction takes the form: $F_{\text {corr }}=\mathrm{kF}_{\mathrm{C}} /\left[1+\left(5.6(9) \times 10^{-}\right.\right.$ $\left.\left.{ }^{7}\right)^{*} F_{c}{ }^{2} \lambda^{3} /(\sin 2 \theta)\right]^{0.25}$ where $k$ is the overall scale factor. Neutral atom scattering factors and values used to calculate the linear absorption coefficient are from the International Tables for X-ray Crystallography (1992). ${ }^{\mathrm{S} 13}$ All figures were generated using SHELXTL/PC. ${ }^{\mathrm{S} 14}$ Tables of positional and thermal parameters, bond lengths and angles, torsion angles and figures may be found in the .cif documents that are archived with the Cambridge Crystallographic Data Centre.

Table S1. Crystal data and structure refinement for 1.

Empirical formula

Formula weight

Temperature

Wavelength

Crystal system

Space group

Unit cell dimensions

Volume

Z

Density (calculated)

Absorption coefficient

$\mathrm{F}(000)$

Crystal size

Theta range for data collection

Index ranges

Reflections collected

Independent reflections

Completeness to theta $=25.242^{\circ}$

Absorption correction
C16 H25 N2 O13 Pt

648.47

100(2) K

$0.71073 \AA$

monoclinic

P 21

$\mathrm{a}=9.1244(3) \AA$

$\alpha=90^{\circ}$.

$\mathrm{b}=11.1445(3) \AA$

$\beta=92.088(3)^{\circ}$.

$\mathrm{c}=20.6956(7) \AA$

$\gamma=90^{\circ}$.

2103.07(11) $\AA^{3}$

4

$2.048 \mathrm{Mg} / \mathrm{m}^{3}$

$6.745 \mathrm{~mm}^{-1}$

1268

$0.260 \times 0.160 \times 0.140 \mathrm{~mm}^{3}$

2.234 to $33.102^{\circ}$.

$-13<=\mathrm{h}<=13,-16<=\mathrm{k}<=17,-30<=\mathrm{k}<=31$

35029

$14087[\mathrm{R}(\mathrm{int})=0.0365]$

$100.0 \%$

Gaussian and multi-scan 
Max. and min. transmission

Refinement method

Data / restraints / parameters

Goodness-of-fit on $\mathrm{F}^{2}$

Final $\mathrm{R}$ indices [I $>2 \operatorname{sigma}(\mathrm{I})]$

$\mathrm{R}$ indices (all data)

Absolute structure parameter

Extinction coefficient

Largest diff. peak and hole
0.799 and 0.389

Full-matrix least-squares on $\mathrm{F}^{2}$

14087 / 385 / 578

1.107

$\mathrm{R} 1=0.0318, \mathrm{wR} 2=0.0689$

$\mathrm{R} 1=0.0350, \mathrm{wR} 2=0.0712$

$0.013(8)$

$0.00056(9)$

1.297 and -1.300 e. $\AA^{-3}$ 
Table S2. Atomic coordinates $\left(x 1^{4}\right)$ and equivalent isotropic displacement parameters $\left(\AA^{2} \times 10^{3}\right)$ for 1 . $U(e q)$ is defined as one third of the trace of the orthogonalized $U^{i j}$ tensor.

\begin{tabular}{|c|c|c|c|c|}
\hline & $\mathrm{x}$ & $\mathrm{y}$ & z & $\mathrm{U}(\mathrm{eq})$ \\
\hline $\mathrm{C} 1$ & $3633(6)$ & $2222(5)$ & $1647(3)$ & $13(1)$ \\
\hline $\mathrm{C} 2$ & $3989(8)$ & $991(7)$ & $1946(4)$ & $16(2)$ \\
\hline $\mathrm{C} 3$ & 2897(8) & $70(6)$ & $1683(4)$ & $24(1)$ \\
\hline $\mathrm{C} 4$ & $2832(8)$ & $33(6)$ & $943(4)$ & $24(2)$ \\
\hline $\mathrm{C} 5$ & $2517(7)$ & 1291(7) & $656(4)$ & $15(2)$ \\
\hline C6 & $3659(6)$ & $2157(5)$ & $918(3)$ & $13(1)$ \\
\hline $\mathrm{C} 7$ & $6203(7)$ & $6614(8)$ & $1573(3)$ & $13(1)$ \\
\hline $\mathrm{C} 8$ & $5610(8)$ & 6799(7) & $873(4)$ & $14(2)$ \\
\hline C9 & $7734(8)$ & $4106(8)$ & 743(4) & $15(2)$ \\
\hline $\mathrm{C} 10$ & $8716(8)$ & $3175(7)$ & $455(4)$ & $17(2)$ \\
\hline C11 & $7861(8)$ & $2089(7)$ & $178(4)$ & $19(2)$ \\
\hline $\mathrm{C} 12$ & $8815(8)$ & 1154(8) & $-116(4)$ & $15(2)$ \\
\hline C13 & $2510(8)$ & $6122(8)$ & $1774(4)$ & $15(2)$ \\
\hline $\mathrm{C} 14$ & $1043(8)$ & $6128(8)$ & 2104(4) & $19(2)$ \\
\hline C15 & $482(7)$ & $7371(8)$ & $2230(4)$ & $16(2)$ \\
\hline $\mathrm{C} 16$ & $1283(8)$ & $8031(8)$ & 2776(4) & $17(2)$ \\
\hline $\mathrm{C} 17$ & $5679(6)$ & $8029(5)$ & $3493(3)$ & $12(1)$ \\
\hline $\mathrm{C} 18$ & $6011(10)$ & $9103(8)$ & $3063(4)$ & $23(2)$ \\
\hline C19 & $6439(8)$ & 10173(6) & $3487(4)$ & $24(1)$ \\
\hline $\mathrm{C} 20$ & $7738(8)$ & $9852(6)$ & $3942(4)$ & $22(2)$ \\
\hline $\mathrm{C} 21$ & $7419(9)$ & 8781(8) & $4362(4)$ & $21(2)$ \\
\hline $\mathrm{C} 22$ & $6972(6)$ & $7705(5)$ & $3934(3)$ & 11(1) \\
\hline $\mathrm{C} 23$ & $3743(7)$ & $3467(7)$ & $3440(3)$ & $12(1)$ \\
\hline $\mathrm{C} 24$ & $4401(8)$ & $3264(7)$ & $4130(3)$ & $12(1)$ \\
\hline $\mathrm{C} 25$ & $2276(8)$ & 5983(7) & $4254(4)$ & 11(1) \\
\hline $\mathrm{C} 26$ & $1319(7)$ & 6934(7) & $4548(3)$ & $12(1)$ \\
\hline $\mathrm{C} 27$ & $2167(8)$ & 7936(8) & 4882(4) & $24(2)$ \\
\hline $\mathrm{C} 28$ & 1252(9) & $8920(9)$ & $5159(4)$ & $19(2)$ \\
\hline $\mathrm{C} 29$ & $7422(9)$ & $3959(7)$ & $3181(4)$ & $14(2)$ \\
\hline $\mathrm{C} 30$ & $8866(8)$ & $3898(7)$ & 2853(4) & $17(2)$ \\
\hline C31 & 9423(8) & 2623(8) & 2756(4) & $21(2)$ \\
\hline
\end{tabular}




\begin{tabular}{|c|c|c|c|c|}
\hline $\mathrm{C} 32$ & $8617(8)$ & $1963(8)$ & $2229(4)$ & $19(2)$ \\
\hline N1 & $4708(6)$ & $3158(6)$ & $1864(3)$ & $13(1)$ \\
\hline $\mathrm{N} 2$ & $3505(6)$ & $3402(6)$ & $656(3)$ & $13(1)$ \\
\hline N3 & $5245(6)$ & $6935(6)$ & $3124(3)$ & $11(1)$ \\
\hline N4 & $6527(6)$ & $6621(6)$ & $4317(3)$ & $9(1)$ \\
\hline O1 & $5916(5)$ & $5579(6)$ & $1834(2)$ & $12(1)$ \\
\hline $\mathrm{O} 2$ & $4862(5)$ & 5923(5) & $608(2)$ & $12(1)$ \\
\hline $\mathrm{O} 3$ & $6861(5)$ & $7403(5)$ & $1859(3)$ & $16(1)$ \\
\hline $\mathrm{O} 4$ & $5804(6)$ & $7762(5)$ & $606(3)$ & $18(1)$ \\
\hline $\mathrm{O} 5$ & $6388(5)$ & $3729(5)$ & $808(3)$ & $13(1)$ \\
\hline O6 & $8177(6)$ & $5098(5)$ & $906(3)$ & $18(1)$ \\
\hline O7 & $8675(7)$ & $76(6)$ & $-9(3)$ & $24(1)$ \\
\hline O8 & 9791(5) & $1579(6)$ & $-510(3)$ & 21(1) \\
\hline O9 & $2843(6)$ & $5005(5)$ & $1613(3)$ & $15(1)$ \\
\hline $\mathrm{O} 10$ & $3241(6)$ & $6985(6)$ & $1689(3)$ & $23(1)$ \\
\hline O11 & $2345(5)$ & $7615(6)$ & $3069(3)$ & $22(1)$ \\
\hline $\mathrm{O} 12$ & $688(6)$ & $9052(6)$ & $2919(3)$ & $26(1)$ \\
\hline $\mathrm{O} 13$ & $2261(7)$ & $10387(6)$ & $3724(3)$ & $39(2)$ \\
\hline O14 & 3994(5) & $4478(6)$ & $3162(2)$ & $11(1)$ \\
\hline O15 & $5154(5)$ & $4168(5)$ & $4370(2)$ & $12(1)$ \\
\hline O16 & $3021(5)$ & $2668(5)$ & $3169(3)$ & $17(1)$ \\
\hline O17 & 4164(6) & $2344(5)$ & $4416(3)$ & $16(1)$ \\
\hline $\mathrm{O} 18$ & $3604(5)$ & $6352(5)$ & $4190(3)$ & $12(1)$ \\
\hline O19 & 1801(6) & $4975(5)$ & 4093(3) & $19(1)$ \\
\hline $\mathrm{O} 20$ & 1381(6) & $9969(5)$ & $5037(3)$ & $18(1)$ \\
\hline $\mathrm{O} 21$ & $273(6)$ & $8503(6)$ & $5563(3)$ & $22(1)$ \\
\hline $\mathrm{O} 22$ & $7110(5)$ & $5060(5)$ & $3353(3)$ & $13(1)$ \\
\hline $\mathrm{O} 23$ & $6637(6)$ & $3079(6)$ & $3263(3)$ & $23(1)$ \\
\hline $\mathrm{O} 24$ & $7662(6)$ & $2369(6)$ & $1885(3)$ & $30(2)$ \\
\hline $\mathrm{O} 25$ & $9057(8)$ & $848(6)$ & $2169(4)$ & $46(2)$ \\
\hline $\mathrm{O} 26$ & 7768(9) & $-419(9)$ & $1277(3)$ & $66(3)$ \\
\hline Pt1 & $4730(1)$ & $4529(1)$ & $1230(1)$ & $9(1)$ \\
\hline $\mathrm{Pt} 2$ & $5244(1)$ & $5539(1)$ & $3748(1)$ & $8(1)$ \\
\hline
\end{tabular}


Table S3. Bond lengths $[\AA \AA]$ and angles $\left[{ }^{\circ}\right]$ for 1 .

\begin{tabular}{|c|c|c|c|}
\hline C1-N1 & $1.490(8)$ & C13-O10 & $1.187(10)$ \\
\hline $\mathrm{C} 1-\mathrm{C} 6$ & $1.511(9)$ & C13-O9 & $1.328(9)$ \\
\hline $\mathrm{C} 1-\mathrm{C} 2$ & $1.535(10)$ & C13-C14 & $1.525(10)$ \\
\hline C1-H01B & 1.00 & C14-C15 & $1.503(11)$ \\
\hline $\mathrm{C} 2-\mathrm{C} 3$ & $1.518(10)$ & $\mathrm{C} 14-\mathrm{H} 14 \mathrm{~A}$ & 0.99 \\
\hline $\mathrm{C} 2-\mathrm{H} 2 \mathrm{~A}$ & 0.99 & C14-H14B & 0.99 \\
\hline $\mathrm{C} 2-\mathrm{H} 2 \mathrm{~B}$ & 0.99 & $\mathrm{C} 15-\mathrm{C} 16$ & $1.514(10)$ \\
\hline $\mathrm{C} 3-\mathrm{C} 4$ & $1.530(10)$ & $\mathrm{C} 15-\mathrm{H} 15 \mathrm{~A}$ & 0.99 \\
\hline $\mathrm{C} 3-\mathrm{H} 3 \mathrm{~A}$ & 0.99 & C15-H15B & 0.99 \\
\hline $\mathrm{C} 3-\mathrm{H} 3 \mathrm{~B}$ & 0.99 & C16-O11 & $1.217(9)$ \\
\hline C4-C5 & $1.546(10)$ & $\mathrm{C} 16-\mathrm{O} 12$ & $1.300(10)$ \\
\hline $\mathrm{C} 4-\mathrm{H} 4 \mathrm{~A}$ & 0.99 & C17-N3 & $1.485(8)$ \\
\hline C4-H4B & 0.99 & $\mathrm{C} 17-\mathrm{C} 22$ & $1.509(8)$ \\
\hline C5-C6 & $1.507(8)$ & $\mathrm{C} 17-\mathrm{C} 18$ & $1.528(10)$ \\
\hline C5-H5A & 0.99 & $\mathrm{C} 17-\mathrm{H} 01 \mathrm{~S}$ & 1.00 \\
\hline C5-H5B & 0.99 & C18-C19 & $1.524(11)$ \\
\hline C6-N2 & $1.494(9)$ & $\mathrm{C} 18-\mathrm{H} 18 \mathrm{~A}$ & 0.99 \\
\hline C6-H01P & 1.00 & C18-H18B & 0.99 \\
\hline $\mathrm{C} 7-\mathrm{O} 3$ & $1.208(9)$ & C19-C20 & $1.529(10)$ \\
\hline C7-O1 & $1.304(10)$ & C19-H19A & 0.99 \\
\hline C7-C8 & $1.542(10)$ & C19-H19B & 0.99 \\
\hline C8-O4 & $1.223(9)$ & $\mathrm{C} 20-\mathrm{C} 21$ & $1.510(11)$ \\
\hline $\mathrm{C} 8-\mathrm{O} 2$ & $1.301(8)$ & $\mathrm{C} 20-\mathrm{H} 20 \mathrm{~A}$ & 0.99 \\
\hline C9-O6 & $1.220(10)$ & $\mathrm{C} 20-\mathrm{H} 20 \mathrm{~B}$ & 0.99 \\
\hline C9-O5 & $1.309(9)$ & $\mathrm{C} 21-\mathrm{C} 22$ & $1.537(10)$ \\
\hline C9-C10 & $1.508(11)$ & $\mathrm{C} 21-\mathrm{H} 21 \mathrm{~A}$ & 0.99 \\
\hline C10-C11 & $1.540(10)$ & $\mathrm{C} 21-\mathrm{H} 21 \mathrm{~B}$ & 0.99 \\
\hline C10-H10A & 0.99 & C22-N4 & $1.509(8)$ \\
\hline C10-H10B & 0.99 & $\mathrm{C} 22-\mathrm{H} 01 \mathrm{~F}$ & 1.00 \\
\hline $\mathrm{C} 11-\mathrm{C} 12$ & $1.501(11)$ & $\mathrm{C} 23-\mathrm{O} 16$ & $1.230(8)$ \\
\hline C11-H11A & 0.99 & $\mathrm{C} 23-\mathrm{O} 14$ & $1.289(9)$ \\
\hline C11-H11B & 0.99 & $\mathrm{C} 23-\mathrm{C} 24$ & $1.546(9)$ \\
\hline $\mathrm{C} 12-\mathrm{O} 7$ & $1.229(10)$ & C24-O17 & $1.208(9)$ \\
\hline $\mathrm{C} 12-\mathrm{O} 8$ & $1.317(10)$ & $\mathrm{C} 24-\mathrm{O} 15$ & $1.307(9)$ \\
\hline
\end{tabular}




\begin{tabular}{|c|c|c|c|}
\hline $\mathrm{C} 25-\mathrm{O} 19$ & $1.244(9)$ & N1-H1NB & 0.91 \\
\hline $\mathrm{C} 25-\mathrm{O} 18$ & $1.291(8)$ & N2-Pt1 & $2.035(6)$ \\
\hline $\mathrm{C} 25-\mathrm{C} 26$ & $1.515(10)$ & N2-H2NA & 0.91 \\
\hline $\mathrm{C} 26-\mathrm{C} 27$ & $1.511(11)$ & $\mathrm{N} 2-\mathrm{H} 2 \mathrm{NB}$ & 0.91 \\
\hline C26-H26A & 0.99 & N3-Pt2 & $2.022(6)$ \\
\hline $\mathrm{C} 26-\mathrm{H} 26 \mathrm{~B}$ & 0.99 & N3-H3NA & 0.91 \\
\hline $\mathrm{C} 27-\mathrm{C} 28$ & $1.505(12)$ & N3-H3NB & 0.91 \\
\hline C27-H27A & 0.99 & N4-Pt2 & $2.029(5)$ \\
\hline $\mathrm{C} 27-\mathrm{H} 27 \mathrm{~B}$ & 0.99 & N4-H4NA & 0.91 \\
\hline $\mathrm{C} 28-\mathrm{O} 20$ & $1.202(10)$ & N4-H4NB & 0.91 \\
\hline $\mathrm{C} 28-\mathrm{O} 21$ & $1.330(10)$ & O1-Pt1 & $2.001(5)$ \\
\hline C29-O23 & $1.230(10)$ & O2-Pt1 & $2.025(5)$ \\
\hline $\mathrm{C} 29-\mathrm{O} 22$ & $1.312(9)$ & O5-Pt1 & $1.986(5)$ \\
\hline C29-C30 & $1.505(10)$ & O8-H8O & 0.88 \\
\hline C30-C31 & $1.525(12)$ & O9-Pt1 & $1.992(5)$ \\
\hline C30-H30A & 0.99 & O13-H13A & 0.90 \\
\hline C30-H30B & 0.99 & O13-H13B & 0.84 \\
\hline C31-C32 & $1.487(10)$ & O14-Pt2 & $2.016(5)$ \\
\hline C31-H31A & 0.99 & $\mathrm{O} 15-\mathrm{Pt} 2$ & $2.002(5)$ \\
\hline C31-H31B & 0.99 & O18-Pt2 & $1.999(5)$ \\
\hline $\mathrm{C} 32-\mathrm{O} 24$ & $1.194(9)$ & $\mathrm{O} 21-\mathrm{H} 21 \mathrm{O}$ & 0.85 \\
\hline $\mathrm{C} 32-\mathrm{O} 25$ & $1.313(10)$ & $\mathrm{O} 22-\mathrm{Pt} 2$ & $1.988(5)$ \\
\hline N1-Pt1 & $2.014(6)$ & $\mathrm{O} 26-\mathrm{H} 26 \mathrm{C}$ & 0.90 \\
\hline N1-H1NA & 0.91 & $\mathrm{O} 26-\mathrm{H} 26 \mathrm{D}$ & 0.93 \\
\hline N1-C1-C6 & $107.5(5)$ & $\mathrm{H} 2 \mathrm{~A}-\mathrm{C} 2-\mathrm{H} 2 \mathrm{~B}$ & 108.2 \\
\hline $\mathrm{N} 1-\mathrm{C} 1-\mathrm{C} 2$ & $112.2(5)$ & $\mathrm{C} 2-\mathrm{C} 3-\mathrm{C} 4$ & $112.3(6)$ \\
\hline $\mathrm{C} 6-\mathrm{C} 1-\mathrm{C} 2$ & $110.5(5)$ & $\mathrm{C} 2-\mathrm{C} 3-\mathrm{H} 3 \mathrm{~A}$ & 109.1 \\
\hline N1-C1-H01B & 108.9 & C4-C3-H3A & 109.1 \\
\hline C6-C1-H01B & 108.9 & C2-C3-H3B & 109.1 \\
\hline C2-C1-H01B & 108.9 & C4-C3-H3B & 109.1 \\
\hline $\mathrm{C} 3-\mathrm{C} 2-\mathrm{C} 1$ & $109.4(6)$ & $\mathrm{H} 3 \mathrm{~A}-\mathrm{C} 3-\mathrm{H} 3 \mathrm{~B}$ & 107.9 \\
\hline $\mathrm{C} 3-\mathrm{C} 2-\mathrm{H} 2 \mathrm{~A}$ & 109.8 & $\mathrm{C} 3-\mathrm{C} 4-\mathrm{C} 5$ & 111.1(6) \\
\hline $\mathrm{C} 1-\mathrm{C} 2-\mathrm{H} 2 \mathrm{~A}$ & 109.8 & $\mathrm{C} 3-\mathrm{C} 4-\mathrm{H} 4 \mathrm{~A}$ & 109.4 \\
\hline $\mathrm{C} 3-\mathrm{C} 2-\mathrm{H} 2 \mathrm{~B}$ & 109.8 & C5-C4-H4A & 109.4 \\
\hline $\mathrm{C} 1-\mathrm{C} 2-\mathrm{H} 2 \mathrm{~B}$ & 109.8 & C3-C4-H4B & 109.4 \\
\hline
\end{tabular}




\begin{tabular}{|c|c|c|c|}
\hline C5-C4-H4B & 109.4 & $\mathrm{O} 7-\mathrm{C} 12-\mathrm{C} 11$ & $122.7(8)$ \\
\hline $\mathrm{H} 4 \mathrm{~A}-\mathrm{C} 4-\mathrm{H} 4 \mathrm{~B}$ & 108.0 & $\mathrm{O} 8-\mathrm{C} 12-\mathrm{C} 11$ & $114.7(7)$ \\
\hline C6-C5-C4 & $109.1(5)$ & O10-C13-O9 & $126.0(7)$ \\
\hline C6-C5-H5A & 109.9 & $\mathrm{O} 10-\mathrm{C} 13-\mathrm{C} 14$ & $124.8(8)$ \\
\hline C4-C5-H5A & 109.9 & O9-C13-C14 & $109.2(7)$ \\
\hline C6-C5-H5B & 109.9 & C15-C14-C13 & $113.0(7)$ \\
\hline C4-C5-H5B & 109.9 & C15-C14-H14A & 109.0 \\
\hline H5A-C5-H5B & 108.3 & C13-C14-H14A & 109.0 \\
\hline N2-C6-C5 & $114.2(5)$ & C15-C14-H14B & 109.0 \\
\hline N2-C6-C1 & $108.2(5)$ & C13-C14-H14B & 109.0 \\
\hline C5-C6-C1 & $110.7(5)$ & H14A-C14-H14B & 107.8 \\
\hline N2-C6-H01P & 107.8 & C14-C15-C16 & $114.8(6)$ \\
\hline C5-C6-H01P & 107.8 & C14-C15-H15A & 108.6 \\
\hline C1-C6-H01P & 107.8 & C16-C15-H15A & 108.6 \\
\hline $\mathrm{O} 3-\mathrm{C} 7-\mathrm{O} 1$ & $123.0(6)$ & C14-C15-H15B & 108.6 \\
\hline $\mathrm{O} 3-\mathrm{C} 7-\mathrm{C} 8$ & $121.0(7)$ & C16-C15-H15B & 108.6 \\
\hline $\mathrm{O} 1-\mathrm{C} 7-\mathrm{C} 8$ & $116.0(6)$ & H15A-C15-H15B & 107.5 \\
\hline $\mathrm{O} 4-\mathrm{C} 8-\mathrm{O} 2$ & $123.4(7)$ & $\mathrm{O} 11-\mathrm{C} 16-\mathrm{O} 12$ & $123.5(8)$ \\
\hline $\mathrm{O} 4-\mathrm{C} 8-\mathrm{C} 7$ & $119.3(6)$ & O11-C16-C15 & $122.8(8)$ \\
\hline $\mathrm{O} 2-\mathrm{C} 8-\mathrm{C} 7$ & $117.2(7)$ & O12-C16-C15 & $113.7(7)$ \\
\hline O6-C9-O5 & $124.4(7)$ & N3-C17-C22 & $107.6(5)$ \\
\hline O6-C9-C10 & $122.4(7)$ & N3-C17-C18 & $113.5(5)$ \\
\hline $\mathrm{O} 5-\mathrm{C} 9-\mathrm{C} 10$ & $113.2(7)$ & C22-C17-C18 & $111.8(5)$ \\
\hline C9-C10-C11 & $112.8(6)$ & N3-C17-H01S & 107.9 \\
\hline C9-C10-H10A & 109.0 & C22-C17-H01S & 107.9 \\
\hline C11-C10-H10A & 109.0 & C18-C17-H01S & 107.9 \\
\hline C9-C10-H10B & 109.0 & C19-C18-C17 & $109.2(6)$ \\
\hline C11-C10-H10B & 109.0 & C19-C18-H18A & 109.8 \\
\hline H10A-C10-H10B & 107.8 & C17-C18-H18A & 109.8 \\
\hline C12-C11-C10 & $113.8(6)$ & C19-C18-H18B & 109.8 \\
\hline C12-C11-H11A & 108.8 & C17-C18-H18B & 109.8 \\
\hline C10-C11-H11A & 108.8 & H18A-C18-H18B & 108.3 \\
\hline C12-C11-H11B & 108.8 & C18-C19-C20 & $110.4(6)$ \\
\hline C10-C11-H11B & 108.8 & C18-C19-H19A & 109.6 \\
\hline H11A-C11-H11B & 107.7 & C20-C19-H19A & 109.6 \\
\hline O7-C12-O8 & $122.7(8)$ & C18-C19-H19B & 109.6 \\
\hline
\end{tabular}




\begin{tabular}{|c|c|c|c|}
\hline C20-C19-H19B & 109.6 & $\mathrm{C} 28-\mathrm{C} 27-\mathrm{H} 27 \mathrm{~A}$ & 108.4 \\
\hline H19A-C19-H19B & 108.1 & C26-C27-H27A & 108.4 \\
\hline C21-C20-C19 & $112.2(6)$ & C28-C27-H27B & 108.4 \\
\hline $\mathrm{C} 21-\mathrm{C} 20-\mathrm{H} 20 \mathrm{~A}$ & 109.2 & C26-C27-H27B & 108.4 \\
\hline C19-C20-H20A & 109.2 & $\mathrm{H} 27 \mathrm{~A}-\mathrm{C} 27-\mathrm{H} 27 \mathrm{~B}$ & 107.5 \\
\hline C21-C20-H20B & 109.2 & $\mathrm{O} 20-\mathrm{C} 28-\mathrm{O} 21$ & $123.0(9)$ \\
\hline C19-C20-H20B & 109.2 & $\mathrm{O} 20-\mathrm{C} 28-\mathrm{C} 27$ & 124.7(9) \\
\hline $\mathrm{H} 20 \mathrm{~A}-\mathrm{C} 20-\mathrm{H} 20 \mathrm{~B}$ & 107.9 & $\mathrm{O} 21-\mathrm{C} 28-\mathrm{C} 27$ & $112.3(8)$ \\
\hline $\mathrm{C} 20-\mathrm{C} 21-\mathrm{C} 22$ & $109.7(6)$ & $\mathrm{O} 23-\mathrm{C} 29-\mathrm{O} 22$ & $125.1(7)$ \\
\hline $\mathrm{C} 20-\mathrm{C} 21-\mathrm{H} 21 \mathrm{~A}$ & 109.7 & $\mathrm{O} 23-\mathrm{C} 29-\mathrm{C} 30$ & $123.4(7)$ \\
\hline $\mathrm{C} 22-\mathrm{C} 21-\mathrm{H} 21 \mathrm{~A}$ & 109.7 & $\mathrm{O} 22-\mathrm{C} 29-\mathrm{C} 30$ & $111.5(7)$ \\
\hline C20-C21-H21B & 109.7 & C29-C30-C31 & $113.7(7)$ \\
\hline $\mathrm{C} 22-\mathrm{C} 21-\mathrm{H} 21 \mathrm{~B}$ & 109.7 & C29-C30-H30A & 108.8 \\
\hline $\mathrm{H} 21 \mathrm{~A}-\mathrm{C} 21-\mathrm{H} 21 \mathrm{~B}$ & 108.2 & C31-C30-H30A & 108.8 \\
\hline N4-C22-C17 & $106.9(5)$ & C29-C30-H30B & 108.8 \\
\hline N4-C22-C21 & $113.1(5)$ & C31-C30-H30B & 108.8 \\
\hline C17-C22-C21 & $110.3(5)$ & H30A-C30-H30B & 107.7 \\
\hline N4-C22-H01F & 108.8 & C32-C31-C30 & $113.5(6)$ \\
\hline C17-C22-H01F & 108.8 & C32-C31-H31A & 108.9 \\
\hline $\mathrm{C} 21-\mathrm{C} 22-\mathrm{H} 01 \mathrm{~F}$ & 108.8 & C30-C31-H31A & 108.9 \\
\hline O16-C23-O14 & $122.0(6)$ & C32-C31-H31B & 108.9 \\
\hline $\mathrm{O} 16-\mathrm{C} 23-\mathrm{C} 24$ & $119.9(7)$ & C30-C31-H31B & 108.9 \\
\hline $\mathrm{O} 14-\mathrm{C} 23-\mathrm{C} 24$ & $118.0(6)$ & H31A-C31-H31B & 107.7 \\
\hline O17-C24-O15 & $124.6(6)$ & $\mathrm{O} 24-\mathrm{C} 32-\mathrm{O} 25$ & $121.4(8)$ \\
\hline O17-C24-C23 & $120.5(7)$ & $\mathrm{O} 24-\mathrm{C} 32-\mathrm{C} 31$ & $125.5(8)$ \\
\hline $\mathrm{O} 15-\mathrm{C} 24-\mathrm{C} 23$ & $114.8(6)$ & $\mathrm{O} 25-\mathrm{C} 32-\mathrm{C} 31$ & 113.1(7) \\
\hline O19-C25-O18 & $125.4(7)$ & C1-N1-Pt1 & $110.9(4)$ \\
\hline $\mathrm{O} 19-\mathrm{C} 25-\mathrm{C} 26$ & $122.6(7)$ & C1-N1-H1NA & 109.5 \\
\hline $\mathrm{O} 18-\mathrm{C} 25-\mathrm{C} 26$ & $112.0(6)$ & Pt1-N1-H1NA & 109.5 \\
\hline C27-C26-C25 & $114.1(5)$ & C1-N1-H1NB & 109.5 \\
\hline $\mathrm{C} 27-\mathrm{C} 26-\mathrm{H} 26 \mathrm{~A}$ & 108.7 & Pt1-N1-H1NB & 109.5 \\
\hline $\mathrm{C} 25-\mathrm{C} 26-\mathrm{H} 26 \mathrm{~A}$ & 108.7 & H1NA-N1-H1NB & 108.0 \\
\hline C27-C26-H26B & 108.7 & C6-N2-Pt1 & $108.6(4)$ \\
\hline C25-C26-H26B & 108.7 & C6-N2-H2NA & 110.0 \\
\hline H26A-C26-H26B & 107.6 & Pt1-N2-H2NA & 110.0 \\
\hline $\mathrm{C} 28-\mathrm{C} 27-\mathrm{C} 26$ & $115.6(6)$ & C6-N2-H2NB & 110.0 \\
\hline
\end{tabular}




\begin{tabular}{|c|c|c|c|}
\hline Pt1-N2-H2NB & 110.0 & O9-Pt1-O1 & $93.0(2)$ \\
\hline H2NA-N2-H2NB & 108.4 & O5-Pt1-N1 & $88.4(2)$ \\
\hline C17-N3-Pt2 & $108.1(4)$ & O9-Pt1-N1 & $85.1(2)$ \\
\hline C17-N3-H3NA & 110.1 & O1-Pt1-N1 & $93.2(2)$ \\
\hline Pt2-N3-H3NA & 110.1 & O5-Pt1-O2 & $90.0(2)$ \\
\hline C17-N3-H3NB & 110.1 & O9-Pt1-O2 & $96.9(2)$ \\
\hline Pt2-N3-H3NB & 110.1 & O1-Pt1-O2 & $84.5(2)$ \\
\hline H3NA-N3-H3NB & 108.4 & N1-Pt1-O2 & $177.0(2)$ \\
\hline C22-N4-Pt2 & $109.3(4)$ & O5-Pt1-N2 & $82.9(2)$ \\
\hline C22-N4-H4NA & 109.8 & O9-Pt1-N2 & $86.1(2)$ \\
\hline Pt2-N4-H4NA & 109.8 & O1-Pt1-N2 & $177.0(2)$ \\
\hline C22-N4-H4NB & 109.8 & N1-Pt1-N2 & $83.9(2)$ \\
\hline Pt2-N4-H4NB & 109.8 & O2-Pt1-N2 & $98.4(2)$ \\
\hline H4NA-N4-H4NB & 108.3 & O22-Pt2-O18 & $167.5(2)$ \\
\hline C7-O1-Pt1 & $111.8(4)$ & $\mathrm{O} 22-\mathrm{Pt} 2-\mathrm{O} 15$ & $96.6(2)$ \\
\hline C8-O2-Pt1 & $110.5(4)$ & O18-Pt2-O15 & $90.1(2)$ \\
\hline C9-O5-Pt1 & $129.3(5)$ & O22-Pt2-O14 & $94.0(2)$ \\
\hline C12-O8-H8O & 108.4 & O18-Pt2-O14 & $97.2(2)$ \\
\hline C13-O9-Pt1 & $123.9(5)$ & O15-Pt2-O14 & $84.5(2)$ \\
\hline H13A-O13-H13B & 113.0 & O22-Pt2-N3 & $85.6(2)$ \\
\hline C23-O14-Pt2 & $110.5(4)$ & O18-Pt2-N3 & $87.8(2)$ \\
\hline C24-O15-Pt2 & $112.1(4)$ & O15-Pt2-N3 & $177.6(2)$ \\
\hline C25-O18-Pt2 & $128.5(5)$ & O14-Pt2-N3 & $94.7(2)$ \\
\hline $\mathrm{C} 28-\mathrm{O} 21-\mathrm{H} 21 \mathrm{O}$ & 106.7 & O22-Pt2-N4 & $85.0(2)$ \\
\hline C29-O22-Pt2 & $124.1(5)$ & O18-Pt2-N4 & $83.7(2)$ \\
\hline $\mathrm{H} 26 \mathrm{C}-\mathrm{O} 26-\mathrm{H} 26 \mathrm{D}$ & 106.4 & O15-Pt2-N4 & $96.7(2)$ \\
\hline O5-Pt1-O9 & $167.8(2)$ & O14-Pt2-N4 & $178.6(2)$ \\
\hline O5-Pt1-O1 & $97.7(2)$ & N3-Pt2-N4 & $84.2(2)$ \\
\hline
\end{tabular}


Table S4. Anisotropic displacement parameters $\left(\AA^{2} \times 10^{3}\right)$ for 1 . The anisotropic displacement factor exponent takes the form: $-2 \pi^{2}\left[h^{2} a^{* 2} U^{11}+\ldots+2 h k a^{*} b^{*} U^{12}\right]$

\begin{tabular}{|c|c|c|c|c|c|c|}
\hline & $\mathrm{U}^{11}$ & $\mathrm{U}^{22}$ & $\mathrm{U}^{33}$ & $U^{23}$ & $\mathrm{U}^{13}$ & $\mathrm{U}^{12}$ \\
\hline $\mathrm{C} 1$ & $7(2)$ & $15(3)$ & $17(3)$ & $0(2)$ & $1(2)$ & $0(2)$ \\
\hline $\mathrm{C} 2$ & 21(3) & 11(3) & $16(3)$ & $5(3)$ & $4(3)$ & $2(3)$ \\
\hline $\mathrm{C} 3$ & $29(3)$ & $17(3)$ & $25(4)$ & $4(3)$ & $4(3)$ & $-4(3)$ \\
\hline $\mathrm{C} 4$ & $29(4)$ & $15(3)$ & $29(4)$ & $0(3)$ & $-2(3)$ & $-7(3)$ \\
\hline C5 & $16(3)$ & $9(3)$ & $20(3)$ & $-5(3)$ & $2(3)$ & $-2(2)$ \\
\hline C6 & $14(3)$ & $13(3)$ & 11(3) & $-1(2)$ & $-1(2)$ & $2(2)$ \\
\hline $\mathrm{C} 7$ & $13(3)$ & $20(4)$ & $7(3)$ & $3(3)$ & $1(2)$ & $5(3)$ \\
\hline $\mathrm{C} 8$ & $11(3)$ & $9(3)$ & 21(4) & $-5(3)$ & $1(3)$ & $-3(2)$ \\
\hline C9 & $12(3)$ & $23(4)$ & $10(3)$ & $-1(3)$ & $0(3)$ & $7(3)$ \\
\hline $\mathrm{C} 10$ & $10(3)$ & $17(4)$ & 24(4) & $-3(3)$ & $0(3)$ & $-1(3)$ \\
\hline $\mathrm{C} 11$ & $13(3)$ & $10(3)$ & $34(4)$ & $-6(3)$ & $4(3)$ & $0(3)$ \\
\hline $\mathrm{C} 12$ & $11(3)$ & $15(4)$ & $20(4)$ & $-6(3)$ & $-2(3)$ & $0(3)$ \\
\hline $\mathrm{C} 13$ & $12(3)$ & $17(4)$ & $15(3)$ & $-1(3)$ & $1(3)$ & $-1(3)$ \\
\hline C14 & $15(3)$ & $23(4)$ & $19(3)$ & $-3(3)$ & $6(3)$ & $-1(3)$ \\
\hline $\mathrm{C} 15$ & $10(3)$ & $23(4)$ & $14(3)$ & $-5(3)$ & $-4(2)$ & $2(3)$ \\
\hline $\mathrm{C} 16$ & $18(3)$ & $18(4)$ & $16(3)$ & $3(3)$ & $3(3)$ & $-1(3)$ \\
\hline $\mathrm{C} 17$ & $17(3)$ & $5(2)$ & $14(3)$ & $-3(2)$ & $-2(2)$ & $0(2)$ \\
\hline $\mathrm{C} 18$ & $34(4)$ & $14(4)$ & 19(4) & 1(3) & $-3(3)$ & $-1(3)$ \\
\hline C19 & $32(4)$ & $19(3)$ & 21(3) & $1(3)$ & $6(3)$ & $-7(3)$ \\
\hline $\mathrm{C} 20$ & $30(4)$ & $14(3)$ & 24(4) & $-10(3)$ & 11(3) & $-11(3)$ \\
\hline $\mathrm{C} 21$ & $24(4)$ & $24(4)$ & $16(3)$ & $-4(3)$ & $-3(3)$ & $-4(3)$ \\
\hline $\mathrm{C} 22$ & $18(3)$ & $7(2)$ & $10(3)$ & $0(2)$ & $3(2)$ & $0(2)$ \\
\hline $\mathrm{C} 23$ & $13(3)$ & $5(3)$ & $17(3)$ & $-2(3)$ & $0(2)$ & $-4(2)$ \\
\hline C24 & 11(3) & $19(4)$ & $5(3)$ & $2(2)$ & $2(2)$ & $5(3)$ \\
\hline $\mathrm{C} 25$ & $14(3)$ & 7(3) & $12(3)$ & $3(2)$ & $0(2)$ & $-6(2)$ \\
\hline $\mathrm{C} 26$ & $9(3)$ & $12(3)$ & $16(3)$ & $-2(3)$ & $1(2)$ & $0(2)$ \\
\hline $\mathrm{C} 27$ & $13(3)$ & $24(4)$ & $34(4)$ & $-11(3)$ & $9(3)$ & $-1(3)$ \\
\hline C28 & $13(3)$ & $20(4)$ & $24(4)$ & $-3(3)$ & $-4(3)$ & $0(3)$ \\
\hline C29 & $19(3)$ & $10(3)$ & 13(3) & $-1(3)$ & $-2(3)$ & $2(3)$ \\
\hline $\mathrm{C} 30$ & $18(3)$ & $15(3)$ & 19(3) & $-6(3)$ & $7(3)$ & $-4(3)$ \\
\hline C31 & $14(3)$ & $25(4)$ & $25(4)$ & $-7(3)$ & $2(3)$ & $4(3)$ \\
\hline
\end{tabular}




\begin{tabular}{|c|c|c|c|c|c|c|}
\hline C32 & $13(3)$ & $19(4)$ & 24(4) & $-4(3)$ & $1(3)$ & $6(3)$ \\
\hline N1 & $14(3)$ & $12(3)$ & $12(3)$ & $2(2)$ & $-3(2)$ & $-1(2)$ \\
\hline $\mathrm{N} 2$ & $18(3)$ & $13(3)$ & $7(2)$ & $-3(2)$ & $1(2)$ & $2(2)$ \\
\hline N3 & $17(3)$ & $11(3)$ & $6(2)$ & $3(2)$ & $-1(2)$ & $2(2)$ \\
\hline N4 & $9(2)$ & $8(3)$ & 11(2) & $-1(2)$ & $-1(2)$ & $-5(2)$ \\
\hline $\mathrm{O} 1$ & $11(2)$ & $15(2)$ & $9(2)$ & $-2(2)$ & $-3(2)$ & $-3(2)$ \\
\hline $\mathrm{O} 2$ & $18(2)$ & $8(2)$ & 11(2) & $7(2)$ & $-4(2)$ & $-3(2)$ \\
\hline $\mathrm{O} 3$ & $17(2)$ & $15(3)$ & $14(2)$ & $-3(2)$ & $-1(2)$ & $-5(2)$ \\
\hline $\mathrm{O} 4$ & $25(3)$ & $17(3)$ & 13(3) & $5(2)$ & $1(2)$ & $-1(2)$ \\
\hline O5 & $12(2)$ & $12(3)$ & $15(2)$ & $-2(2)$ & $2(2)$ & $-2(2)$ \\
\hline O6 & $14(3)$ & $18(3)$ & 21(3) & $-4(2)$ & $3(2)$ & $-4(2)$ \\
\hline $\mathrm{O} 7$ & $24(3)$ & $19(3)$ & $27(3)$ & $-4(3)$ & $0(3)$ & $3(2)$ \\
\hline $\mathrm{O} 8$ & $19(3)$ & $20(3)$ & $26(3)$ & $-2(3)$ & $10(2)$ & $7(2)$ \\
\hline O9 & $16(3)$ & $14(3)$ & $15(3)$ & $0(2)$ & $3(2)$ & $-2(2)$ \\
\hline $\mathrm{O} 10$ & $18(3)$ & $14(3)$ & $37(4)$ & $-6(3)$ & $12(2)$ & $-1(2)$ \\
\hline O11 & $13(2)$ & $31(3)$ & 23(3) & $-1(3)$ & $-5(2)$ & $7(2)$ \\
\hline $\mathrm{O} 12$ & $25(3)$ & $22(3)$ & $30(3)$ & $-8(2)$ & $-5(2)$ & $11(2)$ \\
\hline O13 & 71(4) & $16(3)$ & $30(3)$ & $-9(3)$ & $5(3)$ & $-25(3)$ \\
\hline O14 & $15(2)$ & $9(2)$ & $9(2)$ & $1(2)$ & $-1(2)$ & $-3(2)$ \\
\hline O15 & $15(2)$ & $12(2)$ & $7(2)$ & $0(2)$ & $-1(2)$ & $1(2)$ \\
\hline O16 & $20(3)$ & $12(3)$ & $18(3)$ & $-1(2)$ & $-5(2)$ & $-4(2)$ \\
\hline O17 & $23(3)$ & $9(2)$ & $15(3)$ & $2(2)$ & $6(2)$ & $-3(2)$ \\
\hline O18 & $10(2)$ & $8(3)$ & 17(3) & $-3(2)$ & $3(2)$ & $2(2)$ \\
\hline O19 & $21(3)$ & $13(3)$ & $24(3)$ & $-2(2)$ & $7(2)$ & $-5(2)$ \\
\hline $\mathrm{O} 20$ & $17(3)$ & 11(3) & $27(3)$ & $-3(2)$ & $2(2)$ & $-2(2)$ \\
\hline $\mathrm{O} 21$ & $19(3)$ & $20(3)$ & $26(3)$ & $4(3)$ & $4(2)$ & $4(2)$ \\
\hline $\mathrm{O} 22$ & $10(2)$ & $12(2)$ & $18(3)$ & $-1(2)$ & $1(2)$ & $1(2)$ \\
\hline $\mathrm{O} 23$ & $17(3)$ & $14(3)$ & $37(4)$ & $-3(3)$ & $11(2)$ & $-6(2)$ \\
\hline $\mathrm{O} 24$ & $39(4)$ & $29(3)$ & $23(3)$ & $-4(3)$ & $-16(3)$ & $10(3)$ \\
\hline $\mathrm{O} 25$ & $64(4)$ & $19(3)$ & $54(5)$ & $-8(3)$ & $-24(4)$ & $17(3)$ \\
\hline $\mathrm{O} 26$ & $123(7)$ & $49(5)$ & $27(4)$ & $-12(4)$ & $8(4)$ & $-53(5)$ \\
\hline Pt1 & $10(1)$ & $9(1)$ & $8(1)$ & $0(1)$ & $-1(1)$ & $0(1)$ \\
\hline $\mathrm{Pt} 2$ & $9(1)$ & $7(1)$ & $9(1)$ & $0(1)$ & $0(1)$ & $-1(1)$ \\
\hline
\end{tabular}


Table S5. Hydrogen coordinates ( $\left.x 1^{4}\right)$ and isotropic displacement parameters $\left(\AA^{2} \times 10^{3}\right)$ for 1 .

\begin{tabular}{|c|c|c|c|c|}
\hline & $\mathrm{x}$ & $\mathrm{y}$ & $\mathrm{z}$ & $\mathrm{U}(\mathrm{eq})$ \\
\hline H01B & 2631 & 2471 & 1774 & 16 \\
\hline $\mathrm{H} 2 \mathrm{~A}$ & 4996 & 749 & 1841 & 19 \\
\hline $\mathrm{H} 2 \mathrm{~B}$ & 3937 & 1037 & 2423 & 19 \\
\hline $\mathrm{H} 3 \mathrm{~A}$ & 1911 & 265 & 1838 & 28 \\
\hline H3B & 3174 & -732 & 1851 & 28 \\
\hline $\mathrm{H} 4 \mathrm{~A}$ & 2052 & -530 & 793 & 29 \\
\hline $\mathrm{H} 4 \mathrm{~B}$ & 3778 & -266 & 788 & 29 \\
\hline $\mathrm{H} 5 \mathrm{~A}$ & 2544 & 1259 & 179 & 18 \\
\hline H5B & 1529 & 1562 & 775 & 18 \\
\hline H01P & 4643 & 1847 & 801 & 15 \\
\hline $\mathrm{H} 10 \mathrm{~A}$ & 9432 & 2893 & 793 & 21 \\
\hline H10B & 9273 & 3550 & 107 & 21 \\
\hline H11A & 7309 & 1714 & 528 & 23 \\
\hline H11B & 7138 & 2375 & -156 & 23 \\
\hline $\mathrm{H} 14 \mathrm{~A}$ & 311 & 5691 & 1829 & 22 \\
\hline H14B & 1153 & 5693 & 2520 & 22 \\
\hline $\mathrm{H} 15 \mathrm{~A}$ & -569 & 7316 & 2330 & 19 \\
\hline H15B & 553 & 7849 & 1829 & 19 \\
\hline $\mathrm{H} 01 \mathrm{~S}$ & 4846 & 8255 & 3770 & 15 \\
\hline H18A & 6824 & 8902 & 2778 & 27 \\
\hline H18B & 5136 & 9304 & 2788 & 27 \\
\hline H19A & 5594 & 10416 & 3744 & 29 \\
\hline H19B & 6706 & 10859 & 3211 & 29 \\
\hline $\mathrm{H} 20 \mathrm{~A}$ & 8603 & 9675 & 3683 & 27 \\
\hline $\mathrm{H} 20 \mathrm{~B}$ & 7981 & 10550 & 4222 & 27 \\
\hline $\mathrm{H} 21 \mathrm{~A}$ & 6617 & 8978 & 4653 & 26 \\
\hline $\mathrm{H} 21 \mathrm{~B}$ & 8302 & 8576 & 4632 & 26 \\
\hline $\mathrm{H} 01 \mathrm{~F}$ & 7816 & 7482 & 3664 & 14 \\
\hline $\mathrm{H} 26 \mathrm{~A}$ & 672 & 7283 & 4203 & 15 \\
\hline $\mathrm{H} 26 \mathrm{~B}$ & 685 & 6546 & 4866 & 15 \\
\hline
\end{tabular}




\begin{tabular}{lrrrr} 
H27A & 2786 & 7585 & 5236 & 28 \\
H27B & 2831 & 8296 & 4568 & 28 \\
H30A & 9607 & 4351 & 3115 & 21 \\
H30B & 8759 & 4297 & 2426 & 21 \\
H31A & 10475 & 2656 & 2656 & 26 \\
H31B & 9337 & 2172 & 3165 & 26 \\
H1NA & 5619 & 2828 & 1903 & 15 \\
H1NB & 4466 & 3438 & 2259 & 15 \\
H2NA & 2547 & 3630 & 651 & 15 \\
H2NB & 3820 & 3429 & 244 & 15 \\
H3NA & 5891 & 6796 & 2807 & 14 \\
H3NB & 4336 & 7033 & 2936 & 14 \\
H4NA & 6026 & 6858 & 4668 & 11 \\
H4NB & 7341 & 6214 & 4460 & 11 \\
H8O & 10365 & 988 & -621 & 26 \\
H13A & 1867 & 10453 & 4113 & 58 \\
H13B & 1759 & 9959 & 3466 & 58 \\
H21O & -419 & 9019 & 5569 & 26 \\
H26C & 8045 & -364 & 865 & 99 \\
H26D & 7388 & -1187 & 1326 & 99 \\
& & & & \\
\hline
\end{tabular}


Table S6. Torsion angles $\left[{ }^{\circ}\right]$ for 1.

\begin{tabular}{|c|c|c|c|}
\hline N1-C1-C2-C3 & $-177.5(6)$ & $\mathrm{C} 20-\mathrm{C} 21-\mathrm{C} 22-\mathrm{N} 4$ & $-175.8(5)$ \\
\hline $\mathrm{C} 6-\mathrm{C} 1-\mathrm{C} 2-\mathrm{C} 3$ & $-57.6(7)$ & C20-C21-C22-C17 & $-56.2(8)$ \\
\hline $\mathrm{C} 1-\mathrm{C} 2-\mathrm{C} 3-\mathrm{C} 4$ & $54.6(8)$ & O16-C23-C24-O17 & $-2.9(11)$ \\
\hline $\mathrm{C} 2-\mathrm{C} 3-\mathrm{C} 4-\mathrm{C} 5$ & $-54.5(8)$ & $\mathrm{O} 14-\mathrm{C} 23-\mathrm{C} 24-\mathrm{O} 17$ & $178.1(7)$ \\
\hline C3-C4-C5-C6 & $55.8(8)$ & O16-C23-C24-O15 & $179.6(6)$ \\
\hline $\mathrm{C} 4-\mathrm{C} 5-\mathrm{C} 6-\mathrm{N} 2$ & $177.9(6)$ & $\mathrm{O} 14-\mathrm{C} 23-\mathrm{C} 24-\mathrm{O} 15$ & $0.6(9)$ \\
\hline C4-C5-C6-C1 & $-59.7(7)$ & O19-C25-C26-C27 & $163.2(7)$ \\
\hline N1-C1-C6-N2 & $-50.0(6)$ & $\mathrm{O} 18-\mathrm{C} 25-\mathrm{C} 26-\mathrm{C} 27$ & $-17.1(9)$ \\
\hline $\mathrm{C} 2-\mathrm{C} 1-\mathrm{C} 6-\mathrm{N} 2$ & $-172.6(5)$ & C25-C26-C27-C28 & $177.8(7)$ \\
\hline N1-C1-C6-C5 & $-175.8(5)$ & $\mathrm{C} 26-\mathrm{C} 27-\mathrm{C} 28-\mathrm{O} 20$ & $-125.4(10)$ \\
\hline $\mathrm{C} 2-\mathrm{C} 1-\mathrm{C} 6-\mathrm{C} 5$ & $61.5(7)$ & $\mathrm{C} 26-\mathrm{C} 27-\mathrm{C} 28-\mathrm{O} 21$ & $54.9(10)$ \\
\hline $\mathrm{O} 3-\mathrm{C} 7-\mathrm{C} 8-\mathrm{O} 4$ & $-0.4(11)$ & $\mathrm{O} 23-\mathrm{C} 29-\mathrm{C} 30-\mathrm{C} 31$ & $-11.9(11)$ \\
\hline $\mathrm{O} 1-\mathrm{C} 7-\mathrm{C} 8-\mathrm{O} 4$ & $178.0(7)$ & O22-C29-C30-C31 & $169.1(7)$ \\
\hline $\mathrm{O} 3-\mathrm{C} 7-\mathrm{C} 8-\mathrm{O} 2$ & $-177.3(7)$ & C29-C30-C31-C32 & $74.4(9)$ \\
\hline $\mathrm{O} 1-\mathrm{C} 7-\mathrm{C} 8-\mathrm{O} 2$ & $1.0(10)$ & $\mathrm{C} 30-\mathrm{C} 31-\mathrm{C} 32-\mathrm{O} 24$ & $3.0(12)$ \\
\hline O6-C9-C10-C11 & $-169.7(7)$ & $\mathrm{C} 30-\mathrm{C} 31-\mathrm{C} 32-\mathrm{O} 25$ & $-176.9(8)$ \\
\hline O5-C9-C10-C11 & $11.4(10)$ & C6-C1-N1-Pt1 & $35.9(6)$ \\
\hline C9-C10-C11-C12 & $179.6(7)$ & C2-C1-N1-Pt1 & $157.5(5)$ \\
\hline C10-C11-C12-O7 & $134.3(9)$ & C5-C6-N2-Pt1 & $164.4(4)$ \\
\hline $\mathrm{C} 10-\mathrm{C} 11-\mathrm{C} 12-\mathrm{O} 8$ & $-47.3(10)$ & C1-C6-N2-Pt1 & $40.6(6)$ \\
\hline O10-C13-C14-C15 & $8.5(12)$ & C22-C17-N3-Pt2 & $44.6(6)$ \\
\hline O9-C13-C14-C15 & $-172.7(7)$ & C18-C17-N3-Pt2 & $168.9(5)$ \\
\hline C13-C14-C15-C16 & $-73.6(9)$ & C17-C22-N4-Pt2 & $36.3(5)$ \\
\hline C14-C15-C16-O11 & $3.6(12)$ & C21-C22-N4-Pt2 & $157.9(5)$ \\
\hline C14-C15-C16-O12 & $-173.4(7)$ & O3-C7-O1-Pt1 & $177.3(6)$ \\
\hline N3-C17-C18-C19 & $179.9(6)$ & C8-C7-O1-Pt1 & $-1.0(8)$ \\
\hline C22-C17-C18-C19 & $-58.2(8)$ & O4-C8-O2-Pt1 & $-177.3(6)$ \\
\hline C17-C18-C19-C20 & $56.3(8)$ & $\mathrm{C} 7-\mathrm{C} 8-\mathrm{O} 2-\mathrm{Pt} 1$ & $-0.5(8)$ \\
\hline C18-C19-C20-C21 & $-57.3(8)$ & O6-C9-O5-Pt1 & $-5.2(11)$ \\
\hline C19-C20-C21-C22 & $56.3(8)$ & C10-C9-O5-Pt1 & $173.7(5)$ \\
\hline N3-C17-C22-N4 & $-53.2(6)$ & O10-C13-O9-Pt1 & $3.6(12)$ \\
\hline C18-C17-C22-N4 & $-178.5(5)$ & C14-C13-O9-Pt1 & $-175.1(4)$ \\
\hline N3-C17-C22-C21 & $-176.6(6)$ & $\mathrm{O} 16-\mathrm{C} 23-\mathrm{O} 14-\mathrm{Pt} 2$ & $-178.8(6)$ \\
\hline C18-C17-C22-C21 & $58.2(7)$ & C24-C23-O14-Pt2 & $0.2(8)$ \\
\hline
\end{tabular}




$\begin{array}{lccc}\text { O17-C24-O15-Pt2 } & -178.4(6) & \text { C26-C25-O18-Pt2 } & -172.8(4) \\ \text { C23-C24-O15-Pt2 } & -1.0(7) & \text { O23-C29-O22-Pt2 } & -3.1(11) \\ \text { O19-C25-O18-Pt2 } & 6.9(11) & \text { C30-C29-O22-Pt2 } & 175.8(5)\end{array}$


Table S7. Hydrogen bonds for 1 [ $\AA$ and ${ }^{\circ}$.

\begin{tabular}{|c|c|c|c|c|}
\hline D-H...A & $d(D-H)$ & $\mathrm{d}(\mathrm{H} \ldots \mathrm{A})$ & $\mathrm{d}(\mathrm{D} \ldots \mathrm{A})$ & $<(\mathrm{DHA})$ \\
\hline C2-H2B...O16 & 0.99 & 2.55 & $3.292(10)$ & 132.0 \\
\hline C5-H5A...O6\#1 & 0.99 & 2.65 & $3.532(10)$ & 148.0 \\
\hline C10-H10B...O7\#2 & 0.99 & 2.54 & $3.342(10)$ & 137.6 \\
\hline C11-H11B...O2\#1 & 0.99 & 2.59 & $3.195(9)$ & 119.6 \\
\hline C15-H15A...O3\#3 & 0.99 & 2.51 & $3.364(8)$ & 144.6 \\
\hline C18-H18A...O3 & 0.99 & 2.53 & $3.246(10)$ & 128.9 \\
\hline C21-H21A...O15\#4 & 0.99 & 2.64 & $3.610(10)$ & 166.0 \\
\hline C21-H21B...O21\#5 & 0.99 & 2.59 & $3.547(9)$ & 162.9 \\
\hline C26-H26B...O20\#6 & 0.99 & 2.59 & $3.428(9)$ & 142.4 \\
\hline C31-H31A...O16\#5 & 0.99 & 2.52 & $3.362(9)$ & 143.1 \\
\hline N1-H1NA...O24 & 0.91 & 1.93 & $2.834(9)$ & 169.5 \\
\hline N1-H1NB...O14 & 0.91 & 2.25 & $3.152(8)$ & 169.0 \\
\hline N1-H1NB...O16 & 0.91 & 2.49 & $3.203(8)$ & 135.5 \\
\hline N2-H2NA...O7\#7 & 0.91 & 2.34 & $3.006(9)$ & 129.5 \\
\hline N2-H2NB...O4\#1 & 0.91 & 1.95 & $2.802(8)$ & 154.7 \\
\hline N3-H3NA...O1 & 0.91 & 2.43 & $3.147(8)$ & 135.9 \\
\hline N3-H3NA...O3 & 0.91 & 2.29 & $3.095(8)$ & 148.1 \\
\hline N3-H3NB...O11 & 0.91 & 1.96 & $2.751(8)$ & 144.6 \\
\hline N4-H4NA...O17\#4 & 0.91 & 1.99 & $2.835(8)$ & 154.6 \\
\hline N4-H4NB...O20\#8 & 0.91 & 2.07 & $2.938(8)$ & 159.4 \\
\hline O8-H8O...О6\#9 & 0.88 & 1.78 & $2.635(8)$ & 165.2 \\
\hline O13-H13А...O20 & 0.90 & 2.05 & $2.899(9)$ & 157.4 \\
\hline O13-H13В...O12 & 0.84 & 1.78 & $2.622(8)$ & 179.8 \\
\hline O21-H21O...O19\#10 & 0.85 & 1.81 & $2.623(8)$ & 158.0 \\
\hline O26-H26C...O7 & 0.90 & 1.98 & $2.871(10)$ & 169.5 \\
\hline O26-H26D...O3\#11 & 0.93 & 1.99 & $2.846(11)$ & 152.2 \\
\hline O26-H26D...O4\#11 & 0.93 & 2.35 & $3.011(9)$ & 127.9 \\
\hline C2-H2B...O16 & 0.99 & 2.55 & $3.292(10)$ & 132.0 \\
\hline C5-H5A...O6\#1 & 0.99 & 2.65 & $3.532(10)$ & 148.0 \\
\hline C10-H10B...O7\#2 & 0.99 & 2.54 & $3.342(10)$ & 137.6 \\
\hline C11-H11B...O2\#1 & 0.99 & 2.59 & $3.195(9)$ & 119.6 \\
\hline C15-H15A...O3\#3 & 0.99 & 2.51 & $3.364(8)$ & 144.6 \\
\hline
\end{tabular}




\begin{tabular}{lllll} 
C18-H18A...O3 & 0.99 & 2.53 & $3.246(10)$ & 128.9 \\
C21-H21A...O15\#4 & 0.99 & 2.64 & $3.610(10)$ & 166.0 \\
C21-H21B...O21\#5 & 0.99 & 2.59 & $3.547(9)$ & 162.9 \\
C26-H26B...O20\#6 & 0.99 & 2.59 & $3.428(9)$ & 142.4 \\
C31-H31A...O16\#5 & 0.99 & 2.52 & $3.362(9)$ & 143.1 \\
N1-H1NA...O24 & 0.91 & 1.93 & $2.834(9)$ & 169.5 \\
N1-H1NB...O14 & 0.91 & 2.25 & $3.152(8)$ & 169.0 \\
N1-H1NB...O16 & 0.91 & 2.49 & $3.203(8)$ & 135.5 \\
N2-H2NA...O7\#7 & 0.91 & 2.34 & $3.006(9)$ & 129.5 \\
N2-H2NB...O4\#1 & 0.91 & 1.95 & $2.802(8)$ & 154.7 \\
N3-H3NA...O1 & 0.91 & 2.43 & $3.147(8)$ & 135.9 \\
N3-H3NA...O3 & 0.91 & 2.29 & $3.095(8)$ & 148.1 \\
N3-H3NB...O11 & 0.91 & 1.96 & $2.751(8)$ & 144.6 \\
N4-H4NA...O17\#4 & 0.91 & 1.99 & $2.835(8)$ & 154.6 \\
N4-H4NB...O20\#8 & 0.91 & 2.07 & $2.938(8)$ & 159.4 \\
O8-H8O...O6\#9 & 0.88 & 1.78 & $2.635(8)$ & 165.2 \\
O13-H13A...O20 & 0.90 & 2.05 & $2.899(9)$ & 157.4 \\
O13-H13B...O12 & 0.84 & 1.78 & $2.622(8)$ & 179.8 \\
O21-H21O...O19\#10 & 0.85 & 1.81 & $2.623(8)$ & 158.0 \\
O26-H26C...O7 & 0.90 & 1.98 & $2.871(10)$ & 169.5 \\
O26-H26D...O3\#11 & 0.93 & 1.99 & $2.846(11)$ & 152.2 \\
O26-H26D...O4\#11 & 0.93 & 2.35 & $3.011(9)$ & 127.9 \\
& & & & \\
\hline
\end{tabular}

Symmetry transformations used to generate equivalent atoms:

$\# 1-\mathrm{x}+1, \mathrm{y}-1 / 2,-\mathrm{z} \quad \# 2-\mathrm{x}+2, \mathrm{y}+1 / 2,-\mathrm{z} \quad \# 3 \mathrm{x}-1, \mathrm{y}, \mathrm{z}$

$\# 4-\mathrm{x}+1, \mathrm{y}+1 / 2,-\mathrm{z}+1 \quad \# 5 \mathrm{x}+1, \mathrm{y}, \mathrm{z} \quad \# 6-\mathrm{x}, \mathrm{y}-1 / 2,-\mathrm{z}+1$

$\# 7-x+1, y+1 / 2,-z \quad \# 8-x+1, y-1 / 2,-z+1 \quad \# 9-x+2, y-1 / 2,-z$

$\# 10-\mathrm{x}, \mathrm{y}+1 / 2,-\mathrm{z}+1 \quad \# 11 \mathrm{x}, \mathrm{y}-1, \mathrm{z}$ 
X-ray Experimental for complex (2) $2\left(\mathrm{C}_{17} \mathrm{H}_{19} \mathrm{~N}_{2} \mathrm{O}_{4} \mathrm{Fe}\right) \mathrm{Pt}\left(\mathrm{C}_{2} \mathrm{O}_{4}\right)\left(\mathrm{C}_{6} \mathrm{H}_{14} \mathrm{~N}_{2}\right) 3 \mathrm{H}_{2} \mathrm{O}$ : Crystals grew as very thin yellow laths by slow diffusion of ether vapor into a solution of 2 in methanol. The data crystal had approximate dimensions; $0.23 \times 0.023 \times 0.012 \mathrm{~mm}$. The data were collected on an Agilent Technologies SuperNova Dual Source diffractometer using a $\mu$-focus $\mathrm{Cu} K \alpha$ radiation source $(\lambda=1.5418 \AA)$ with collimating mirror monochromators. A total of 579 frames of data were collected using $\omega$-scans with a scan range of $1^{\circ}$ and a counting time of 23 seconds per frame with a detector offset of $+/-40.8^{\circ}$ and 75 seconds per frame with a detector offset of $+/-108.3^{\circ}$. The data were collected at $100 \mathrm{~K}$ using an Oxford Cryostream low temperature device. Details of crystal data, data collection and structure refinement are listed in Table 1. Data collection, unit cell refinement and data reduction were performed using Agilent Technologies CrysAlisPro V 1.171.37.31. ${ }^{\mathrm{S} 5}$ The structure was solved by direct methods using SuperFlip ${ }^{\mathrm{S15}}$ and refined by full-matrix least-squares on $\mathrm{F}^{2}$ with anisotropic displacement parameters for the non- $\mathrm{H}$ atoms using SHELXL-2013. ${ }^{\text {S16 }}$ Structure analysis was aided by use of the programs PLATON98 ${ }^{\mathrm{s} 8}$ and WinGX.99 The hydrogen atoms were calculated in ideal positions with isotropic displacement parameters set to 1.2xUeq of the attached atom. The hydrogen atoms on the water molecules could not be located in a $\Delta \mathrm{F}$ map and were not included in the final refinement model.

The function, $\Sigma \mathrm{w}\left(\left|\mathrm{F}_{\mathrm{O}}\right|^{2}-\left|\mathrm{F}_{\mathrm{C}}\right|^{2}\right)^{2}$, was minimized, where $\mathrm{w}=1 /\left[\left(\sigma\left(\mathrm{F}_{\mathrm{O}}\right)\right)^{2}+(0.0427 * \mathrm{P})^{2}+(3.1206 * \mathrm{P})\right]$ and $\mathrm{P}=\left(\left|\mathrm{F}_{\mathrm{O}}\right|^{2}+2\left|\mathrm{~F}_{\mathrm{C}}\right|^{2}\right) / 3 . \mathrm{R}_{\mathrm{W}}\left(\mathrm{F}^{2}\right)$ refined to 0.252 , with $\mathrm{R}(\mathrm{F})$ equal to 0.105 and a goodness of fit, $\mathrm{S},=1.18$. Definitions used for calculating $R(F), R_{W}\left(F^{2}\right)$ and the goodness of fit, $S$, are given below. ${ }^{S 12}$ The data were checked for secondary extinction effects but no correction was necessary. Neutral atom scattering factors and values used to calculate the linear absorption coefficient are from the International Tables for X-ray Crystallography (1992). ${ }^{S 13}$ All figures were generated using SHELXTL/PC. ${ }^{S 14}$ Tables of positional and thermal parameters, bond lengths and angles, torsion angles and figures may be found in the .cif documents that are archived with the Cambridge Crystallographic Data Centre. 
Table S8. Crystal data and structure refinement for 2

Empirical formula

Formula weight

Temperature

Wavelength

Crystal system

Space group

Unit cell dimensions

Volume

Z

Density (calculated)

Absorption coefficient

$\mathrm{F}(000)$

Crystal size

Theta range for data collection

Index ranges

Reflections collected

Independent reflections

Completeness to theta $=67.684^{\circ}$

Absorption correction

Max. and min. transmission

Refinement method

Data / restraints / parameters

Goodness-of-fit on $\mathrm{F}^{2}$

Final R indices [I $>2 \operatorname{sigma}(\mathrm{I})]$

$\mathrm{R}$ indices (all data)

Absolute structure parameter

Extinction coefficient

Largest diff. peak and hole
C42 H58 Fe2 N6 O15 Pt

1193.73

100(2) K

$1.54184 \AA$

orthorhombic

P 212121

$\mathrm{a}=9.8035(3) \AA \quad \alpha=90^{\circ}$.

$\mathrm{b}=10.7651(6) \AA \quad \beta=90^{\circ}$.

$\mathrm{c}=43.577(2) \AA \quad \gamma=90^{\circ}$.

4598.9(4) $\AA^{3}$

4

$1.724 \mathrm{Mg} / \mathrm{m}^{3}$

$11.204 \mathrm{~mm}^{-1}$

2408

$0.230 \times 0.023 \times 0.012 \mathrm{~mm}$

4.058 to $74.319^{\circ}$.

$-12<=\mathrm{h}<=8,-5<=\mathrm{k}<=13,-51<=\mathrm{l}<=54$

15720

$8977[\mathrm{R}(\mathrm{int})=0.1294]$

$99.9 \%$

Semi-empirical from equivalents

1.00 and 0.478

Full-matrix least-squares on $\mathrm{F}^{2}$

8977 / 684 / 592

1.223

$\mathrm{R} 1=0.1051, \mathrm{wR} 2=0.2136$

$\mathrm{R} 1=0.1560, \mathrm{wR} 2=0.2517$

$-0.002(13)$

$\mathrm{n} / \mathrm{a}$

5.239 and -3.860 e. $\AA^{-3}$ 
Table S9. Atomic coordinates $\left(x 0^{4}\right)$ and equivalent isotropic displacement parameters $\left(\AA^{2} \times 10^{3}\right)$ for 2. $U(e q)$ is defined as one third of the trace of the orthogonalized $U^{i j}$ tensor.

\begin{tabular}{|c|c|c|c|c|}
\hline & $\mathrm{x}$ & $\mathrm{y}$ & $\mathrm{z}$ & $\mathrm{U}(\mathrm{eq})$ \\
\hline $\mathrm{C} 1$ & $6160(30)$ & $4360(30)$ & $4553(6)$ & $30(6)$ \\
\hline $\mathrm{C} 2$ & $6170(30)$ & $3680(30)$ & $4239(6)$ & $42(7)$ \\
\hline $\mathrm{C} 3$ & $7320(30)$ & $4190(20)$ & $4027(6)$ & $33(5)$ \\
\hline $\mathrm{C} 4$ & $7050(30)$ & $5440(30)$ & $3897(6)$ & $31(5)$ \\
\hline $\mathrm{C} 5$ & $8080(20)$ & $7270(20)$ & $3647(6)$ & $24(5)$ \\
\hline C6 & $9230(30)$ & $7370(20)$ & $3425(6)$ & $23(5)$ \\
\hline $\mathrm{C} 7$ & $10350(30)$ & $5930(20)$ & $3055(6)$ & $26(5)$ \\
\hline $\mathrm{C} 8$ & $10170(30)$ & $5010(30)$ & $2818(6)$ & $29(4)$ \\
\hline $\mathrm{C} 9$ & $8960(30)$ & $4710(30)$ & $2642(6)$ & $32(4)$ \\
\hline $\mathrm{C} 10$ & $9310(30)$ & $3720(30)$ & 2441(5) & $31(4)$ \\
\hline C11 & $10610(30)$ & $3340(30)$ & $2501(6)$ & $30(4)$ \\
\hline $\mathrm{C} 12$ & $11220(30)$ & $4150(20)$ & $2730(6)$ & $30(4)$ \\
\hline $\mathrm{C} 13$ & $9340(30)$ & $3020(30)$ & $3365(6)$ & $35(5)$ \\
\hline $\mathrm{C} 14$ & $7980(30)$ & $2970(30)$ & $3218(6)$ & $38(5)$ \\
\hline $\mathrm{C} 15$ & $8110(30)$ & $1950(30)$ & $3005(6)$ & $36(5)$ \\
\hline $\mathrm{C} 16$ & $9370(30)$ & $1390(30)$ & $3024(6)$ & $37(5)$ \\
\hline $\mathrm{C} 17$ & $10140(30)$ & $2050(30)$ & $3242(6)$ & $38(5)$ \\
\hline $\mathrm{C} 18$ & $7330(40)$ & $8760(30)$ & $5242(6)$ & $48(8)$ \\
\hline C19 & $7120(50)$ & $9530(30)$ & $5523(6)$ & $50(9)$ \\
\hline $\mathrm{C} 20$ & $7330(30)$ & $8760(30)$ & $5825(5)$ & $37(6)$ \\
\hline $\mathrm{C} 21$ & $7000(30)$ & $9640(20)$ & 6096(6) & $30(5)$ \\
\hline $\mathrm{C} 22$ & $7990(30)$ & $10890(20)$ & $6508(5)$ & $26(5)$ \\
\hline $\mathrm{C} 23$ & $9180(30)$ & $10770(20)$ & $6727(6)$ & $26(5)$ \\
\hline $\mathrm{C} 42$ & $8090(40)$ & $7850(30)$ & $4493(7)$ & $38(5)$ \\
\hline $\mathrm{C} 41$ & $6500(40)$ & $7980(30)$ & $4490(7)$ & $42(6)$ \\
\hline $\mathrm{C} 24$ & $10340(30)$ & $8990(20)$ & $6949(5)$ & $23(4)$ \\
\hline $\mathrm{C} 25$ & $10260(30)$ & $7810(30)$ & 7111(7) & $33(4)$ \\
\hline $\mathrm{C} 26$ & $9180(30)$ & $7410(30)$ & $7299(6)$ & $34(5)$ \\
\hline $\mathrm{C} 27$ & $9420(30)$ & $6250(40)$ & 7444(7) & $41(5)$ \\
\hline $\mathrm{C} 28$ & $10750(30)$ & $5900(30)$ & $7337(7)$ & $43(5)$ \\
\hline C29 & $11240(30)$ & $6870(30)$ & $7139(7)$ & $37(5)$ \\
\hline
\end{tabular}




\begin{tabular}{|c|c|c|c|c|}
\hline $\mathrm{C} 30$ & $8930(40)$ & $6310(40)$ & $6525(7)$ & $54(6)$ \\
\hline $\mathrm{C} 31$ & $7820(40)$ & $5850(30)$ & $6710(7)$ & $51(5)$ \\
\hline $\mathrm{C} 32$ & $8200(40)$ & $4800(30)$ & $6830(8)$ & $50(6)$ \\
\hline $\mathrm{C} 33$ & $9580(40)$ & $4510(30)$ & $6735(8)$ & $55(6)$ \\
\hline C34 & $10120(40)$ & $5420(30)$ & 6532(9) & $60(6)$ \\
\hline C35 & $6510(30)$ & $4480(30)$ & $5400(6)$ & $36(5)$ \\
\hline $\mathrm{C} 36$ & $7980(30)$ & $4930(30)$ & $5491(6)$ & $35(5)$ \\
\hline C37 & $8750(40)$ & $3920(30)$ & $5659(7)$ & $49(7)$ \\
\hline C38 & $8070(40)$ & $3470(30)$ & $5941(6)$ & $52(7)$ \\
\hline C39 & $6670(40)$ & $3000(30)$ & $5847(8)$ & $56(8)$ \\
\hline $\mathrm{C} 40$ & $5780(40)$ & $4000(30)$ & $5685(6)$ & $43(7)$ \\
\hline $\mathrm{Fe} 2$ & $9568(5)$ & $6124(4)$ & $6967(1)$ & $33(1)$ \\
\hline $\mathrm{Fe} 3$ & $9517(5)$ & $3224(4)$ & $2898(1)$ & $28(1)$ \\
\hline N1 & $8140(20)$ & $6110(20)$ & $3810(5)$ & $29(5)$ \\
\hline $\mathrm{N} 2$ & $9190(20)$ & $6420(19)$ & $3183(4)$ & 19(4) \\
\hline N3 & $8140(20)$ & $10040(20)$ & $6245(5)$ & $28(5)$ \\
\hline N4 & $9170(20)$ & $9540(20)$ & $6869(5)$ & $29(5)$ \\
\hline N5 & $5850(30)$ & $5460(20)$ & $5227(5)$ & $32(4)$ \\
\hline N6 & $8640(30)$ & $5370(20)$ & $5200(5)$ & $30(4)$ \\
\hline $\mathrm{O} 1$ & $7210(20)$ & 4992(15) & $4629(4)$ & $30(4)$ \\
\hline $\mathrm{O} 2$ & $5130(30)$ & $4240(20)$ & $4714(5)$ & $64(8)$ \\
\hline $\mathrm{O} 3$ & $5880(20)$ & $5890(20)$ & $3871(5)$ & $44(5)$ \\
\hline $\mathrm{O} 4$ & $11498(18)$ & $6408(19)$ & $3119(4)$ & $31(4)$ \\
\hline $\mathrm{O} 5$ & $7254(19)$ & $7604(14)$ & $5284(3)$ & $21(3)$ \\
\hline O6 & $7450(30)$ & $9289(17)$ & 4990(4) & $67(8)$ \\
\hline $\mathrm{O} 7$ & $5840(20)$ & $10000(20)$ & $6158(5)$ & $38(5)$ \\
\hline $\mathrm{O} 8$ & $11515(19)$ & $9438(18)$ & $6887(4)$ & $30(4)$ \\
\hline O9 & $5940(20)$ & $7281(18)$ & $4695(5)$ & $39(4)$ \\
\hline $\mathrm{O} 10$ & $8670(20)$ & $7151(18)$ & $4686(4)$ & $38(4)$ \\
\hline O11 & $5980(30)$ & $8660(20)$ & $4320(5)$ & $59(6)$ \\
\hline $\mathrm{O} 12$ & $8790(30)$ & $8530(20)$ & $4313(5)$ & $49(5)$ \\
\hline $\mathrm{O} 13$ & $2590(20)$ & $3340(19)$ & $5227(4)$ & $72(8)$ \\
\hline O14 & $4680(20)$ & 1733(19) & $5105(4)$ & $70(8)$ \\
\hline $\mathrm{O} 15$ & $2330(20)$ & $4160(20)$ & $5875(5)$ & $86(10)$ \\
\hline Pt1 & $7239(2)$ & $6316(1)$ & 4949(1) & $37(1)$ \\
\hline
\end{tabular}


Table S10. Bond lengths $[\AA \AA]$ and angles $\left[^{\circ}\right]$ for 2

\begin{tabular}{|c|c|c|c|}
\hline $\mathrm{C} 1-\mathrm{O} 2$ & $1.24(4)$ & $\mathrm{C} 12-\mathrm{H} 12$ & 0.95 \\
\hline $\mathrm{C} 1-\mathrm{O} 1$ & $1.28(4)$ & C13-C17 & $1.42(4)$ \\
\hline $\mathrm{C} 1-\mathrm{C} 2$ & $1.55(4)$ & C13-C14 & $1.48(4)$ \\
\hline $\mathrm{C} 2-\mathrm{C} 3$ & $1.56(4)$ & $\mathrm{C} 13-\mathrm{Fe} 3$ & $2.06(3)$ \\
\hline $\mathrm{C} 2-\mathrm{H} 2 \mathrm{~A}$ & 0.99 & C13-H13 & 0.95 \\
\hline $\mathrm{C} 2-\mathrm{H} 2 \mathrm{~B}$ & 0.99 & C14-C15 & $1.45(4)$ \\
\hline $\mathrm{C} 3-\mathrm{C} 4$ & $1.49(4)$ & $\mathrm{C} 14-\mathrm{Fe} 3$ & $2.07(3)$ \\
\hline C3-H3A & 0.99 & C14-H14 & 0.95 \\
\hline C3-H3B & 0.99 & C15-C16 & $1.38(4)$ \\
\hline $\mathrm{C} 4-\mathrm{O} 3$ & $1.24(3)$ & $\mathrm{C} 15-\mathrm{Fe} 3$ & $2.00(3)$ \\
\hline C4-N1 & $1.35(3)$ & C15-H15 & 0.95 \\
\hline $\mathrm{C} 5-\mathrm{N} 1$ & $1.44(3)$ & $\mathrm{C} 16-\mathrm{C} 17$ & $1.41(4)$ \\
\hline $\mathrm{C} 5-\mathrm{C} 6$ & $1.49(3)$ & $\mathrm{C} 16-\mathrm{Fe} 3$ & $2.05(3)$ \\
\hline C5-H5A & 0.99 & C16-H16 & 0.95 \\
\hline C5-H5B & 0.99 & $\mathrm{C} 17-\mathrm{Fe} 3$ & $2.06(3)$ \\
\hline C6-N2 & $1.47(3)$ & C17-H17 & 0.95 \\
\hline C6-H6A & 0.99 & C18-O6 & $1.24(3)$ \\
\hline C6-H6B & 0.99 & C18-O5 & $1.26(3)$ \\
\hline C7-O4 & $1.27(3)$ & C18-C19 & $1.49(4)$ \\
\hline C7-N2 & $1.38(3)$ & C19-C20 & $1.57(3)$ \\
\hline $\mathrm{C} 7-\mathrm{C} 8$ & $1.44(3)$ & C19-H19A & 0.99 \\
\hline C8-C12 & $1.44(4)$ & C19-H19B & 0.99 \\
\hline C8-C9 & $1.45(4)$ & $\mathrm{C} 20-\mathrm{C} 21$ & $1.55(3)$ \\
\hline $\mathrm{C} 8-\mathrm{Fe} 3$ & $2.05(3)$ & $\mathrm{C} 20-\mathrm{H} 20 \mathrm{~A}$ & 0.99 \\
\hline C9-C10 & $1.42(4)$ & $\mathrm{C} 20-\mathrm{H} 20 \mathrm{~B}$ & 0.99 \\
\hline $\mathrm{C} 9-\mathrm{Fe} 3$ & $2.03(3)$ & $\mathrm{C} 21-\mathrm{O} 7$ & $1.23(3)$ \\
\hline C9-H9 & 0.95 & $\mathrm{C} 21-\mathrm{N} 3$ & $1.36(3)$ \\
\hline C10-C11 & $1.36(4)$ & C22-N3 & $1.48(3)$ \\
\hline $\mathrm{C} 10-\mathrm{Fe} 3$ & $2.07(2)$ & $\mathrm{C} 22-\mathrm{C} 23$ & $1.51(3)$ \\
\hline C10-H10 & 0.95 & $\mathrm{C} 22-\mathrm{H} 22 \mathrm{~A}$ & 0.99 \\
\hline $\mathrm{C} 11-\mathrm{C} 12$ & $1.45(3)$ & $\mathrm{C} 22-\mathrm{H} 22 \mathrm{~B}$ & 0.99 \\
\hline $\mathrm{C} 11-\mathrm{Fe} 3$ & $2.04(3)$ & C23-N4 & $1.46(3)$ \\
\hline C11-H11 & 0.95 & $\mathrm{C} 23-\mathrm{H} 23 \mathrm{~A}$ & 0.99 \\
\hline $\mathrm{C} 12-\mathrm{Fe} 3$ & $2.08(3)$ & $\mathrm{C} 23-\mathrm{H} 23 \mathrm{~B}$ & 0.99 \\
\hline
\end{tabular}




\begin{tabular}{|c|c|c|c|}
\hline $\mathrm{C} 42-\mathrm{O} 10$ & $1.26(3)$ & $\mathrm{C} 34-\mathrm{Fe} 2$ & $2.11(4)$ \\
\hline $\mathrm{C} 42-\mathrm{O} 12$ & $1.27(4)$ & C34-H34 & 0.95 \\
\hline C42-C41 & $1.56(5)$ & C35-N5 & $1.45(4)$ \\
\hline C41-O11 & $1.16(4)$ & $\mathrm{C} 35-\mathrm{C} 40$ & $1.52(4)$ \\
\hline C41-O9 & $1.29(4)$ & C35-C36 & $1.57(4)$ \\
\hline C24-O8 & $1.28(3)$ & C35-H35 & 1.00 \\
\hline C24-N4 & $1.34(3)$ & C36-N6 & $1.50(3)$ \\
\hline $\mathrm{C} 24-\mathrm{C} 25$ & $1.45(4)$ & C36-C37 & $1.51(4)$ \\
\hline C25-C29 & $1.40(4)$ & C36-H36 & 1.00 \\
\hline $\mathrm{C} 25-\mathrm{C} 26$ & $1.41(4)$ & C37-C38 & $1.48(4)$ \\
\hline $\mathrm{C} 25-\mathrm{Fe} 2$ & $2.04(3)$ & C37-H37A & 0.99 \\
\hline $\mathrm{C} 26-\mathrm{C} 27$ & $1.42(4)$ & C37-H37B & 0.99 \\
\hline $\mathrm{C} 26-\mathrm{Fe} 2$ & $2.04(3)$ & C38-C39 & $1.52(5)$ \\
\hline $\mathrm{C} 26-\mathrm{H} 26$ & 0.95 & C38-H38A & 0.99 \\
\hline C27-C28 & $1.44(4)$ & C38-H38B & 0.99 \\
\hline $\mathrm{C} 27-\mathrm{Fe} 2$ & $2.09(3)$ & C39-C40 & $1.55(4)$ \\
\hline $\mathrm{C} 27-\mathrm{H} 27$ & 0.95 & C39-H39A & 0.99 \\
\hline C28-C29 & $1.43(4)$ & C39-H39B & 0.99 \\
\hline $\mathrm{C} 28-\mathrm{Fe} 2$ & $2.00(3)$ & $\mathrm{C} 40-\mathrm{H} 40 \mathrm{~A}$ & 0.99 \\
\hline C28-H28 & 0.95 & $\mathrm{C} 40-\mathrm{H} 40 \mathrm{~B}$ & 0.99 \\
\hline $\mathrm{C} 29-\mathrm{Fe} 2$ & $1.97(3)$ & $\mathrm{N} 1-\mathrm{H} 1$ & 0.88 \\
\hline C29-H29 & 0.95 & $\mathrm{~N} 2-\mathrm{H} 2$ & 0.88 \\
\hline C30-C31 & $1.44(5)$ & N3-H3 & 0.88 \\
\hline C30-C34 & $1.51(5)$ & N4-H4 & 0.88 \\
\hline $\mathrm{C} 30-\mathrm{Fe} 2$ & $2.04(3)$ & N5-Pt1 & $2.04(2)$ \\
\hline C30-H30 & 0.95 & N5-H5C & 0.99 \\
\hline C31-C32 & $1.31(4)$ & N5-H5D & 0.99 \\
\hline $\mathrm{C} 31-\mathrm{Fe} 2$ & $2.07(3)$ & N6-Pt1 & $2.03(2)$ \\
\hline C31-H31 & 0.95 & N6-H6C & 0.99 \\
\hline C32-C33 & $1.45(5)$ & N6-H6D & 0.99 \\
\hline $\mathrm{C} 32-\mathrm{Fe} 2$ & $2.05(3)$ & O1-Pt1 & $1.996(16)$ \\
\hline C32-H32 & 0.95 & O5-Pt1 & $2.012(15)$ \\
\hline C33-C34 & $1.43(5)$ & O9-Pt1 & $1.99(2)$ \\
\hline $\mathrm{C} 33-\mathrm{Fe} 2$ & $2.01(3)$ & O10-Pt1 & $2.02(2)$ \\
\hline C33-H33 & 0.95 & & \\
\hline
\end{tabular}




\begin{tabular}{|c|c|c|c|}
\hline $\mathrm{O} 2-\mathrm{C} 1-\mathrm{O} 1$ & $125(3)$ & $\mathrm{C} 12-\mathrm{C} 8-\mathrm{Fe} 3$ & $70.6(16)$ \\
\hline $\mathrm{O} 2-\mathrm{C} 1-\mathrm{C} 2$ & $117(3)$ & $\mathrm{C} 7-\mathrm{C} 8-\mathrm{Fe} 3$ & $124.0(19)$ \\
\hline $\mathrm{O} 1-\mathrm{C} 1-\mathrm{C} 2$ & $118(2)$ & $\mathrm{C} 9-\mathrm{C} 8-\mathrm{Fe} 3$ & $68.3(16)$ \\
\hline $\mathrm{C} 1-\mathrm{C} 2-\mathrm{C} 3$ & $111(3)$ & C10-C9-C8 & $107(3)$ \\
\hline $\mathrm{C} 1-\mathrm{C} 2-\mathrm{H} 2 \mathrm{~A}$ & 109.4 & $\mathrm{C} 10-\mathrm{C} 9-\mathrm{Fe} 3$ & 71.4(17) \\
\hline $\mathrm{C} 3-\mathrm{C} 2-\mathrm{H} 2 \mathrm{~A}$ & 109.4 & $\mathrm{C} 8-\mathrm{C} 9-\mathrm{Fe} 3$ & 70.1(17) \\
\hline C1-C2-H2B & 109.4 & C10-C9-H9 & 126.5 \\
\hline $\mathrm{C} 3-\mathrm{C} 2-\mathrm{H} 2 \mathrm{~B}$ & 109.4 & C8-C9-H9 & 126.5 \\
\hline $\mathrm{H} 2 \mathrm{~A}-\mathrm{C} 2-\mathrm{H} 2 \mathrm{~B}$ & 108.0 & Fe3-C9-H9 & 123.7 \\
\hline $\mathrm{C} 4-\mathrm{C} 3-\mathrm{C} 2$ & $115(3)$ & C11-C10-C9 & $109(3)$ \\
\hline C4-C3-H3A & 108.6 & $\mathrm{C} 11-\mathrm{C} 10-\mathrm{Fe} 3$ & $69.2(15)$ \\
\hline C2-C3-H3A & 108.6 & C9-C10-Fe3 & $68.0(15)$ \\
\hline C4-C3-H3B & 108.6 & C11-C10-H10 & 125.3 \\
\hline C2-C3-H3B & 108.6 & C9-C10-H10 & 125.3 \\
\hline H3A-C3-H3B & 107.6 & Fe3-C10-H10 & 129.1 \\
\hline $\mathrm{O} 3-\mathrm{C} 4-\mathrm{N} 1$ & $120(3)$ & $\mathrm{C} 10-\mathrm{C} 11-\mathrm{C} 12$ & $110(3)$ \\
\hline $\mathrm{O} 3-\mathrm{C} 4-\mathrm{C} 3$ & $123(3)$ & $\mathrm{C} 10-\mathrm{C} 11-\mathrm{Fe} 3$ & $72.0(15)$ \\
\hline N1-C4-C3 & $117(3)$ & $\mathrm{C} 12-\mathrm{C} 11-\mathrm{Fe} 3$ & $70.9(15)$ \\
\hline N1-C5-C6 & $110(2)$ & C10-C11-H11 & 125.1 \\
\hline N1-C5-H5A & 109.6 & C12-C11-H11 & 125.1 \\
\hline C6-C5-H5A & 109.6 & Fe3-C11-H11 & 123.6 \\
\hline N1-C5-H5B & 109.6 & C8-C12-C11 & $106(3)$ \\
\hline C6-C5-H5B & 109.6 & $\mathrm{C} 8-\mathrm{C} 12-\mathrm{Fe} 3$ & $68.7(17)$ \\
\hline H5A-C5-H5B & 108.1 & $\mathrm{C} 11-\mathrm{C} 12-\mathrm{Fe} 3$ & $67.8(15)$ \\
\hline N2-C6-C5 & $113(2)$ & C8-C12-H12 & 127.1 \\
\hline N2-C6-H6A & 108.9 & C11-C12-H12 & 127.1 \\
\hline C5-C6-H6A & 108.9 & Fe3-C12-H12 & 127.9 \\
\hline N2-C6-H6B & 108.9 & C17-C13-C14 & $108(3)$ \\
\hline C5-C6-H6B & 108.9 & $\mathrm{C} 17-\mathrm{C} 13-\mathrm{Fe} 3$ & $69.8(16)$ \\
\hline H6A-C6-H6B & 107.7 & $\mathrm{C} 14-\mathrm{C} 13-\mathrm{Fe} 3$ & $69.6(15)$ \\
\hline $\mathrm{O} 4-\mathrm{C} 7-\mathrm{N} 2$ & $119(2)$ & C17-C13-H13 & 125.9 \\
\hline $\mathrm{O} 4-\mathrm{C} 7-\mathrm{C} 8$ & $123(3)$ & C14-C13-H13 & 125.9 \\
\hline N2-C7-C8 & $117(3)$ & Fe3-C13-H13 & 126.3 \\
\hline $\mathrm{C} 12-\mathrm{C} 8-\mathrm{C} 7$ & $123(3)$ & C15-C14-C13 & $103(3)$ \\
\hline $\mathrm{C} 12-\mathrm{C} 8-\mathrm{C} 9$ & $108(2)$ & $\mathrm{C} 15-\mathrm{C} 14-\mathrm{Fe} 3$ & $66.7(16)$ \\
\hline C7-C8-C9 & $129(3)$ & $\mathrm{C} 13-\mathrm{C} 14-\mathrm{Fe} 3$ & $68.5(16)$ \\
\hline
\end{tabular}




\begin{tabular}{|c|c|c|c|}
\hline C15-C14-H14 & 128.5 & O7-C21-N3 & $124(2)$ \\
\hline C13-C14-H14 & 128.5 & $\mathrm{O} 7-\mathrm{C} 21-\mathrm{C} 20$ & $123(3)$ \\
\hline Fe3-C14-H14 & 127.7 & N3-C21-C20 & $113(3)$ \\
\hline C16-C15-C14 & $112(3)$ & $\mathrm{N} 3-\mathrm{C} 22-\mathrm{C} 23$ & $111(2)$ \\
\hline $\mathrm{C} 16-\mathrm{C} 15-\mathrm{Fe} 3$ & $72.2(18)$ & N3-C22-H22A & 109.5 \\
\hline C14-C15-Fe3 & $71.8(17)$ & $\mathrm{C} 23-\mathrm{C} 22-\mathrm{H} 22 \mathrm{~A}$ & 109.5 \\
\hline C16-C15-H15 & 124.0 & N3-C22-H22B & 109.5 \\
\hline C14-C15-H15 & 124.0 & $\mathrm{C} 23-\mathrm{C} 22-\mathrm{H} 22 \mathrm{~B}$ & 109.5 \\
\hline Fe3-C15-H15 & 123.6 & $\mathrm{H} 22 \mathrm{~A}-\mathrm{C} 22-\mathrm{H} 22 \mathrm{~B}$ & 108.1 \\
\hline C15-C16-C17 & $108(3)$ & $\mathrm{N} 4-\mathrm{C} 23-\mathrm{C} 22$ & $110(2)$ \\
\hline C15-C16-Fe3 & $68.2(18)$ & N4-C23-H23A & 109.7 \\
\hline C17-C16-Fe3 & $70.1(18)$ & $\mathrm{C} 22-\mathrm{C} 23-\mathrm{H} 23 \mathrm{~A}$ & 109.7 \\
\hline C15-C16-H16 & 126.2 & $\mathrm{~N} 4-\mathrm{C} 23-\mathrm{H} 23 \mathrm{~B}$ & 109.7 \\
\hline C17-C16-H16 & 126.2 & $\mathrm{C} 22-\mathrm{C} 23-\mathrm{H} 23 \mathrm{~B}$ & 109.7 \\
\hline Fe3-C16-H16 & 127.1 & $\mathrm{H} 23 \mathrm{~A}-\mathrm{C} 23-\mathrm{H} 23 \mathrm{~B}$ & 108.2 \\
\hline C16-C17-C13 & $109(3)$ & $\mathrm{O} 10-\mathrm{C} 42-\mathrm{O} 12$ & $121(3)$ \\
\hline C16-C17-Fe3 & $69.9(18)$ & $\mathrm{O} 10-\mathrm{C} 42-\mathrm{C} 41$ & $120(3)$ \\
\hline C13-C17-Fe3 & $69.9(17)$ & O12-C42-C41 & $118(3)$ \\
\hline C16-C17-H17 & 125.4 & O11-C41-O9 & $128(4)$ \\
\hline C13-C17-H17 & 125.4 & O11-C41-C42 & $120(3)$ \\
\hline Fe3-C17-H17 & 126.3 & O9-C41-C42 & $112(3)$ \\
\hline O6-C18-O5 & $126(3)$ & O8-C24-N4 & $123(2)$ \\
\hline O6-C18-C19 & $119(3)$ & $\mathrm{O} 8-\mathrm{C} 24-\mathrm{C} 25$ & $119(3)$ \\
\hline O5-C18-C19 & $115(2)$ & N4-C24-C25 & $118(3)$ \\
\hline C18-C19-C20 & $112(2)$ & C29-C25-C26 & $104(3)$ \\
\hline C18-C19-H19A & 109.2 & $\mathrm{C} 29-\mathrm{C} 25-\mathrm{C} 24$ & $130(3)$ \\
\hline C20-C19-H19A & 109.2 & C26-C25-C24 & $126(3)$ \\
\hline C18-C19-H19B & 109.2 & $\mathrm{C} 29-\mathrm{C} 25-\mathrm{Fe} 2$ & $67.0(17)$ \\
\hline C20-C19-H19B & 109.2 & $\mathrm{C} 26-\mathrm{C} 25-\mathrm{Fe} 2$ & $69.8(18)$ \\
\hline H19A-C19-H19B & 107.9 & $\mathrm{C} 24-\mathrm{C} 25-\mathrm{Fe} 2$ & $130(2)$ \\
\hline C21-C20-C19 & $107(2)$ & $\mathrm{C} 25-\mathrm{C} 26-\mathrm{C} 27$ & $114(3)$ \\
\hline $\mathrm{C} 21-\mathrm{C} 20-\mathrm{H} 20 \mathrm{~A}$ & 110.4 & $\mathrm{C} 25-\mathrm{C} 26-\mathrm{Fe} 2$ & $69.9(18)$ \\
\hline C19-C20-H20A & 110.4 & $\mathrm{C} 27-\mathrm{C} 26-\mathrm{Fe} 2$ & $71.7(18)$ \\
\hline C21-C20-H20B & 110.4 & $\mathrm{C} 25-\mathrm{C} 26-\mathrm{H} 26$ & 123.0 \\
\hline C19-C20-H20B & 110.4 & $\mathrm{C} 27-\mathrm{C} 26-\mathrm{H} 26$ & 123.0 \\
\hline H20A-C20-H20B & 108.6 & $\mathrm{Fe} 2-\mathrm{C} 26-\mathrm{H} 26$ & 127.1 \\
\hline
\end{tabular}




\begin{tabular}{|c|c|c|c|}
\hline C26-C27-C28 & $104(3)$ & C34-C33-C32 & $112(3)$ \\
\hline $\mathrm{C} 26-\mathrm{C} 27-\mathrm{Fe} 2$ & $68.1(17)$ & $\mathrm{C} 34-\mathrm{C} 33-\mathrm{Fe} 2$ & $74(2)$ \\
\hline $\mathrm{C} 28-\mathrm{C} 27-\mathrm{Fe} 2$ & $66.4(18)$ & $\mathrm{C} 32-\mathrm{C} 33-\mathrm{Fe} 2$ & $70(2)$ \\
\hline C26-C27-H27 & 128.1 & C34-C33-H33 & 124.0 \\
\hline C28-C27-H27 & 128.1 & C32-C33-H33 & 124.0 \\
\hline $\mathrm{Fe} 2-\mathrm{C} 27-\mathrm{H} 27$ & 128.8 & Fe2-C33-H33 & 123.6 \\
\hline C29-C28-C27 & $108(3)$ & C33-C34-C30 & $99(3)$ \\
\hline $\mathrm{C} 29-\mathrm{C} 28-\mathrm{Fe} 2$ & $67.8(18)$ & $\mathrm{C} 33-\mathrm{C} 34-\mathrm{Fe} 2$ & $66(2)$ \\
\hline $\mathrm{C} 27-\mathrm{C} 28-\mathrm{Fe} 2$ & $72.6(18)$ & $\mathrm{C} 30-\mathrm{C} 34-\mathrm{Fe} 2$ & $66.0(19)$ \\
\hline C29-C28-H28 & 126.2 & C33-C34-H34 & 130.3 \\
\hline C27-C28-H28 & 126.2 & C30-C34-H34 & 130.3 \\
\hline $\mathrm{Fe} 2-\mathrm{C} 28-\mathrm{H} 28$ & 125.1 & $\mathrm{Fe} 2-\mathrm{C} 34-\mathrm{H} 34$ & 128.9 \\
\hline C25-C29-C28 & $111(3)$ & N5-C35-C40 & $117(3)$ \\
\hline $\mathrm{C} 25-\mathrm{C} 29-\mathrm{Fe} 2$ & $72.1(18)$ & N5-C35-C36 & $109(2)$ \\
\hline $\mathrm{C} 28-\mathrm{C} 29-\mathrm{Fe} 2$ & $70.0(18)$ & $\mathrm{C} 40-\mathrm{C} 35-\mathrm{C} 36$ & $109(2)$ \\
\hline $\mathrm{C} 25-\mathrm{C} 29-\mathrm{H} 29$ & 124.6 & N5-C35-H35 & 107.1 \\
\hline C28-C29-H29 & 124.6 & C40-C35-H35 & 107.1 \\
\hline $\mathrm{Fe} 2-\mathrm{C} 29-\mathrm{H} 29$ & 124.8 & C36-C35-H35 & 107.1 \\
\hline C31-C30-C34 & $111(3)$ & N6-C36-C37 & $115(3)$ \\
\hline C31-C30-Fe2 & $70.7(19)$ & N6-C36-C35 & $106(2)$ \\
\hline C34-C30-Fe2 & $71.3(19)$ & C37-C36-C35 & $111(2)$ \\
\hline C31-C30-H30 & 124.6 & N6-C36-H36 & 108.1 \\
\hline $\mathrm{C} 34-\mathrm{C} 30-\mathrm{H} 30$ & 124.6 & C37-C36-H36 & 108.1 \\
\hline Fe2-C30-H30 & 125.0 & C35-C36-H36 & 108.1 \\
\hline C32-C31-C30 & $108(4)$ & C38-C37-C36 & $114(3)$ \\
\hline C32-C31-Fe2 & $70(2)$ & C38-C37-H37A & 108.7 \\
\hline $\mathrm{C} 30-\mathrm{C} 31-\mathrm{Fe} 2$ & $68.2(19)$ & C36-C37-H37A & 108.7 \\
\hline C32-C31-H31 & 126.2 & C38-C37-H37B & 108.7 \\
\hline C30-C31-H31 & 126.2 & C36-C37-H37B & 108.7 \\
\hline $\mathrm{Fe} 2-\mathrm{C} 31-\mathrm{H} 31$ & 126.7 & H37A-C37-H37B & 107.6 \\
\hline C31-C32-C33 & $110(4)$ & C37-C38-C39 & $107(3)$ \\
\hline $\mathrm{C} 31-\mathrm{C} 32-\mathrm{Fe} 2$ & $73(2)$ & C37-C38-H38A & 110.3 \\
\hline $\mathrm{C} 33-\mathrm{C} 32-\mathrm{Fe} 2$ & $68(2)$ & C39-C38-H38A & 110.3 \\
\hline C31-C32-H32 & 124.9 & C37-C38-H38B & 110.3 \\
\hline C33-C32-H32 & 124.9 & C39-C38-H38B & 110.3 \\
\hline Fe2-C32-H32 & 126.2 & H38A-C38-H38B & 108.6 \\
\hline
\end{tabular}




\begin{tabular}{|c|c|c|c|}
\hline C38-C39-C40 & $113(3)$ & $\mathrm{C} 30-\mathrm{Fe} 2-\mathrm{C} 31$ & $41.1(13)$ \\
\hline C38-C39-H39A & 108.9 & $\mathrm{C} 26-\mathrm{Fe} 2-\mathrm{C} 31$ & $109.0(13)$ \\
\hline C40-C39-H39A & 108.9 & $\mathrm{C} 25-\mathrm{Fe} 2-\mathrm{C} 31$ & $124.6(12)$ \\
\hline C38-C39-H39B & 108.9 & $\mathrm{C} 32-\mathrm{Fe} 2-\mathrm{C} 31$ & $37.0(12)$ \\
\hline C40-C39-H39B & 108.9 & $\mathrm{C} 29-\mathrm{Fe} 2-\mathrm{C} 27$ & $69.5(12)$ \\
\hline H39A-C39-H39B & 107.7 & $\mathrm{C} 28-\mathrm{Fe} 2-\mathrm{C} 27$ & 41.1(12) \\
\hline C35-C40-C39 & $110(3)$ & $\mathrm{C} 33-\mathrm{Fe} 2-\mathrm{C} 27$ & $123.9(15)$ \\
\hline $\mathrm{C} 35-\mathrm{C} 40-\mathrm{H} 40 \mathrm{~A}$ & 109.7 & $\mathrm{C} 30-\mathrm{Fe} 2-\mathrm{C} 27$ & $156.0(14)$ \\
\hline C39-C40-H40A & 109.7 & $\mathrm{C} 26-\mathrm{Fe} 2-\mathrm{C} 27$ & $40.2(12)$ \\
\hline C35-C40-H40B & 109.7 & $\mathrm{C} 25-\mathrm{Fe} 2-\mathrm{C} 27$ & $69.9(13)$ \\
\hline C39-C40-H40B & 109.7 & $\mathrm{C} 32-\mathrm{Fe} 2-\mathrm{C} 27$ & $107.0(14)$ \\
\hline $\mathrm{H} 40 \mathrm{~A}-\mathrm{C} 40-\mathrm{H} 40 \mathrm{~B}$ & 108.2 & $\mathrm{C} 31-\mathrm{Fe} 2-\mathrm{C} 27$ & $119.4(13)$ \\
\hline $\mathrm{C} 29-\mathrm{Fe} 2-\mathrm{C} 28$ & $42.3(11)$ & C29-Fe2-C34 & $105.9(14)$ \\
\hline C29-Fe2-C33 & $122.5(14)$ & $\mathrm{C} 28-\mathrm{Fe} 2-\mathrm{C} 34$ & $122.1(15)$ \\
\hline C28-Fe2-C33 & $107.2(15)$ & C33-Fe2-C34 & $40.4(13)$ \\
\hline C29-Fe2-C30 & $125.2(14)$ & C30-Fe2-C34 & $42.8(13)$ \\
\hline $\mathrm{C} 28-\mathrm{Fe} 2-\mathrm{C} 30$ & $162.4(14)$ & $\mathrm{C} 26-\mathrm{Fe} 2-\mathrm{C} 34$ & $158.1(13)$ \\
\hline C33-Fe2-C30 & $67.3(15)$ & $\mathrm{C} 25-\mathrm{Fe} 2-\mathrm{C} 34$ & $120.6(14)$ \\
\hline $\mathrm{C} 29-\mathrm{Fe} 2-\mathrm{C} 26$ & $66.8(12)$ & $\mathrm{C} 32-\mathrm{Fe} 2-\mathrm{C} 34$ & $69.9(15)$ \\
\hline $\mathrm{C} 28-\mathrm{Fe} 2-\mathrm{C} 26$ & $67.5(13)$ & C31-Fe2-C34 & $71.1(14)$ \\
\hline $\mathrm{C} 33-\mathrm{Fe} 2-\mathrm{C} 26$ & $161.0(14)$ & $\mathrm{C} 27-\mathrm{Fe} 2-\mathrm{C} 34$ & $159.3(14)$ \\
\hline $\mathrm{C} 30-\mathrm{Fe} 2-\mathrm{C} 26$ & $123.1(14)$ & $\mathrm{C} 15-\mathrm{Fe} 3-\mathrm{C} 9$ & $119.0(13)$ \\
\hline $\mathrm{C} 29-\mathrm{Fe} 2-\mathrm{C} 25$ & $40.9(11)$ & $\mathrm{C} 15-\mathrm{Fe} 3-\mathrm{C} 11$ & $127.0(11)$ \\
\hline $\mathrm{C} 28-\mathrm{Fe} 2-\mathrm{C} 25$ & $70.5(12)$ & C9-Fe3-C11 & $68.1(12)$ \\
\hline $\mathrm{C} 33-\mathrm{Fe} 2-\mathrm{C} 25$ & $157.3(14)$ & $\mathrm{C} 15-\mathrm{Fe} 3-\mathrm{C} 8$ & $153.7(12)$ \\
\hline $\mathrm{C} 30-\mathrm{Fe} 2-\mathrm{C} 25$ & $107.8(14)$ & $\mathrm{C} 9-\mathrm{Fe} 3-\mathrm{C} 8$ & $41.6(11)$ \\
\hline $\mathrm{C} 26-\mathrm{Fe} 2-\mathrm{C} 25$ & $40.3(11)$ & $\mathrm{C} 11-\mathrm{Fe} 3-\mathrm{C} 8$ & $68.6(11)$ \\
\hline $\mathrm{C} 29-\mathrm{Fe} 2-\mathrm{C} 32$ & $159.7(13)$ & $\mathrm{C} 15-\mathrm{Fe} 3-\mathrm{C} 16$ & $39.6(11)$ \\
\hline C28-Fe2-C32 & $122.1(14)$ & $\mathrm{C} 9-\mathrm{Fe} 3-\mathrm{C} 16$ & $152.6(12)$ \\
\hline C33-Fe2-C32 & $41.8(14)$ & $\mathrm{C} 11-\mathrm{Fe} 3-\mathrm{C} 16$ & $108.8(11)$ \\
\hline C30-Fe2-C32 & $65.9(15)$ & $\mathrm{C} 8-\mathrm{Fe} 3-\mathrm{C} 16$ & $164.8(12)$ \\
\hline $\mathrm{C} 26-\mathrm{Fe} 2-\mathrm{C} 32$ & 124.1(14) & $\mathrm{C} 15-\mathrm{Fe} 3-\mathrm{C} 17$ & $67.2(12)$ \\
\hline $\mathrm{C} 25-\mathrm{Fe} 2-\mathrm{C} 32$ & $158.4(13)$ & C9-Fe3-C17 & $165.5(11)$ \\
\hline C29-Fe2-C31 & 162.1(13) & $\mathrm{C} 11-\mathrm{Fe} 3-\mathrm{C} 17$ & $120.0(12)$ \\
\hline $\mathrm{C} 28-\mathrm{Fe} 2-\mathrm{C} 31$ & $154.2(13)$ & $\mathrm{C} 8-\mathrm{Fe} 3-\mathrm{C} 17$ & $127.3(12)$ \\
\hline C33-Fe2-C31 & $67.2(14)$ & $\mathrm{C} 16-\mathrm{Fe} 3-\mathrm{C} 17$ & $40.0(11)$ \\
\hline
\end{tabular}




\begin{tabular}{|c|c|c|c|}
\hline C15-Fe3-C13 & $68.6(11)$ & $\mathrm{C} 21-\mathrm{N} 3-\mathrm{C} 22$ & $119(2)$ \\
\hline C9-Fe3-C13 & $127.3(12)$ & C21-N3-H3 & 120.5 \\
\hline C11-Fe3-C13 & $153.3(12)$ & C22-N3-H3 & 120.5 \\
\hline $\mathrm{C} 8-\mathrm{Fe} 3-\mathrm{C} 13$ & $107.2(11)$ & C24-N4-C23 & $121(2)$ \\
\hline C16-Fe3-C13 & $68.0(11)$ & C24-N4-H4 & 119.7 \\
\hline $\mathrm{C} 17-\mathrm{Fe} 3-\mathrm{C} 13$ & $40.3(11)$ & C23-N4-H4 & 119.7 \\
\hline C15-Fe3-C14 & $41.6(10)$ & C35-N5-Pt1 & $109.8(19)$ \\
\hline C9-Fe3-C14 & $106.1(12)$ & C35-N5-H5C & 109.7 \\
\hline C11-Fe3-C14 & $164.0(11)$ & Pt1-N5-H5C & 109.7 \\
\hline $\mathrm{C} 8-\mathrm{Fe} 3-\mathrm{C} 14$ & $117.6(11)$ & C35-N5-H5D & 109.7 \\
\hline $\mathrm{C} 16-\mathrm{Fe} 3-\mathrm{C} 14$ & 69.1(11) & Pt1-N5-H5D & 109.7 \\
\hline C17-Fe3-C14 & $69.2(12)$ & H5C-N5-H5D & 108.2 \\
\hline C13-Fe3-C14 & $41.9(11)$ & C36-N6-Pt1 & $108.8(18)$ \\
\hline C15-Fe3-C10 & $109.5(12)$ & C36-N6-H6C & 109.9 \\
\hline C9-Fe3-C10 & $40.6(11)$ & Pt1-N6-H6C & 109.9 \\
\hline C11-Fe3-C10 & $38.8(10)$ & C36-N6-H6D & 109.9 \\
\hline $\mathrm{C} 8-\mathrm{Fe} 3-\mathrm{C} 10$ & $68.1(11)$ & Pt1-N6-H6D & 109.9 \\
\hline C16-Fe3-C10 & $119.9(12)$ & H6C-N6-H6D & 108.3 \\
\hline $\mathrm{C} 17-\mathrm{Fe} 3-\mathrm{C} 10$ & $152.8(12)$ & C1-O1-Pt1 & $124.9(18)$ \\
\hline $\mathrm{C} 13-\mathrm{Fe} 3-\mathrm{C} 10$ & $166.2(12)$ & C18-O5-Pt1 & $125.3(16)$ \\
\hline C14-Fe3-C10 & $127.5(12)$ & C41-O9-Pt1 & $114(2)$ \\
\hline C15-Fe3-C12 & $164.7(11)$ & C42-O10-Pt1 & $109(2)$ \\
\hline $\mathrm{C} 9-\mathrm{Fe} 3-\mathrm{C} 12$ & $69.2(12)$ & O9-Pt1-O1 & $88.5(8)$ \\
\hline C11-Fe3-C12 & 41.4(9) & O9-Pt1-O5 & $92.8(8)$ \\
\hline $\mathrm{C} 8-\mathrm{Fe} 3-\mathrm{C} 12$ & $40.7(11)$ & O1-Pt1-O5 & $178.0(7)$ \\
\hline $\mathrm{C} 16-\mathrm{Fe} 3-\mathrm{C} 12$ & $127.6(11)$ & O9-Pt1-O10 & $84.0(8)$ \\
\hline C17-Fe3-C12 & $108.2(12)$ & O1-Pt1-O10 & $86.0(8)$ \\
\hline $\mathrm{C} 13-\mathrm{Fe} 3-\mathrm{C} 12$ & $118.1(11)$ & O5-Pt1-O10 & $95.7(8)$ \\
\hline C14-Fe3-C12 & $152.3(11)$ & O9-Pt1-N6 & $177.4(10)$ \\
\hline $\mathrm{C} 10-\mathrm{Fe} 3-\mathrm{C} 12$ & $67.5(11)$ & O1-Pt1-N6 & $91.6(9)$ \\
\hline C4-N1-C5 & $124(2)$ & O5-Pt1-N6 & $87.2(8)$ \\
\hline C4-N1-H1 & 117.8 & O10-Pt1-N6 & 93.4(9) \\
\hline C5-N1-H1 & 117.8 & O9-Pt1-N5 & $97.9(10)$ \\
\hline C7-N2-C6 & $122(2)$ & O1-Pt1-N5 & $94.8(9)$ \\
\hline C7-N2-H2 & 118.8 & O5-Pt1-N5 & $83.5(8)$ \\
\hline C6-N2-H2 & 118.8 & O10-Pt1-N5 & $177.9(9)$ \\
\hline
\end{tabular}


N6-Pt1-N5

84.6(9) 
Table S11. Anisotropic displacement parameters $\left(\AA^{2} \times 10^{3}\right)$ for 2 . The anisotropic displacement factor exponent takes the form: $-2 \pi^{2}\left[h^{2} a^{* 2} U^{11}+\ldots+2 h k a^{*} b^{*} U^{12}\right]$

\begin{tabular}{|c|c|c|c|c|c|c|}
\hline & $\mathrm{U}^{11}$ & $\mathrm{U}^{22}$ & $\mathrm{U}^{33}$ & $\mathrm{U}^{23}$ & $\mathrm{U}^{13}$ & $\mathrm{U}^{12}$ \\
\hline $\mathrm{C} 1$ & $36(10)$ & $20(11)$ & $34(9)$ & $-4(8)$ & $10(7)$ & $2(8)$ \\
\hline $\mathrm{C} 2$ & $36(13)$ & $51(13)$ & $40(9)$ & $-13(9)$ & $16(8)$ & $-13(12)$ \\
\hline $\mathrm{C} 3$ & $20(11)$ & $43(10)$ & $37(10)$ & $-8(7)$ & 11(9) & $-3(8)$ \\
\hline $\mathrm{C} 4$ & $18(9)$ & 43(9) & $33(10)$ & $-11(8)$ & $9(8)$ & $-5(7)$ \\
\hline $\mathrm{C} 5$ & $2(10)$ & $38(10)$ & $32(9)$ & $-9(7)$ & $5(7)$ & $2(8)$ \\
\hline C6 & $9(10)$ & $30(10)$ & $31(8)$ & $-6(7)$ & $3(7)$ & $3(8)$ \\
\hline $\mathrm{C} 7$ & $22(8)$ & $28(9)$ & $28(9)$ & $-2(7)$ & $-4(6)$ & $8(6)$ \\
\hline $\mathrm{C} 8$ & $30(9)$ & $33(7)$ & $25(7)$ & $-4(5)$ & $0(6)$ & $-3(6)$ \\
\hline C9 & $32(9)$ & $41(8)$ & $24(7)$ & $-5(6)$ & $-2(6)$ & $-5(6)$ \\
\hline $\mathrm{C} 10$ & $27(9)$ & $40(10)$ & $27(7)$ & $-4(6)$ & $-1(6)$ & $-9(8)$ \\
\hline $\mathrm{C} 11$ & $26(8)$ & $38(10)$ & $26(7)$ & $-10(6)$ & $-2(6)$ & $-9(7)$ \\
\hline $\mathrm{C} 12$ & $28(8)$ & $34(8)$ & $28(8)$ & $-9(6)$ & $-2(6)$ & $-5(6)$ \\
\hline $\mathrm{C} 13$ & $36(10)$ & $41(10)$ & $28(7)$ & $-2(6)$ & $4(6)$ & $-3(8)$ \\
\hline $\mathrm{C} 14$ & $34(9)$ & $46(10)$ & $34(8)$ & $-2(7)$ & $6(6)$ & $-2(7)$ \\
\hline C15 & $36(9)$ & $42(9)$ & $30(9)$ & $-2(7)$ & $5(6)$ & $-8(7)$ \\
\hline $\mathrm{C} 16$ & $39(10)$ & $38(7)$ & $33(8)$ & $-4(6)$ & $9(7)$ & $-5(7)$ \\
\hline $\mathrm{C} 17$ & $40(10)$ & $40(9)$ & $34(8)$ & $-4(6)$ & $3(7)$ & $-1(7)$ \\
\hline $\mathrm{C} 18$ & $70(20)$ & $33(7)$ & $37(8)$ & $2(6)$ & $-3(9)$ & $-7(8)$ \\
\hline C19 & $80(30)$ & $37(10)$ & $34(8)$ & $0(7)$ & $-8(9)$ & 1(12) \\
\hline $\mathrm{C} 20$ & $38(14)$ & $37(10)$ & $36(8)$ & $-2(7)$ & $-1(8)$ & $1(11)$ \\
\hline $\mathrm{C} 21$ & $23(9)$ & $36(12)$ & $32(8)$ & $-2(8)$ & $-9(7)$ & $3(8)$ \\
\hline $\mathrm{C} 22$ & $11(11)$ & $33(11)$ & $34(8)$ & $-1(7)$ & $-4(7)$ & $-2(8)$ \\
\hline $\mathrm{C} 23$ & $9(10)$ & $34(10)$ & $35(10)$ & $-1(7)$ & $-2(8)$ & $-5(8)$ \\
\hline $\mathrm{C} 42$ & $61(10)$ & $19(10)$ & $36(9)$ & $0(8)$ & $-3(7)$ & $0(8)$ \\
\hline C41 & $63(10)$ & $22(11)$ & $40(10)$ & $0(9)$ & $-5(7)$ & $1(8)$ \\
\hline $\mathrm{C} 24$ & $16(7)$ & $33(8)$ & 21(9) & $-6(7)$ & $1(6)$ & $-3(6)$ \\
\hline $\mathrm{C} 25$ & $23(9)$ & $39(7)$ & $38(9)$ & $3(6)$ & $-1(7)$ & $-4(6)$ \\
\hline $\mathrm{C} 26$ & $22(9)$ & $42(9)$ & $38(8)$ & $3(6)$ & $-3(7)$ & $-5(7)$ \\
\hline $\mathrm{C} 27$ & $23(10)$ & $49(10)$ & $51(8)$ & $7(7)$ & $-5(6)$ & $-9(8)$ \\
\hline $\mathrm{C} 28$ & $31(9)$ & $42(10)$ & $57(9)$ & $11(7)$ & $-5(7)$ & $-8(7)$ \\
\hline $\mathrm{C} 29$ & $23(8)$ & $36(8)$ & $51(10)$ & $5(7)$ & $0(6)$ & $-5(6)$ \\
\hline
\end{tabular}




\begin{tabular}{|c|c|c|c|c|c|c|}
\hline $\mathrm{C} 30$ & $54(11)$ & $55(11)$ & $54(8)$ & $-5(7)$ & $-14(7)$ & $-6(9)$ \\
\hline C31 & $38(9)$ & $56(11)$ & $59(10)$ & $-8(8)$ & $-15(7)$ & $-3(8)$ \\
\hline C32 & $35(10)$ & $51(10)$ & $63(11)$ & $-4(8)$ & $-12(8)$ & $-7(7)$ \\
\hline C33 & $42(11)$ & $51(9)$ & $72(11)$ & $-7(8)$ & $-10(8)$ & $-1(8)$ \\
\hline C34 & $49(12)$ & $63(12)$ & $67(9)$ & $-8(8)$ & $-5(8)$ & $-5(9)$ \\
\hline C35 & $50(10)$ & $24(10)$ & $32(9)$ & $3(8)$ & $5(7)$ & $-2(8)$ \\
\hline C36 & $43(10)$ & $29(11)$ & $32(8)$ & $2(7)$ & $4(7)$ & $-5(8)$ \\
\hline C37 & $67(13)$ & $38(14)$ & $41(11)$ & $8(10)$ & $-4(9)$ & $6(10)$ \\
\hline C38 & $80(15)$ & $40(16)$ & $35(10)$ & $7(10)$ & $-1(9)$ & $0(12)$ \\
\hline C39 & $85(15)$ & $41(14)$ & $42(14)$ & $10(11)$ & $-1(10)$ & 1(11) \\
\hline $\mathrm{C} 40$ & $58(13)$ & $38(14)$ & $34(10)$ & $9(10)$ & $8(8)$ & $-6(10)$ \\
\hline $\mathrm{Fe} 2$ & $25(2)$ & $26(3)$ & $48(2)$ & $6(2)$ & $-5(2)$ & $-2(2)$ \\
\hline $\mathrm{Fe} 3$ & $27(2)$ & $31(2)$ & $25(2)$ & $-6(2)$ & $3(2)$ & $-1(2)$ \\
\hline N1 & $17(9)$ & $37(9)$ & $33(9)$ & $-7(7)$ & $8(7)$ & $2(7)$ \\
\hline $\mathrm{N} 2$ & $19(8)$ & $16(9)$ & $21(7)$ & $3(7)$ & $-4(6)$ & $4(7)$ \\
\hline N3 & $22(10)$ & $31(10)$ & $29(8)$ & $3(7)$ & $-8(7)$ & $2(8)$ \\
\hline N4 & $15(8)$ & $40(9)$ & $32(10)$ & $0(8)$ & $-1(7)$ & $-1(7)$ \\
\hline N5 & $35(9)$ & $23(8)$ & $37(8)$ & $4(7)$ & $0(6)$ & $-3(7)$ \\
\hline N6 & $35(8)$ & $24(9)$ & $32(8)$ & $2(6)$ & $4(6)$ & $-3(7)$ \\
\hline $\mathrm{O} 1$ & $34(8)$ & $21(7)$ & $34(7)$ & $-2(5)$ & $6(6)$ & $0(7)$ \\
\hline $\mathrm{O} 2$ & $51(11)$ & $100(20)$ & $40(10)$ & $-32(12)$ & $18(8)$ & $-30(11)$ \\
\hline $\mathrm{O} 3$ & $21(8)$ & $50(12)$ & $60(13)$ & $0(10)$ & $11(8)$ & $-3(8)$ \\
\hline $\mathrm{O} 4$ & $22(7)$ & $35(10)$ & $36(8)$ & $-5(8)$ & $-4(6)$ & $4(7)$ \\
\hline O5 & $3(7)$ & $28(6)$ & $32(6)$ & $-2(5)$ & $-3(6)$ & $-4(6)$ \\
\hline O6 & $130(20)$ & $40(9)$ & $33(8)$ & $6(7)$ & $1(10)$ & $-7(12)$ \\
\hline $\mathrm{O} 7$ & $27(9)$ & $46(12)$ & $41(10)$ & $-4(9)$ & $-3(7)$ & $6(8)$ \\
\hline $\mathrm{O} 8$ & $15(7)$ & $42(10)$ & $32(9)$ & $5(8)$ & $-1(6)$ & $-2(7)$ \\
\hline O9 & $53(9)$ & $24(8)$ & $41(8)$ & $1(6)$ & $-6(6)$ & $-1(7)$ \\
\hline $\mathrm{O} 10$ & $55(9)$ & $27(8)$ & $33(7)$ & $5(6)$ & $-1(6)$ & $-7(7)$ \\
\hline O11 & $80(14)$ & $41(11)$ & $56(11)$ & $15(11)$ & $-5(10)$ & $13(11)$ \\
\hline $\mathrm{O} 12$ & $74(13)$ & $36(11)$ & $38(9)$ & $0(8)$ & $-4(8)$ & $-17(10)$ \\
\hline $\mathrm{O} 13$ & $49(18)$ & $61(16)$ & $110(20)$ & $0(14)$ & $4(16)$ & $12(14)$ \\
\hline $\mathrm{O} 14$ & $90(20)$ & $73(18)$ & $51(14)$ & $3(13)$ & $-2(14)$ & $-28(16)$ \\
\hline O15 & $44(18)$ & $64(17)$ & $150(30)$ & $-2(17)$ & $30(19)$ & $-12(16)$ \\
\hline Pt1 & $56(1)$ & $27(1)$ & $28(1)$ & $-1(1)$ & $3(1)$ & $-1(1)$ \\
\hline
\end{tabular}


Table S12. Hydrogen coordinates ( $\left.x 1^{4}\right)$ and isotropic displacement parameters $\left(\AA^{2} \times 10^{3}\right)$

For 2.

\begin{tabular}{|c|c|c|c|c|}
\hline & $\mathrm{x}$ & $\mathrm{y}$ & z & $\mathrm{U}(\mathrm{eq})$ \\
\hline $\mathrm{H} 2 \mathrm{~A}$ & 5275 & 3789 & 4137 & 51 \\
\hline $\mathrm{H} 2 \mathrm{~B}$ & 6309 & 2778 & 4273 & 51 \\
\hline $\mathrm{H} 3 \mathrm{~A}$ & 8178 & 4218 & 4146 & 40 \\
\hline H3B & 7454 & 3596 & 3856 & 40 \\
\hline $\mathrm{H} 5 \mathrm{~A}$ & 8121 & 7964 & 3795 & 29 \\
\hline H5B & 7201 & 7323 & 3535 & 29 \\
\hline H6A & 9212 & 8199 & 3329 & 28 \\
\hline H6B & 10102 & 7286 & 3538 & 28 \\
\hline H9 & 8098 & 5109 & 2658 & 39 \\
\hline H10 & 8726 & 3379 & 2289 & 38 \\
\hline H11 & 11044 & 2647 & 2408 & 36 \\
\hline H12 & 12132 & 4112 & 2805 & 36 \\
\hline H13 & 9618 & 3598 & 3517 & 42 \\
\hline H14 & 7211 & 3490 & 3254 & 46 \\
\hline H15 & 7408 & 1690 & 2869 & 43 \\
\hline H16 & 9666 & 688 & 2910 & 44 \\
\hline H17 & 11057 & 1868 & 3298 & 46 \\
\hline H19A & 6185 & 9876 & 5521 & 60 \\
\hline H19B & 7770 & 10234 & 5522 & 60 \\
\hline $\mathrm{H} 20 \mathrm{~A}$ & 6710 & 8031 & 5828 & 44 \\
\hline H20B & 8281 & 8458 & 5839 & 44 \\
\hline $\mathrm{H} 22 \mathrm{~A}$ & 7130 & 10704 & 6618 & 31 \\
\hline H22B & 7935 & 11758 & 6432 & 31 \\
\hline $\mathrm{H} 23 \mathrm{~A}$ & 10046 & 10892 & 6614 & 31 \\
\hline $\mathrm{H} 23 \mathrm{~B}$ & 9119 & 11417 & 6888 & 31 \\
\hline $\mathrm{H} 26$ & 8370 & 7879 & 7326 & 41 \\
\hline H27 & 8837 & 5815 & 7579 & 49 \\
\hline $\mathrm{H} 28$ & 11228 & 5159 & 7389 & 52 \\
\hline H29 & 12106 & 6869 & 7041 & 44 \\
\hline H30 & 8910 & 7070 & 6413 & 65 \\
\hline
\end{tabular}




\begin{tabular}{lrlll} 
H31 & 6960 & 6249 & 6739 & 61 \\
H32 & 7656 & 4296 & 6961 & 60 \\
H33 & 10068 & 3792 & 6801 & 66 \\
H34 & 10977 & 5459 & 6430 & 72 \\
H35 & 6627 & 3755 & 5258 & 43 \\
H36 & 7886 & 5653 & 5632 & 42 \\
H37A & 8886 & 3211 & 5518 & 59 \\
H37B & 9667 & 4244 & 5714 & 59 \\
H38A & 7982 & 4154 & 6092 & 62 \\
H38B & 8607 & 2793 & 6036 & 62 \\
H39A & 6180 & 2704 & 6032 & 67 \\
H39B & 6780 & 2283 & 5707 & 67 \\
H40A & 4890 & 3635 & 5626 & 52 \\
H40B & 5605 & 4697 & 5828 & 52 \\
H1 & 8956 & 5812 & 3855 & 35 \\
H2 & 8387 & 6159 & 3118 & 23 \\
H3 & 8953 & 9789 & 6186 & 33 \\
H4 & 8390 & 9165 & 6902 & 35 \\
H5C & 5449 & 6073 & 5370 & 38 \\
H5D & 5106 & 5105 & 5101 & 38 \\
H6C & 8986 & 4652 & 5081 & 36 \\
H6D & 9424 & 5918 & 5249 & 36 \\
\hline & & & & \\
\hline
\end{tabular}




\section{References:}

(S1) a) Kizu, R.; Nakanishi, T.; Hayakawa, K.; Matsuzawa, A.; Eriguchi, M.; Takeda, Y.; Akiyama, N.; Tashiro, T.; Kidani, Y. A new orally active antitumor 1R,2R-cyclohexanediamine-platinum(IV) complex: trans-(n-valerato)chloro(1R,2R-cyclohexanediamine) (oxalato)platinum(IV). Cancer Chemother. Pharmacol. 1999, 43, 97-105; b) Reithofer, M. R.; Valiahdi, S. M.; Jakupec, M. A.; Arion, V. B.; Egger, A.; Galanski, M.; Keppler, B. K. Novel Di- and Tetracarboxylatoplatinum(IV) Complexes. Synthesis, Characterization, Cytotoxic Activity, and DNA Platination. J. Med. Chem. 2007, 50, 6692-6699.

(S2) Banfić, J.; Legin, A. A.; Jakupec, M. A.; Galanski, M.; Keppler, B. K. Platinum(IV) complexes featuring one or two axial ferrocene bearing ligands-synthesis, characterization, and cytotoxicity. Eur. J. Inorg. Chem., 2014, 484-492.

(S3) Hagen, H; Marzenell, P.; Jentzsch, E.; Wenz, F.; Veldwijk, M. R.; Mokhir, A. Aminoferrocenebased prodrugs activated by reactive oxygen species. J. Med. Chem. 2012, 55, 924-934.

(S4) Lukinavičius, G.; Umezawa, K.; Olivier, N.; Honigmann, A.; Yang, G.; Plass, T.; Mueller, V.; Reymond, L.; Corrêa Jr, I. R.; Luo, Z-G.; Schultz, C.; Lemke, E. A.; Heppenstall, P.; Eggeling, C.; Manley, S.; Johnsson, K. A near-infrared fluorophore for live-cell super-resolution microscopy of cellular proteins. Nat. Chem. 2013, 5, 132-139.

(S5) CrysAlisPro. Agilent Technologies. Agilent Technologies UK Ltd., Oxford, UK, SuperNova CCD System, CrysAlicPro Software System, 1.171.37.31. 2013.

(S6) Sheldrick, G. M. SHELXT-Integrated space-group and crystal-structure determination. Acta Cryst. 2015, A71, 3-8.

(S7) Sheldrick, G. M. SHELXL-2014/7. Program for the Refinement of Crystal Structures. 2015, Acta Cryst., C71, 9-18.

(S8) Spek, A. L. PLATON, A Multipurpose Crystallographic Tool. Utrecht University, The Netherlands. 1998.

(S9) Farrugia, L. J. WinGX 1.64. An Integrated System of Windows Programs for the Solution, Refinement and Analysis of Single Crystal X-ray Diffraction Data. J. Appl. Cryst. 1999, 32. 837-838.

(S10) Flack, H. D. On enantiomorph-polarity estimation. Acta Cryst. 1983, A39, 876-881.

(S11) Hooft, R. W. W.; Straver, L. H. and Spek, A. L. Determination of absolute structure using Bayesian statistics on Bijvoet differences. J. Appl. Cryst. 2008, 41, 96-103.

(S12) $\mathrm{R}_{\mathrm{W}}\left(\mathrm{F}^{2}\right)=\left\{\Sigma \mathrm{w}\left(\left|\mathrm{F}_{\mathrm{O}}\right|^{2}-\left|\mathrm{F}_{\mathrm{C}}\right|^{2}\right)^{2} / \Sigma \mathrm{w}\left(\left|\mathrm{F}_{\mathrm{O}}\right|\right)^{4}\right\}^{1 / 2}$ where $\mathrm{w}$ is the weight given each reflection.

$\left.\mathrm{R}(\mathrm{F})=\Sigma\left(\left|\mathrm{F}_{\mathrm{O}}\right|-\left|\mathrm{F}_{\mathrm{C}}\right|\right) / \Sigma\left|\mathrm{F}_{\mathrm{O}}\right|\right\}$ for reflections with $\mathrm{F}_{\mathrm{O}}>4\left(\sigma\left(\mathrm{F}_{\mathrm{O}}\right)\right)$.

$\mathrm{S}=\left[\Sigma \mathrm{w}\left(\left|\mathrm{F}_{\mathrm{O}}\right|^{2}-\left|\mathrm{F}_{\mathrm{C}}\right|^{2}\right)^{2} /(\mathrm{n}-\mathrm{p})\right]^{1 / 2}$, where $\mathrm{n}$ is the number of reflections and $\mathrm{p}$ is the number of refined parameters.

(S13) International Tables for X-ray Crystallography (1992). Vol. C, Tables 4.2.6.8 and

6.1.1.4, A. J. C. Wilson, editor, Boston: Kluwer Academic Press.

(S14) Sheldrick, G. M. SHELXTL/PC (Version 5.03). Siemens Analytical X-ray Instruments, Inc., Madison, Wisconsin, USA. 1994.

(S15) Palatinus, L.; Chapuis, G. SuperFlip-a computer program for the solution of crystal structures by charge flipping in arbitrary dimensions. J. Appl. Cryst. 2007, 40, 786-790.

(S16) Sheldrick, G. M. A short history of SHELX. Acta Cryst., 2008, A64, 112-122. 